\title{
Development, implementation and evaluation of a weight gain prevention programme
}

Citation for published version (APA):

Kwak, L. N. (2007). Development, implementation and evaluation of a weight gain prevention programme. [Doctoral Thesis, Maastricht University]. Universiteit Maastricht. https://doi.org/10.26481/dis.20071214lk

Document status and date:

Published: 01/01/2007

DOI:

10.26481/dis.20071214lk

Document Version:

Publisher's PDF, also known as Version of record

\section{Please check the document version of this publication:}

- A submitted manuscript is the version of the article upon submission and before peer-review. There can be important differences between the submitted version and the official published version of record.

People interested in the research are advised to contact the author for the final version of the publication, or visit the DOI to the publisher's website.

- The final author version and the galley proof are versions of the publication after peer review.

- The final published version features the final layout of the paper including the volume, issue and page numbers.

Link to publication

\footnotetext{
General rights rights.

- You may freely distribute the URL identifying the publication in the public portal. please follow below link for the End User Agreement:

www.umlib.nl/taverne-license

Take down policy

If you believe that this document breaches copyright please contact us at:

repository@maastrichtuniversity.nl

providing details and we will investigate your claim.
}

Copyright and moral rights for the publications made accessible in the public portal are retained by the authors and/or other copyright owners and it is a condition of accessing publications that users recognise and abide by the legal requirements associated with these

- Users may download and print one copy of any publication from the public portal for the purpose of private study or research.

- You may not further distribute the material or use it for any profit-making activity or commercial gain

If the publication is distributed under the terms of Article $25 \mathrm{fa}$ of the Dutch Copyright Act, indicated by the "Taverne" license above, 
THE NHF-NRG

IN BALANCE-PROJECT

LYDIA NADINE KWAK 



\title{
Development, implementation and evaluation of a weight gain prevention programme
}

\author{
PROEFSCHRIFT
}

ter verkrijging van de graad van doctor aan de Universiteit Maastricht, op gezag van de Rector Magnificus, Prof. mr. G.P.M.F. Mols, volgens het besluit van het College van Decanen, in het openbaar te verdedigen op vrijdag 14 december 2007 om 14.00 uur

door

Lydia Nadine Kwak 


\section{Promotores:}

Prof. dr. ir. J. Brug (Vrije Universiteit Medisch Centrum, Amsterdam)

Prof. dr. M.A. van Baak

\section{Copromotor:}

Dr. S.P.J. Kremers

Beoordelingscommissie:

Prof. dr. N.K de Vries (voorzitter)

Prof. dr. E.E. Blaak

Prof. dr. W. van Mechelen (Vrije Universiteit Medisch Centrum, Amsterdam)

Prof. dr. ir. W.H. Saris

Dr. M. Sjöström (Karolinska Institute, Sweden)

Design by NH Vormgevers, Zaandam

Printed by Kwak \& Van Daalen \& Ronday, Zaandam

ISBN: 978-90-71486-02-9

The studies presented in this thesis were performed within the Nutrition and Toxicology Research Institute Maastricht (NUTRIM), which participates in the Graduate School VLAG (Food Technology, Agrobiotechnology, Nutrition and Health Sciences), accredited by the Royal Netherlands Academy of Arts and Sciences.

The study described in this thesis was supported by a grant from the Netherlands Heart Foundation (NHF-2000T204).

Financial support by the Netherlands Heart Foundation for the publication of this thesis is gratefully acknowledged. 
Lang voordat ik

mijn eigen stem

begreep, stelde ik

een eerste vraag.

Nog vaag en onbewust

werd die prille kreet

begraven, om

met mijn durf

te groeien.

Letter voor letter

vormden hun antwoorden

mijn zoektocht, naar

de ware bedoeling.

En,

met elke kleine stap

raakte ik tussen de regels

bevangen door hun waarheid.

Elk verhaal een deel van $\mathrm{mij}$,

geroepen tot de kern

van mijn onstaan.

Ruben Kwak, 2007 



\section{CONTENTS}

$\begin{array}{ll}\text { GENERAL INTRODUCTION } & 8\end{array}$

$\begin{array}{ll}\text { CHAPTER } 2 & 26\end{array}$

Participation rates in worksite-based intervention studies:

health promotion context as a crucial quality criterion

CHAPTER 3

Measuring physical activity in field studies: comparison of a questionnaire, $24 \mathrm{~h}$ recall and accelerometer

CHAPTER 4

Formation of implementation intentions promotes stair use

CHAPTER 5

Long-term effectiveness of implementation intentions regarding stair use: a 12 months follow-up

CHAPTER 6

A poster-based intervention to promote stair use in blue-and white-collar worksites

\section{CHAPTER 7}

The effectiveness of the NHF-NRG In Balance-project: short-and long-term changes in sum of skinfolds and waist circumference

CHAPTER 8

Behavioural and cognitive effects of a worksite-based weight gain prevention programme: The NHF-NRG In Balance-project

GENERAL DISCUSSION

REFERENCES

DANKWOORD 


\section{GENERAL INTRODUCTION}

Based on: Kwak, L., Kremers, S.P.J., Werkman, A., Visscher, T.L.S., Van Baak, M.A. \& Brug, J. (2007) The NHF-NRG In Balance-project: the application of Intervention Mapping in the development, implementation and evaluation of weight gain prevention at the worksite. Obesity Reviews, 8, 347-61 


\section{INTRODUCTION}

The prevalence of overweight and obesity has increased dramatically over the past two decades (Hill et al., 2003). As obesity is associated with many chronic conditions including diabetes, hypertension, hypercholesterolemia, stroke, heart disease, certain cancers, and arthritis, it has become one of the most pressing health concerns (Flegal et al., 2002; Kim \& Popkin, 2006). Many previous attempts to tackle obesity have focused mainly on weight loss. Even though these treatment programmes are often successful on the short-term, long-term effects are limited (Glenny et al., 1997; Klem et al., 2000; Hitchcock \& Pugh, 2002). Emphasis is therefore now being placed on weight gain prevention as a potentially more effective strategy for addressing the overweight and obesity epidemic (Carraro \& Garcia Cebrian, 2003; Davey, 2004; Mullis et al., 2004). To date however, relatively little information is available on the prevention of weight gain (Jeffery, 2001; Caterson \& Gill, 2002; Jakicic \& Otto, 2005). A systematic review of obesity prevention interventions identified only four interventions addressing this issue in adults (Hardeman et al., 2000). The evidence from these interventions and those reported in similar reviews (Douketis et al., 1999; McLean et al., 2003; Mulvihill \& Quigley, 2003) is mixed and inconclusive in terms of effectiveness. Due to the variability of study designs, samples and outcome variables, it is often difficult to identify effective interventions. Notably, studies published to date have provided little detail on interventions, underlying theoretical models and study methods. Unless these models and methods of behaviour change are identified and described more accurately, it will be difficult to conclude which interventions are effective and to replicate effective interventions in different situations (Hardeman et al., 2000). With this paper we hope to contribute to the current dearth of detailed information in this field.

The present paper aims at systematically describing the development, implementation and evaluation framework of the NHF-NRG In Balance-project, a weight gain prevention programme directed at young adults at the worksite. The In Balance-project is part of a larger, multidisciplinary, research programme 'Netherlands Research programme weight Gain prevention' (NRG) and was initiated by the Netherlands Heart Foundation (NHF) (Kremers et al., 2005a). In short, the overall goal of NHF-NRG was to prevent weight gain through changes in both physical activity and food intake. The programme included interventions that were individually and environmentally orientated. This paper will address the 1-year intervention project targeted at adults aged 20-40 years. In total twelve worksites were involved, these included blue-collar companies (e.g. paper factory and furniture manufacture), hospitals, local governments, a university, a water-supplying company and an energy-supplying company. The main inclusion-criteria for participation were that participating companies should have a minimum of 100 employees and provide canteen facilities. Half of the recruited companies received the programme, which included interventions that were individually and environmentally orientated and directed at changes in physical activity and food intake.

The outline of the paper follows the steps as classified in the Intervention Mapping (IM) protocol (figure 1). IM explicates a series of five steps for the development of a health promotion programme based on a foundation of theoretical, empirical and practical information. Each step comprises of different tasks, which results in a clear end product, providing the foundation for the next step. As a result of the problem-driven perspective of IM, different theories might be applied in each step. In this context it is the challenge to find the best theory or combination of theoretical constructs to understand or solve the problem at hand (Bartholomew et al., 2001) $)^{\mathrm{a}}$. The product of IM is an Intervention Map, which can be regarded as a 
blueprint for the design, implementation, and evaluation of the programme. It does not just serve as a way to map the path of intervention development and evaluation, but also provides a framework for documenting decisions. This specificity has been argued to contribute to a more effective programme and enhanced knowledge on the effectiveness of the programme (Bartholomew et al., 2001).

Figure 1. Intervention Mapping Protocol (Bartholomew et al., 2001).

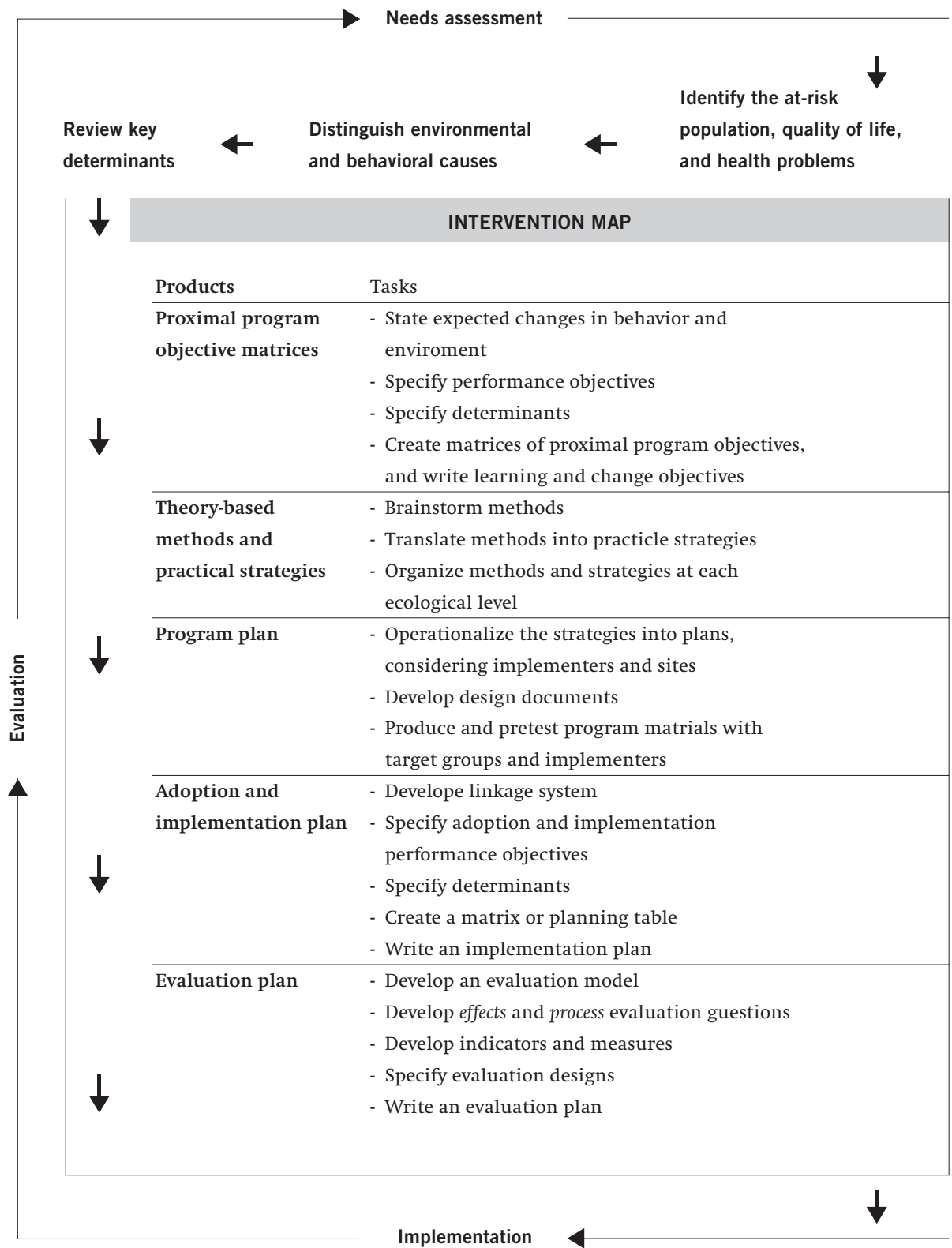

Note. ${ }^{a}$ The development and implementation of the NHF-NRG In Balance-project occured before publication of Bartholomew et al., 2006. 
By systematically describing the development, implementation and evaluation framework of the NHF-NRG In Balance-project this paper has not only taken the form of a guide to systematically develop weight gain prevention interventions, it also gives an overview of the current theoretical and empirical knowledge-base in the field of obesity prevention.

\section{NEEDS ASSESSMENT}

A needs assessment, i.e. a systematic inventory of important health issues and their underlying risk behaviours, is the basis for IM. This needs assessment eventually leads to the specification of the objectives of the intervention programme (Bartholomew et al., 2001). The results of the needs assessment literature review are described below.

It has been well documented that overweight and obesity are major determinants of population health (Flegal et al., 2002; Kim \& Popkin, 2006). Epidemiological data indicate that young adults in the age range of 20-34 years are at particularly high risk of weight gain that may eventually lead to being overweight (Sherwood et al., 2000; Sheehan et al., 2003), as young adulthood is a time period during which important life-events, such as entering the workforce, getting married and/ or having children, may lead to significant changes in lifestyle and subsequently in body weight (Williamson et al., 1990; Prentice \& Jebb, 1995; Burke et al., 1996; Klem et al., 2000; Rothacker \& Blackburn, 2000, Jakicic \& Otto, 2005; Wammes et al., 2005). Data from the Coronary Artery Risk development in Young Adults (CARDIA) study (Lewis et al., 2000) indicated that in the United States the overall weight gain during young to middle adulthood averages $0.70 \mathrm{~kg} / \mathrm{year}$. Dutch data show similar increases, and estimated that the annual weight gain in Dutch young adults is approximately $0.60 \mathrm{~kg} /$ year (Visscher et al., 2002). Longitudinal data from another Dutch study also shows that weight gain is higher in young adults than in other adult categories (Nooyens, A. unpublished data). According to Hill and colleagues (2003) this weight gain corresponds with an energy excess of approximately $100 \mathrm{kcal} /$ day (Hill et al., 2003). Several behavioural risk factors, which are either related to a decrease in energy expenditure and/or an increase in energy intake, may contribute to this energy imbalance (Jakicic, 2002; Brown \& Trost, 2003; Wammes et al., 2005). The decrease in energy expenditure is mainly influenced by the decline in daily routine physical activities (Prentice \& Jebb, 1995; Stephenson et al., 2000), such as walking and cycling as part of regular travel behaviour (Wen et al., 2005) and physical activity at work (Prentice \& Jebb, 1995; Kumanyika et al., 2002). The increase in energy intake can partly be explained by the increased portion sizes of food (Kral \& Rolls, 2004; Rolls et al., 2004; Ello-Martin et al., 2005; Ledikwe et al., 2005) and the increased consumption of high energy-dense foods (Rolls, 2000; Rolls \& Bell, 2000; Ello-Martin et al., 2005; Rolls et al., 2005).

There are several environmental factors that are related to weight gain (Egger \& Swinburn, 1997; Hill \& Peters, 1998; Swinburn et al., 1999; Kumanyika, 2001). An important environmental context of adults in this age group is the worksite, as this is where they spend a large part of their day (Dishman et al., 1998). Moreover, worksites can be identified as ideal settings for weight gain prevention programmes as they provide many opportunities to reinforce the adoption and maintenance of healthy lifestyle behaviours (Kremers et al., 2005a).

The needs assessment resulted in the following programme objective: to decrease overall dietary energy intake and/or increase daily routine physical activity in order to prevent weight gain in young adults aged 25-40 years. 


\section{INTERVENTION MAPPING}

\section{Step 1: Defining proximal programme objectives}

Behaviour change and performance objectives

The first task in step 1 of IM is to translate the previously described overview of risk behaviours into health-promoting behaviours. This has lead to the specification of the main energy balance-related behaviours as depicted in table 1 . The behaviours will be shortly commented here. Energy balance-related behaviours at both sides of the energy balance were identified. Two energy expenditure behaviours were prioritized. The first is increasing the frequency and duration of walking and cycling for transport, this way encouraging the integration of physical activity into daily routine (Kumanyika et al., 2002; Jakicic \& Otto, 2005). The second is increasing the physical activity level at work. It has been suggested that the cumulative effects of small but consistent increases in energy expenditure, such as for example walking to co-workers to interact instead of sending those co-workers e-mails, parking a little further from a destination, conducting a walking meeting and walking up and down flights of stairs every day, could be significant for weight maintenance over longer periods of time (French et al., 2001; Hill et al., 2003).

Table 1. Energy balance-related behaviours.

1. Young adults increase their frequency and duration of walking and cycling for transport;

2. Young adults increase their physical activity level at work;

3. Young adults decrease their portion sizes;

4. Young adults decrease their energy density during the day:

4a. Young adults replace high-fat products by low energy dense products;

4b. Young adults replace products low in fibre by fibre-rich products (e.g. whole-grain products, fruit and vegetables);

4c. Young adults replace saturated fats by unsaturated fats.

Three energy intake behaviours were prioritized. Decreasing portion sizes is the first, since a simple way for individuals to reduce their energy intake without changing the types of food they eat, is simply eating less of the preferred foods. For example, a $100 \mathrm{kcal}$ reduction in intake can be obtained by eating 15\% less of a typical fast-food hamburger (Hill et al., 2003). A reduction in intake of high-fat foods and an increase in high-fibre foods are the second and third health promoting energy intake behaviours, both in order to reduce intake of energy dense foods. Several studies have shown that a decrease in energy intake can be produced by a reduction in dietary fat (Astrup et al., 2000a; Astrup et al., 2000b; Rolls, 2000; Astrup et al., 2002). Increasing dietary fibre, thereby decreasing energy density, may help reduce energy intake by decreasing overall energy density (Howarth et al., 2001; Yao \& Roberts, 2001; Howarth et al., 2005; Slavin, 2005). Several previous intervention studies suggest that combining a high-fibre and low-fat diet may be even more effective in maintaining normal weight than either one alone (Raben et al., 1995; Yao \& Roberts, 2001; Roberts et al., 2002; Howarth et al., 2005). Because existing dietary guidelines recommend replacing saturated fats by unsaturated fats in order to improve cardiovascular health risks, we included this as an additional behaviour change target in our programme (WHO, 2004). 
Next, each identified health promoting behaviour was specified into so-called performance objectives that the target group (or environmental agents) needed to achieve as a result of the NHF-NRG In Balance-project (Bartholomew et al., 2001). Each step in IM involves the use of theories that are most applicable (see table 2). With regard to the specification of performance objectives (step 1) these are self-regulation theory (Boekaerts et al., 2000) and implementation intention theory (Gollwitzer, 1999).

Table 2. Theories used in IM.

\begin{tabular}{|c|c|}
\hline Methods & Theories \\
\hline \multirow{2}{*}{$\begin{array}{l}\text { Specification of performance } \\
\text { objectives }\end{array}$} & Self-regulation theory (e.g., Boekaerts et al., 2000) \\
\hline & Implementation intention theory (Gollwitzer, 1999) \\
\hline \multicolumn{2}{|c|}{ Step 2: Selecting theory-based intervention methods } \\
\hline Methods & Theories \\
\hline $\begin{array}{l}\text { (Self) monitoring } \\
\text { (Self)-evaluation } \\
\text { Goal-setting }\end{array}$ & Self-regulation theory (e.g., Boekaerts et al., 2000) \\
\hline Personalization of risk & $\begin{array}{l}\text { Precaution Adoption Process Model } \\
\text { (unrealistic optimism) (Weinstein, 1988) }\end{array}$ \\
\hline Providing information & Elaboration Likelihood Model (Petty \& Cacioppo, 1986) \\
\hline Tailoring & $\begin{array}{l}\text { Theory of Planned Behaviour (Ajzen, 1991) } \\
\text { Precaution Adoption Process Model (Weinstein, 1988) }\end{array}$ \\
\hline Decisional balance & Transtheoretical Model (Prochaska \& DiClemente, 1983) \\
\hline Skills training & Social Cognitive Theory (Bandura, 1986) \\
\hline Prompting & Habit Theory (e.g., Triandis, 1977) \\
\hline Rewarding & Operant Theory (Skinner, 1963) \\
\hline Environmental change & Social Ecological Theory (e.g., Stokols, 1992) \\
\hline Mobilizing social support & Social Support Theory (Heaney \& Israel, 1997) \\
\hline \multicolumn{2}{|l|}{ Step 5: Monitoring and evaluation plan } \\
\hline Methods & Theories \\
\hline Intermediate indicators of success & $\begin{array}{l}\text { Theory of Planned Behaviour (Ajzen, 1991) } \\
\text { Precaution Adoption Process Model (Weinstein, 1988) } \\
\text { Environmental Research framework for weight Gain } \\
\text { prevention (Kremers et al., 2006) }\end{array}$ \\
\hline Indirect indicators of success & Diffusion of Innovations Model (Rogers, 1995) \\
\hline
\end{tabular}

Self-regulation can be seen as a dynamic motivational system of setting goals, developing and enacting strategies to achieve these goals, appraising progress, and revising goals and strategies accordingly (De Ridder \& De Wit, 2006). Implement-ation intentions can facilitate effective self-regulation of goal striving (Gollwitzer \& Sheeran, 2006), as implementation intentions help people to deal with self-regulatory problems (Sheeran et al., 2006). For the health promoting-behaviour replacing products low in fibre by fibre-rich products, for example, this meant the specification of the individual and environmental performance objectives depicted in table 3. 
Table 3. Example of individual performance objectives related to replacing products low in fibre by fibre-rich products.

Performance objectives for individual changes related to programme objective $4 \mathrm{~b}$ :

1. Young adults know what fibre-rich products are;

2. Young adults self-asses their fibre intake;

3. Young adults indicate reasons for consuming products low in fibre;

4. Young adults have a negative attitude toward products low in fibre;

5. Young adults indicate the importance of consuming fibre-rich products;

6. Young adults propose solutions, i.e. set goals to consume fibre-rich products;

7. Young adults choose to consume fibre-rich products and set reachable goals for themselves;

8. Young adults form implementation intentions specifying the consumption of fibre-rich products;

9. Young adults implement these solutions, thus consuming fibre-rich products;

10. Young adults evaluate their personal goal performance, i.e. consuming fibre-rich products.

Performance objectives for organizational environmental changes related to programme objective $4 \mathrm{~b}$ :

1. The management of the worksite has a positive attitude towards fibre-rich products;

2. The management of the worksite provides the opportunity to change the fibre content of canteen products;

3. The canteen-personnel have a positive attitude towards changing the fibre content of canteen products;

4. The canteen-personnel offer fibre-rich products.

Changeable personal and external determinants

The third task in IM step 1 is to select important and changeable personal and environmental determinants of the performance objectives (Bartholomew et al., 2001). For the present study, determinants were selected based on literature review and focus groups interviews. The following personal determinants were identified: knowledge, awareness, taste preference, attitude, perceived self-efficacy, and habit. The most important environmental determinants that were identified in the literature review for this particular risk group were availability, management commitment, management support, social support from co-workers, company policies and cost. Reviewed determinants mostly originate from cross-sectional studies, as cohort-studies are scarce in this field (Brug et al., 2006). The determinants are described in more detail below.

Firstly, knowledge of the energy balance-related behaviours is of importance. Young adults should, for example, know what constitutes energy balance (Wammes et al., 2005), have nutritional knowledge (Wardle et al., 2000), be familiar with fibre-rich products, know what constitutes 'fibre-rich' (Smith et al., 2003) and know the health benefits of the different identified energy balancerelated behaviours, such as the benefits of eating whole-grain products (Adams \& Engstrom, 2000; Smith et al., 2003) and taking the stairs instead of the elevator (Andersen et al., 1998). Second, individuals' awareness of their own energy balancerelated behaviours is crucial (Van Assema et al., 1996; Lechner et al., 1997; O’Brien 
et al., 2000; Lingwood, 2004; Wammes et al., 2005). Individuals that are for example not accurately aware of their own fruit and vegetable consumption are less likely to change their behaviour (Lechner et al., 1997).

Thirdly, several studies identified taste preferences as important determinants of fat, fibre and fibre-rich food (e.g. fruits and vegetables) consumption (e.g. see also Brug et al., 1995; Krebs-Smith et al., 1995; Hagdrup et al., 1998; Baranowski et al., 1999; Povey et al., 2000; Ma et al., 2002). Fourth, changing existing negative attitudes and reinforcing existing positive attitudes regarding using active transport for travelling (Proper et al., 2000) and being more physically active at work have been identified as being of great importance for improving active transport and being physically active at work (Urlings et al., 1997; Proper et al., 1999; Trost et al., 2002).

In addition, perceived self-efficacy has proved to be an important predictor of the identified energy balance-related behaviours. Self-efficacy expectations regarding inconvenience, time and the ability of preparation are important factors that influence the choice whether or not to consume fruit, vegetables and a diet with low-fat products (Brug et al., 1997; Van Assema et al., 2001; Van Duyn et al., 2001; Ma et al., 2002). Perceived self-efficacy concerning the ability to accurately estimate one's portion size influences the energy balance-related behaviour of decreasing portion sizes (French et al., 2001). The most often reported self-efficacy factors influencing walking or cycling to work and being more physically active at work are type of work, lack of time (Urlings et al., 1997; Oja et al., 1998; Proper et al., 1999; Proper et al., 2000; French et al., 2001), and transporting heavy loads (Proper et al., 2000).

Finally, habit has shown to be an important personal determinant of all the specified energy balance-related behaviours. Dietary behaviour, such as eating lots of fruits and vegetables, has often become a habit since childhood (Krebs-Smith et al., 1995; Oja et al., 1998; Baranowski et al., 1999). Eating the same amount of food can also be regarded as a habit, as individuals often eat the same amount of food, without adjusting for the energy content of the food (French et al., 2001; Rolls et al., 2002). Travel behaviour, such as using either the car or bicycle, and using the elevator or stairs are important habitual behaviours that influence the physical activity related health-promoting behaviours (Aarts \& Dijksterhuis, 2000a; Kerr et al., 2001a; Foster \& Hillsdon, 2004).

There is a wide variety of environmental factors that encourage a positive energy balance, sometimes labelled as the 'obesogenic environment' (Swinburn et al., 1999; Davey, 2004). It has been postulated that these factors could have even more influence on people's energy balance-related behaviours than the above mentioned cognitive determinants (Kremers et al., 2005a). A useful framework that can be used to conceptualize obesogenic environments is the ANGELO (Analysis Grid for Environments Linked to Obesity) framework. It is a grid that comprises two sizes of environment (micro and macro) on one axis and four types of environment on the other (Swinburn et al., 1999). In this paper only microenvironments will be discussed, as the intervention is implemented in the worksite setting (i.e. a microenvironment). In ANGELO, types of environment are categorized as physical (what is available), economic (what are the costs), political (what are the rules), or socio-cultural (what are the attitudes and beliefs) (Swinburn et al., 1999). The physical microenvironment includes the availability of bike sheds, changing facilities, cycle paths, sidewalks and continuous routes. These factors have all been identified as determinants of active transport (Oja et al., 1998; Proper et al., 2000; French et al., 2001; Craig et al., 2002; Pikora et al., 2003). Traffic safety (Pikora et al., 2003; De Bruijn et al., 2005), weather conditions, and distance were also identified as physical determinants 
of commuting activities (Proper et al., 2000). Physical micro-environmental determinants that have been shown to influence being physically active at work are the accessibility and attractiveness of stairwells (Sallis et al., 1998; Boutelle et al., 2001) and the proximity to printer, fax and coffee machine (Urlings et al., 1997; Proper et al., 1999; Kahn et al., 2002). The availability of a wide variety of good tasting, inexpensive, energy dense foods (Hill et al., 2003), point-of-purchase information (Seymour et al., 2004) and large portions (Rolls et al., 2002; Rolls et al., 2004) both in vending machines as cafeterias were shown to be important physical determinants of fibre-and fat intake and the consumption of large portion sizes (Glasgow et al., 1997; Sorensen et al., 1998; Beresford et al., 2001).

Important socio-cultural micro-environmental determinants of the discussed energy balance-related behaviours are management commitment and support at all levels of the organization (Hammond et al., 2000; Sorensen et al., 2004). Management commitment may be reflected in the company's mission statement, the budget provided to support initiatives, assignment of staff responsibility for programme oversight and operation, and the involvement of workers in employee advisory boards (Goodman et al., 1997). Support and commitment from management representatives can also reinforce social norms supportive of the energy balance-related behaviours, such as providing healthy food choices in cafeterias and vending machines (Sorensen et al., 2004), and providing bike-sheds and changing facilities. Social support from co-workers and having a companion for physical activity influences walking (Kwak et al., 2006b), active commuting to work and being physically active at work (Urlings et al., 1997; Proper et al., 1999; Ball et al., 2001; Giles-Corti \& Donovan, 2003).

Political micro-environmental determinants refer to rules related to the energy balance-related behaviours and include laws, regulations and policies (Swinburn et al., 1999). Company policies can promote healthy choices or restrict the number of less healthy choices (Engbers et al., 2005), such as obtaining budgets for environmental changes, instituting release time for physical activity, restructuring breaks (e.g. amount of time allowed, timing of the breaks in the day, rules about accessing food during breaks) (Sorensen et al., 2004), including specific statements about providing access to healthy food choices (Simpson et al., 2000) and changing catering policies (Swinburn et al., 1999).

An economic micro-environmental determinant that influences food consumption is food price (French et al., 2001). Cost has been identified as the second most important factor in food decisions, after taste. The influence is however not necessarily health promoting (Glanz et al., 2005), as large price cuts for healthy alternatives may lead to an income effect that can mitigate substitution of unhealthy alternatives (Weber et al., 2005). Another economic micro-environmental determinant is offering incentives for physical activity, such as subsidizing active transport (Sallis et al., 1998).

\section{Step 2: Selecting theory-based intervention methods and practical strategies}

The first task in step 2 of IM is to search for possible theoretical methods of behavioural change. The next task is to translate these methods into practical strategies (Bartholomew et al., 2001).

\section{Theoretical methods}

In order to select suitable theoretical methods to change the determinants specified in step 1 a thorough search of the scientific worksite weight gain prevention literature was conducted. In addition, literature was incorporated on interventions targeting changes in food intake and physical activity behaviours 
and on behaviour change theories. It can be concluded from this search that the underlying theoretical models and methods of behavioural change are rarely fully reported(seealsoKremersetal.,2005b;Kremersetal.,2005c).In a reviewbyHardeman and colleagues (2000), however, a taxonomy of behaviour change methods is proposed that assists individuals in developing interventions directed at weight gain prevention (Hardeman et al., 2000). Behaviour change methods that were selected from this taxonomy and from general behaviour change literature are the following: (self) monitoring, (self) evaluation, personalization of risk, delivering information re-garding behaviour, outcome, and connection between behaviour and outcome, tailoring, decisional balance, skills training (problem solving, decision making, goal setting), prompting, rewarding, environmental changes, social encouragement and social support (Hardeman et al., 2000; Bartholomew et al., 2001). Table 2 gives an overview of the selected behavioural change methods and their underlying theoretical models.

\section{Translating methods into practical strategies}

The second task in IM step 2 is to translate the selected theoretical behaviour change methods into practical personal and work environmental change strategies that will be used in the NHF-NRG In Balance-project (for a detailed overview see table 4a and $4 \mathrm{~b}$ ). In order to make individuals more aware of their own body composition in relation to normative values personalized feedback was given on these measures (Resnicow et al., 2003). Additionally, self-monitoring and self-evaluation practical strategies were provided, not only directed at body composition, but also at physical activity and food intake (Tinker et al., 2001; Rooney et al., 2003).

A practical strategy that was used to deliver information was the launch of a website, as numerous studies have indicated that the Internet has a positive impact on knowledge acquisition (Lewis, 1998). Computer-based technologies also offer possibilities for tailoring. Computer tailoring can provide information, which addresses for example intention, beliefs, social influence, self-efficacy expectations and/or stage of change with respect to different behaviours (Brug et al., 1999; Oenema et al., 2001; Oenema \& Brug, 2003). A recent systematic review of randomized trials on the effectiveness of computer-tailored education on physical activity and dietary behaviours showed significant effects of the tailored interventions, with most consistent evidence for tailored interventions on fat reduction (Kroeze et al., 2006). Tailored information can be provided on multiple behaviours (Calfas etal., 2002; James etal., 2002), for the NHF-NRGIn Balance-project this will be provided on all the energy balance-related behaviours specified in the performance objectives.

Placing prompts at choice points by stairs and elevators was chosen as a strategy to increase stair use, as several studies have shown that point-of-decision prompts increase stair use in several settings, including worksites (French et al., 2001; Titze et al., 2001; Kerr et al., 2004; Webb \& Eves, 2005; Eves \& Webb, 2006a). Point-of-decision prompts were also used as point-of-purchase information in worksite cafeterias and in the form of labelling of healthy food choices (Seymour et al., 2004; Matson-Koffman et al., 2005). Several environmental change strategies are described in the literature related to active commuting and being physically active at work (Oja et al., 1998; Mason, 2000), but also related to changes in food intake (Glasgow et al., 1995; Simpson et al., 2000). Examples of environmental strategies applied in the NHF-NRG In Balance-project are increasing the availability of bike sheds and of low-energy dense products in vending machines.

Practical strategies that were applied to mobilize social support and encouragement from co-workers were the formation of groups of colleagues, such 
as lunch walking groups to stimulate being physically active at work (MatsonKoffman et al., 2005). The formation of an employee advisory board that guides project activities in the worksite and includes representatives from all employee groups and the manager of the cafeteria (Beresford et al., 2001) was used to both increase social encouragement from management and employee involvement (Biener et al., 1999; Simpson et al., 2000; Beresford et al., 2001) (see table 4b).

\section{Step 3: Producing and integrating the intervention programme}

The first task in Intervention Mapping step 3 is to translate the methods and practical strategies selected in Step 2 of IM into programme plans. The product of this step is a plan that outlines scope and sequence of the programme and of all programme vehicles and materials that must be produced (Bartholomew et al., 2001). For the NHF-NRG In Balance-project the methods and practical strategies are depicted in table $4 \mathrm{a}$ and $4 \mathrm{~b}$. Firstly, a brainstorm session with experts was conducted to identify existing intervention materials and activities that fitted the methods and objectives. If necessary, existing materials were adjusted. Finally an overview was made of new materials that needed to be developed.

The programme was a three stage intervention that was arranged into two components, namely an individual component and a worksite (environmental) component (Figure 2). The individual component contained the following materials: feedback on measured BMI, fat percentage and waist circumference sent by e-mail; an 'In Balance-box' consisting of a pedometer, waist circumference measuring tape, a "calorie guide", and an instruction brochure, which included a physical activity diary, a food intake diary, log of steps walked and instructions on how to use these devices; an In Balance-website with access to the Weight-Co@ch; computer-tailored CD-ROM I, which was directed at awareness of weight status and knowledge regarding the energy balance-related behaviours; computer-tailored CD-ROM II, which was directed at changing the energy balance-related behaviours (table 4a). According to the literature a promising approach is tailoring on the participant's choice of behavioural priority for change (Campbell et al., 2000), we therefore decided to incorporate this into our computer-tailoring programme in such a way that individuals could choose the energy balance-related behaviour that they would like to change first.

The environmental components (table $4 \mathrm{~b}$ ) were incorporated into a handbook, namely the 'weight gain prevention at the worksite' handbook, which contains the existing 7-step programme of the Workplace Health Promotion consultancy (The Netherlands Institute for Health Promotion and Disease Prevention (Healthcare and Work)) and interventions directed at the energy expenditure-related behaviours, which included posters/prompts stimulating stair use, ways to form lunch walking and cycling groups and the "bike to work" campaign (COS Netherlands). 


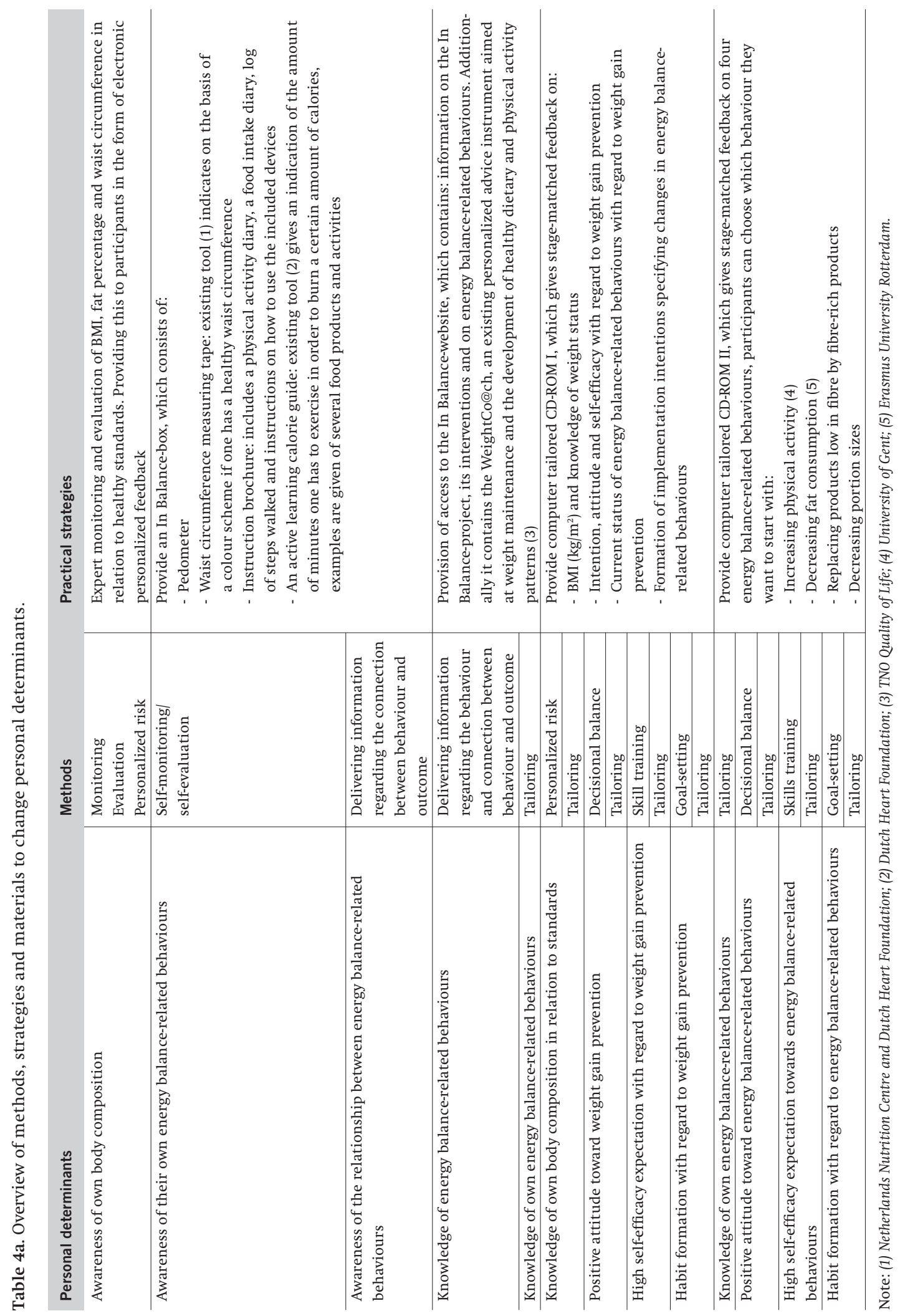




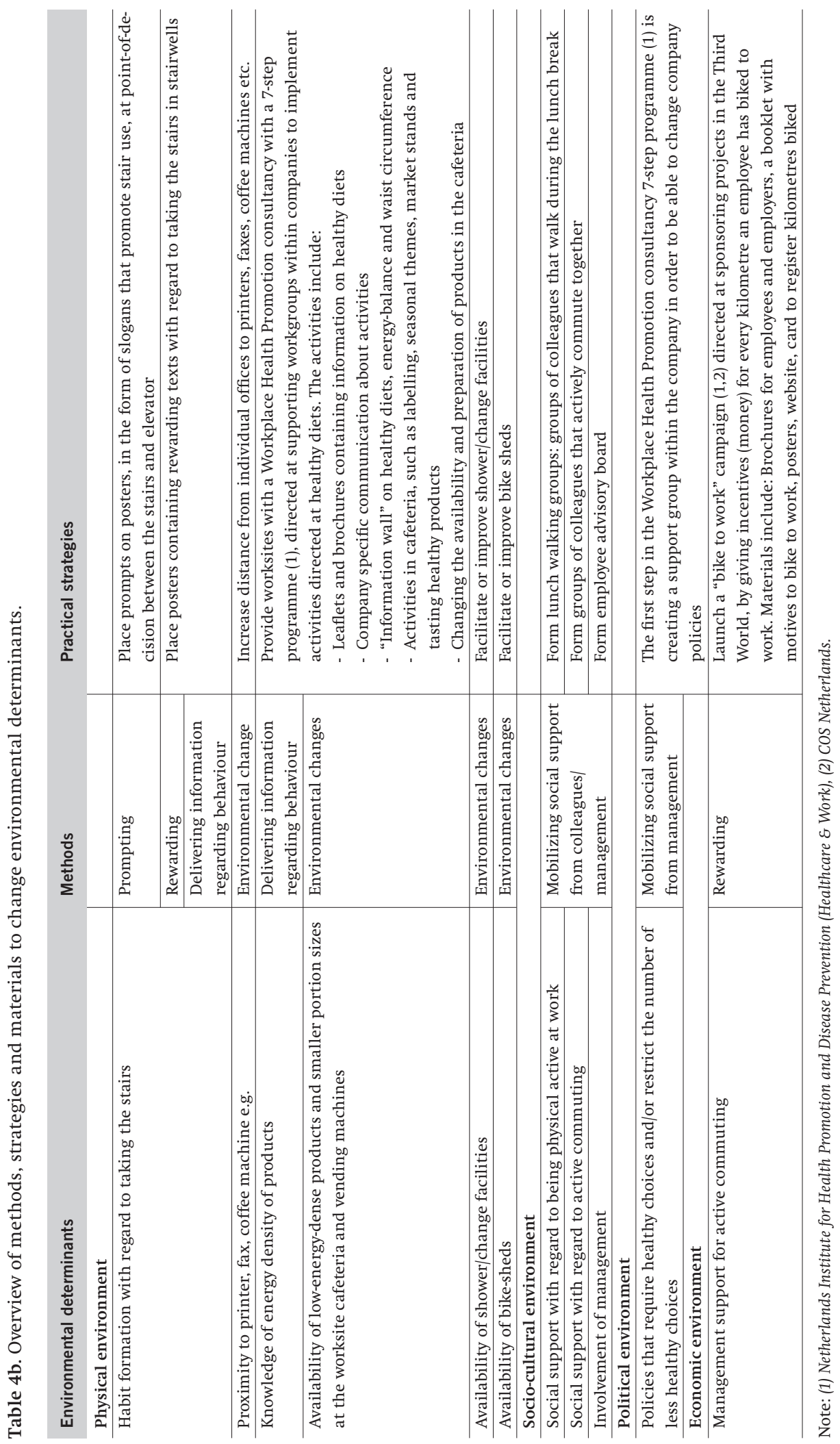


Figure 2. Three stage intervention sequence.

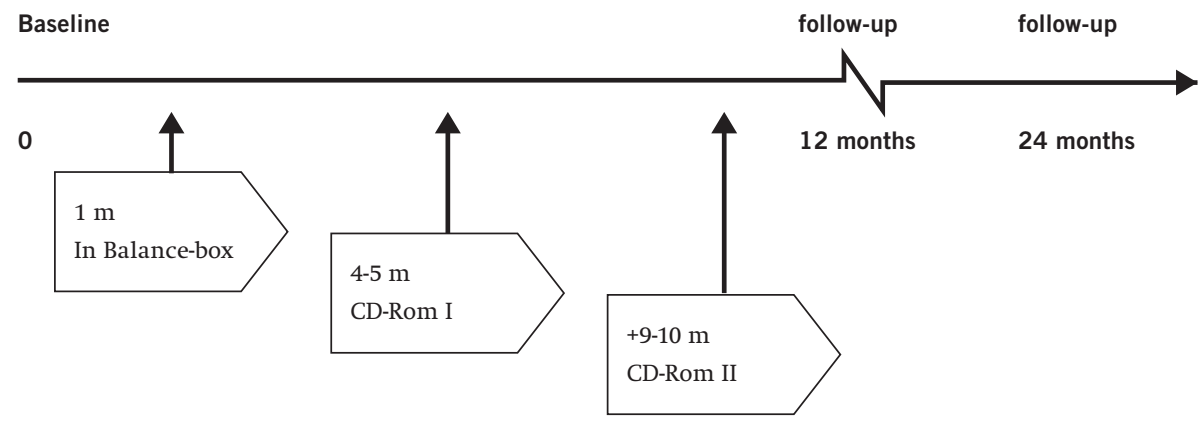

In Balance website (Weighco@ch)

Environmental interventions

\section{Step 4: Adoption and implementation plan}

The focus of step 4 is on the planning of an intervention in order to ensure that the programme developed in the previous steps will be implemented, adopted and sustained over time. The formation of a linkage system, which connects the developers of the intervention to the users, has been argued to stimulate this process of diffusion, adoption and implementation (Bartholomew et al., 2001).

Within each worksite, a linkage board was formed that comprised of a representative of the research team and employees who were able to facilitate collaboration or were in positions to influence changes necessary to support the adoption, implementation, and institutionalization of the programme, such as for example representatives of the worksite cafeteria and/or catering. The linkage board was responsible for the adoption and implementation of the worksite-based interventions. The handbook: 'weight gain prevention at the worksite' served as a guide for assisting the linkage board through the different stages of diffusion. In order to optimally diffuse the programme, interventions were selected that match the needs and possibilities of the worksite. Accordingly, interventions were modified to fit within each specific worksite. In order to increase sustainability of the interventions, also after the research period, the representative of the research team was only present the first year of the programme and reduced input as the year evolved.

Additionally, materials were pre-tested in order to further improve the chances for adoption and implementation.

\section{Step 5: Monitoring and evaluation plan}

The development of an evaluation plan is the final step in IM (Bartholomew et al., 2001). The evaluation plan of the present study includes a process and effect evaluation (figure 3). The Environmental Research framework for weight Gain prevention (EnRG) formed the basis for the evaluation model (see Kremers et al., 2006 for more information). The process evaluation was primarily based on Rogers' (1995) diffusion of innovations-model and included monitoring of the intervention delivery, participation, comprehension, satisfaction, level of use, fidelity and institutionalization (Rogers et al., 1995). Indicators of success were divided into indirect, intermediate and outcome indicators (Tones, 2000). Techniques that were used to 
collect this information included observations, interviews with key-actors and selfadministered questionnaires for the participating young adults. To evaluate the effect of the intervention outcome measures were used that are related to the objectives stated in the needs assessment and IM Step 1. In order to determine the efficacy of the intervention a quasi-experimental pre-test-multiple post-test control group design was applied. In total 12 worksites (>500 young adult employees) were recruited, which was a sufficiently large enough sample size to be able to detect a difference of $0.6 \mathrm{~kg}$ in average body weight over 1-year between the experimental and control group. The difference of $0.6 \mathrm{~kg}$ is based on the expected weight gain in this age group in the Netherlands in one year (Visscher et al., 2002). Accordingly, worksites were matched with respect to social economic status of the employees and assigned to either the experimental or control group (see also Kwak et al., 2006a).

The main outcome measure was change in body weight, but skinfold thicknesses, and waist and hip circumference were also included as physiological outcomes, as the latter three can change regardless of changes in body weight, but as a result of changes in fat mass (Brodie et al., 1998; Sarria et al., 1998; Seidell et al., 2001). Cognitions, intentions and energy balance-related behaviours were measured with questionnaires. More valid but expensive and time-consuming instruments were selected to measure physical activity behaviour (24h recall, MTI-accelerometer), body composition (underwater weighing, deuterium dilution) and fitness (VO2-max test) in a sub sample of the participants (approximately $10 \%$ of the total sample).

Outcome measures were collected in the experimental and control group at baseline (before the start of the intervention), at the end of the 1-year intervention, and one year after the end of the intervention. In the experimental group this also included the collection of process measures. For the participants, process evaluation items were included in the questionnaires that also assessed cognitive factors and behaviours. Although our main interest was to establish the overall effectiveness of the intervention, we also included process measures in our evaluation design, in order to gain insight into the effectiveness of specific ingredients of the overall intervention. Additionally, experimental and control worksite environments were observed in order to assess characteristics of worksite environments that were known to influence health-related behaviours, this was done with an adapted version of the Checklist of Health Promotion Environments at Worksites (CHEW; Oldenburg et al., 2002).

\section{DISCUSSION}

The purpose of the present paper was to present the development and implementation of the NHF-NRG In Balance-project, providing information on the underlying rationale, theory, behaviour change methodology, interventions and evaluation design. To date, relatively few weight gain prevention studies have provided details on these matters (Hardeman et al., 2000). With this paper we therefore aimed at contributing to the shortage in this field.

In the present study IM was not completely applied according to the protocol, as matrices of learning and change objectives, as prescribed by the IM protocol, were not created. The choice to apply a modified version of IM is a result of the complexity of weight gain prevention. IM is often applied to the development of interventions that are directed at changing relatively simple, more uni-dimensional behaviours, such as the promotion of fruit and vegetable consumption in schoolchildren (Perez-Rodrigo et al., 2005) and stimulating condom use among drug users 
Figure 3. NHF-NRG In Balance-project intervention and evaluation model (adapted version of the-figure by Kremers et al., 2005a).

INDICATORS OF SUCCES

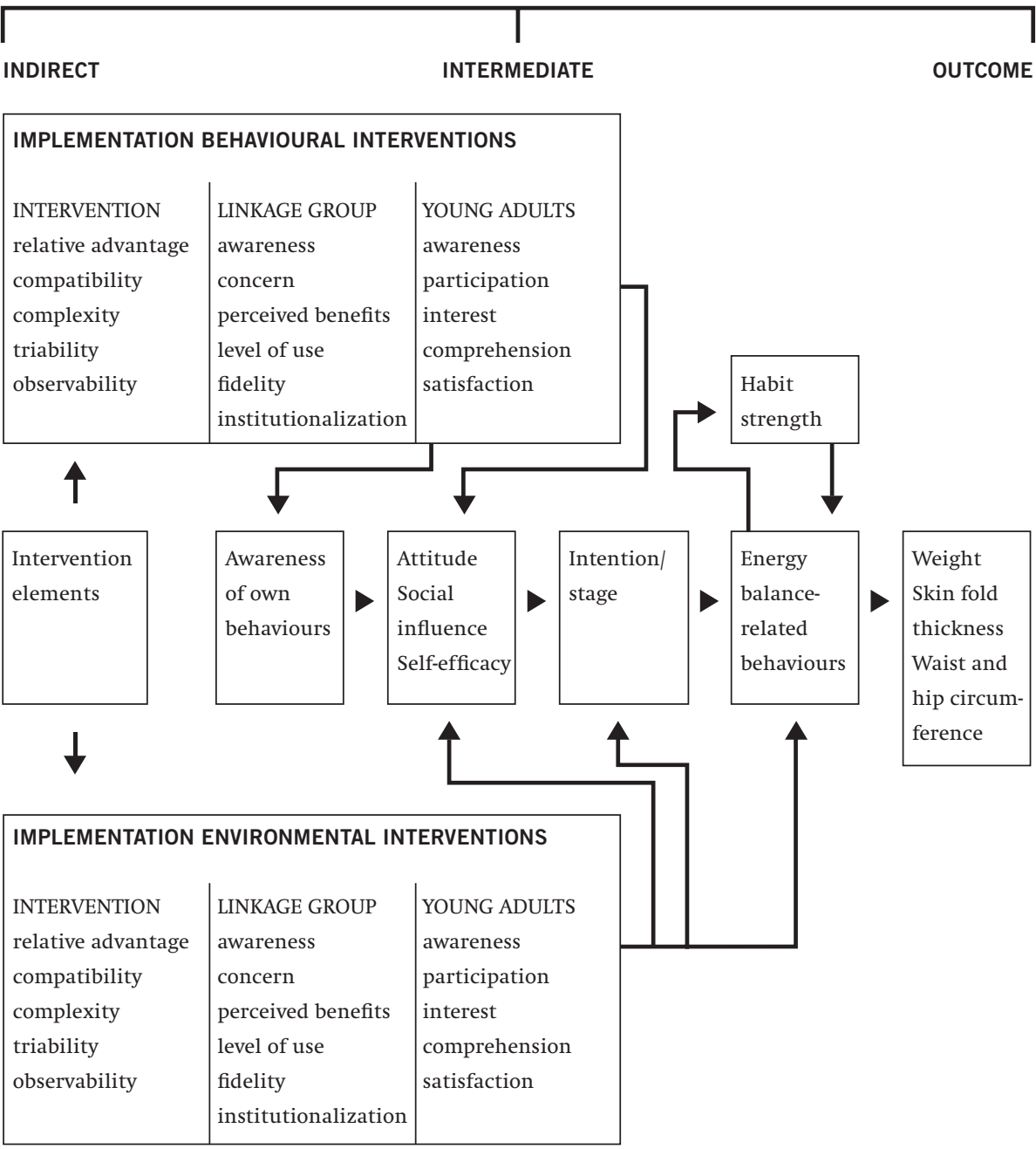

(Van Empelen et al., 2003). As the prevention of weight gain involves several energy balance-related behaviours, each of which has their own distinct cognitive and environmental determinants (Kremers et al., 2005a), it would have been impractical to create learning and change objectives for all the identified determinants. In order to restrict the amount and complexity of matrices, it was decided to guide the specification of the performance objectives according to the self-regulation theory and implementation intention theory. This modification has also been made in other interventions that applied the IM protocol to complex behaviours with satisfactory results (Martens et al., 2006).

Although the original design of the programme included randomization, companies were not randomly assigned to the experimental and control group, but matched. An important reason for companies not to participate in the NHF- 
NRG In Balance-project proved to be the randomized evaluation design of the programme, implying that companies were not willing to take the risk of being excluded from the intervention. As a result, we were forced to drop the original randomization design of the programme (Kwak et al., 2006a).

Apart from the theoretical and empirical based design of the NHF-NRG In Balance-project there are several other potential strengths of the programme. Firstly, this is one of the few programmes aimed at the prevention of weight gain (Hardeman et al., 2000). Previous interventions aimed at the prevention of overweight and obesity in worksite settings have mainly focused on weight loss and not on primary obesity prevention (Katz et al., 2005). Another potential strength of the programme is that it is a multi-component intervention focusing on both nutrition and physical activity. Katz and colleagues (2005) concluded after their systematic review of the evidence on effectiveness of interventions for preventing and controlling overweight and obesity in worksite settings, that there is insufficient evidence to determine the effectiveness of single-component worksite interventions and recommend that emphasis should be placed on interventions combining nutrition and physical activity (Katz et al., 2005). The present study does not just combine the two, but also gives participants the opportunity to choose the energy balance-related behaviours that they would like to change. It has been suggested that giving participants this opportunity will more likely lead to behaviour change (Kremers et al., 2005d). Additionally, it has been shown that clustering occurs both within energy balance-related behaviours as within their cognitive determinants, suggesting a synergistic cognitive motivational mechanism within these behaviours (Kremers et al., 2004). The NHF-NRG In Balanceproject is directed at both the individual and the environment, thus stimulating and facilitating behaviour change at the same time.

In conclusion it can be stated that the explosive increase in the prevalence of obesity, its associated serious medical problems and its complexity demands more than just weight loss programmes and weight gain prevention programmes with no theoretical or empirical foundation. A systematic approach to intervention development, implementation and evaluation is needed in order to optimally document effective methods, successful strategies and pitfalls. Although this may require more time and effort, a thorough documentation and publication of this process, will eventually lead to more cost-efficiency with regard to tackling obesity. Major challenges in our project were the recruitment of companies willing to participate in a randomized study design and incorporating the complexity of energy balance-related behaviours in the systematic approach of IM. We feel that we have identified satisfactory solutions, i.e., the application of a matched design and the use of self-regulation theory to reduce amount and complexity of matrices that may be helpful in the design of future intervention studies.

\section{GENERAL OUTLINE OF THE THESIS}

In this thesis studies are presented that are related to the development, implementation and evaluation of the NHF-NRG In Balance-project, with the overall aim of testing the effectiveness of the programme. Chapter 2 elaborates on the recruitment problems that occurred before the programme was implemented. Next the results are presented from a study exploring the accuracy of the physical activity questionnaire that was used to evaluate the behavioural physical activity outcomes of the intervention. Accordingly three studies are presented that test the effectiveness of one of the goals of the programme, namely promoting stair use at work. Chapter 
4 examines whether an intervention using implementation intentions is effective in stimulating stair use in a worksite setting. The long-term effectiveness of the implementation intentions are examined in chapter 5. Chapter 6 presents the results of a study testing the effectiveness of an intervention using prompts on posters in stimulating stair use in two types of worksites; one consisting mainly of white-collar workers and one mainly of blue-collar workers. Chapters 7 and 8 examine the short-term and longer term effectiveness of the programme. The results regarding the changes in anthropometric measures are presented in chapter 7 , while the changes in weight gain preventive behaviours, motivational stages and corresponding cognitive factors are presented in chapter 8.

The results of all the presented studies are discussed and integrated in the General Discussion. Furthermore methodological and intervention-related issues are discussed in this chapter, as well as implications for practice and future research. 


\section{CHAPTER 2}

\section{PARTICIPATION RATES IN WORKSITE-BASED INTERVENTION STUDIES: HEALTH PROMOTION CONTEXT AS A CRUCIAL QUALITY CRITERION}




\section{ABSTRACT}

Recently, a set of specific quality evaluation criteria for health promotion research has been proposed in this journal. One of the quality criteria identified is the 'health promotion context'. With this paper we would like to contribute to the dialogue by specifying the importance of this criterion on the basis of our own experience with worksite-based obesity prevention interventions. We advocate the reporting of participation rates among approached worksites in publications on worksite intervention effects. Such information will help to draw conclusions on the practical relevance of the shown effectiveness of the intervention. Health promotion practice is advised to adopt and disseminate evidence-based interventions, accompanied by a diffusion study with a minimal research burden for participants. 
Lahtinen et al. (2005) have convincingly argued that widely acknowledged and accepted quality standards for health promotion research are urgently needed in order to improve quality and effectiveness of interventions. They have therefore developed a set of specific quality evaluation criteria for health promotion research, which are intended to guide funding agencies, researchers, evaluators and reviewers (Lahtinen et al., 2005). As a consequence of recent experiences in the field of worksite-based health promotion, we were especially impressed by Lahtinen et al.'s identification of 'Health Promotion Context' as a quality criterion. The authors have postulated the need for a dialogue on health promotion quality criteria and we fully acknowledge the triviality of such discussions. With this paper we would like to specify the importance of the health promotion context as a quality criterion and for this purpose, we will take our recent experiences with worksite-based obesity prevention interventions as an example. Specific recommendations are put forward for journal editors, researchers and health promotion practice.

In recent years worksites have often been viewed as ideal settings for health promotion programmes (Sorensen et al., 1999). Not only do worksites provide an easy longitudinal access to a large number of people, they also offer the possibility to conduct multi-level interventions, directed at individual, organisational and environmental determinants of health and health behaviours.

Matson-Koffman and colleagues have recently reviewed the literature to determine whether policy and environmental worksite interventions can increase people's physical activity or improve their dietary habits. They provided a thorough description of studies published during 1990-2003, including information on the authors, the setting and location, the size and characteristics of the sample, the intervention itself, the evaluation period and the actual findings. In total, details were given on 18 worksite-based interventions directed at either physical activity or nutrition. Matson-Koffman and colleagues concluded that worksite-based interventions are effective in changing physical activity and nutrition behaviours of the working population (Matson-Koffman et al., 2005). But are the effects of worksite interventions as promising as their and other reviews (Glanz et al., 1996; Hennrikus \& Jeffery, 1996; Shephard, 1996; Pelletier, 1999; Emmons et al., 2000; Aldana \& Pronk, 2001; Seymour et al., 2004; Sorensen et al., 2004; Matson-Koffman et al., 2005) suggest?

According to Bull and colleagues reviews of evaluations of worksite health promotion interventions should not just focus on internal validity and effect sizes of the results. In fact, high external validity is needed to facilitate implementation of proven and broadly applicable interventions (Bull et al., 2003). External validity depends largely on the extent to which the studied population is a representation of the total population. In order to make a judgement of the external validity of intervention effects, the recruitment procedure of the studied worksites needs to be accurately described. Bull et al. (2003) showed that only six out of the 24 worksitebased intervention studies in their review offered information on the proportion of participating sites from those eligible to participate (Bull et al., 2003). Furthermore, only one of these six studies reported the number of worksites that were originally approached to participate in the study. The adoption participation rate in this particular study was $39.0 \%$. We have examined the level of reporting of the adoption participation rate in the worksite-based studies included in Matson-Koffman et al.'s review. Remarkably, only one out of the 18 studies reported the total number of companies originally approached to participate, with an adoption participation rate of $61.9 \%$.

The general lack of reporting of participation rates may reflect low levels of participation among approached worksites. In medical research, the CONSORT 
statement (Moher et al., 2001) is increasingly accepted in reporting Randomised Controlled Trials. The CONSORT, which includes a flow chart providing readers with a clear picture of the progress of the participants in the trial, has been endorsed by prominent medical journals such as The Lancet, Annals of Internal Medicine, and the Journal of the American Medical Association. Health promotion journals should consider implementing similar guidelines regarding in-depth reporting of the research process, giving readers the possibility to appropriately evaluate its validity.

Our own experience with worksite health promotion studies in the Netherlands, especially in the recent years of low economic growth that we experienced in the European Union (Gateway to the European Union, 2005), indicates that low participation rates among worksites approached for participation in health promotion programmes are common. For our worksite-based programme aiming to prevent unnecessary weight gain in workers, the NHF NRG-In Balance-project, which is part of the larger Netherlands Heart Foundation's-NRG study (Kremers et al., 2005a), we approached 128 companies to participate. Only 12 of those companies (9\%) were willing to participate. Three other large Dutch worksite health promotion programmes experienced similar recruitment problems and reported participation rates among worksites of $18 \%^{\mathrm{a}}, 16 \%^{\mathrm{b}}$ and $22 \%^{\mathrm{c}}$, respectively.

The most important reasons for refusal to participate, in our experience, were lack of time and resources in times of economic stagnation and unwillingness to be subjected to the demands of research. One of these demands is randomisation, which implies that companies need to take the risk of being excluded from the intervention. As a result, companies known to be collaborative may be sequentially approached for participation in different studies, or relatively cooperative federal organisations are over-sampled (Larsen \& Simons, 1993; Titze et al., 2001; Yancey et al., 2004). Such procedures may introduce serious threats to external validity.

The consequences of low external validity are as obvious as expensive: nationally implemented interventions that have shown to be effective in a trial may encounter many barriers in the dissemination process. The intervention could be effective in the few companies that are interested, but most worksites may simply not adopt the intervention. However, real-world diffusion studies are needed to learn about the exportability and adoption of interventions in less controlled conditions than those accompanied with trials (Harting et al., 2005). Oldenburg and colleagues have argued that less than $1 \%$ of all public health and health promotion studies are categorized as diffusion research (Oldenburg et al., 1999). Controlled trials with good process measures provide information on the potential effectiveness of intervention elements. Dissemination studies are urgently needed to inform us on the adoption and efficacy of successful intervention elements in health promotion practice. Only the supplementation of dissemination studies to controlled trials will illuminate the usefulness of interventions in daily health promotion practice. The research burden for participants should be minimized in order to coincide with the 'real world'.

Consequently, we advocate the reporting of participation rates among approached worksites in publications on worksite intervention effects as well as indicating expected participation rates in research proposals. Such information will help to draw conclusions on the practical relevance of the shown effectiveness of the intervention, and thus, the evidence-base in health promotion will be optimised. Health promotion practice is advised to adopt and disseminate evidence-based interventions, accompanied by a diffusion study with a minimal research burden for participants.

Lahtinen et al.'s identification of 'Health Promotion Context' as a quality criterion entails that research should demonstrate appreciation for the manner and 
degree to which it is embedded in a larger health promotion context, by reference to critical aspects of the problem that are not objects of study (Lahtinen et al., 2005). We feel that reporting of participation rates among approached worksites in publications as well as indicating expected participation rates in research proposals would optimize the relevance of this quality criterion.

\section{FOOTNOTES}

a Computer tailored information to influence nutrition, smoking and exercise habits (advice about lifestyle through tailoring). Smeets, T. Department of Health Education and Promotion, Maastricht University, the Netherlands

b Testing the efficacy of computer-tailored nutrition education at the worksite. Kroeze, W. Department of Public Health, Erasmus Medical Centre, Rotterdam, the Netherlands

c Promotion of a physically active lifestyle among inactive adolescents and young adults by means of an activity monitor and an individually tailored advice using internet technology. Slootmaker, S. Department of Social Medicine, EMGO Institute, Free University Medical Centre, Amsterdam, the Netherlands 


\section{CHAPTER 3}

\section{MEASURING PHYSICAL ACTIVITY IN FIELD STUDIES: COMPARISON OF A QUESTIONNAIRE, 24H RECALL AND ACCELEROMETER}




\section{ABSTRACT}

The present study compared a physical activity (PA) questionnaire (SQUASH), a $24 \mathrm{~h}$ recall measure (Physical Activity Scale; PAS) and accelerometer for (1) quantifying the amount of time spent in light, moderate and vigorous physical activity, (2) calculating activity scores and (3) categorizing subjects in physical activity tertiles, in a field setting during 7 days. For 7 consecutive days, 103 participants (61\% men; mean age 36.8 years; mean BMI $26.6 \mathrm{~kg} / \mathrm{m}^{2}$ ) wore an accelerometer and completed a PAS. Immediately before these 7 days, participants completed the SQUASH and underwent anthropometrical measurements (weight and height). Measured time spent in light (<3METs), moderate (3-6METs) and vigorous ( $\geq 6 \mathrm{METs})$ activity was calculated. Significant correlations were observed between PAS and accelerometer in the overall population $(\mathrm{r}=.66, \mathrm{p}<.001)$, men $(\mathrm{r}=.76, \mathrm{p}<.001)$, normal weight $(\mathrm{r}=.62, \mathrm{p}<.05)$ and overweight participants $(\mathrm{r}=.53, \mathrm{p}<.01)$. Correlations between SQUASH and accelerometer were only significant for men $(\mathrm{r}=.62, \mathrm{p}<.05)$ and overweight individuals $(\mathrm{r}=.40$, $\mathrm{p}<.05$ ). Moderate agreement was observed between tertiles of accelerometer and PAS activity scores in the overall population (weighted Kappa .47; 95\% C.I.=.27-.67) but not between accelerometer and SQUASH. In conclusion, a 7-day administration of the PAS is a useful measure for classification of physical activity levels, in the studied population. The SQUASH has practical advantages in terms of convenience of administrating, but its usefulness in estimating PA levels is limited. 


\section{INTRODUCTION}

Regular physical activity has important health benefits, including a decrease in the risk of obesity, coronary heart disease, type-2 diabetes mellitus, osteoporosis and certain types of cancer (Norman et al., 2001). Despite a thorough documentation of the benefits of physical activity, accurate assessment of physical activity seems more critical, as an optimum tool for assessing physical activity does not exist (Livingstone et al., 2003). Depending on the instrument used estimates of the prevalence of limited physical activity in women, for example, have varied from 3.9\% to 39.0\% (Shephard, 2003). The 'gold standard' for the measurement of energy expenditure is the Doubly Labelled Water (DLW) method (Schoeller \& Delany, 1998; Speakman, 2001). However, DLW only allows for measurement of total energy expenditure and due to its high cost and technical complexity it is less applicable in large-scale studies and mainly used as reference technique for validating other measures of physical activity (Ainslie et al., 2003). Another objective measure, which provides a more detailed record of physical activity, is the accelerometer (Welk et al., 2000). These devices enable detection and recording of the magnitude of accelerations, most commonly in the vertical plane. They can store data continuously over long periods of time and provide objective assessments of the frequency, intensity, and duration of physical activity performed (Hendelman et al., 2000). Although such activity monitors offer considerable promise for providing valid assessments of physical activity, their application in large-scale studies is constrained by cost and technical complexity (Livingstone et al., 2003). Epidemiological studies often use self-report measures to assess physical activity levels and to categorize individuals in high and low levels of physical activity (Wendel-Vos et al., 2003). As with other complex behaviours, the development of an accurate selfreport measure is difficult to achieve (Chasan-Taber et al., 2002), especially one that is applicable to a diverse population. Current physical activity measures may therefore misclassify physical activity levels for certain subpopulations, such as women, as the majority of physical activity measures has been developed and validated in men with an emphasis on participation in moderate to vigorous sports (Ainsworth et al., 2000; Chasan-Taber et al., 2002). The validity of physical activity measures may also differ by relative weight of the participants (Norman et al., 2001). The few studies that have reported the validity and reliability of self-report measures of physical activity among overweight and non-overweight individuals observed differences according to weight status (Norman et al., 2001; Timperio et al., 2003).

Two distinct types of self-report instruments for measuring physical activity are the Short QUestionnaire to ASsess Health-enhancing physical activity (SQUASH) (Wendel-Vos et al., 2003) and the Physical Activity Scale (PAS) (Aadahl \& Jorgensen, 2003). The SQUASH is developed by the Dutch National Institute of Public Health and gives an indication of habitual physical activity and assesses compliance to physical activity guidelines (Wendel-Vos et al., 2003). The PAS is designed as a $24 \mathrm{~h}$ recall and assesses total physical activity in the past 24 hours (Aadahl \& Jorgensen, 2003). Up to now, both methods have only been validated against an accelerometer in an overall population and the PAS only over a 1-day period (Aadahl \& Jorgensen, 2003; WendelVos et al., 2003). The primary purpose of the present study was to compare a single application of the SQUASH and a 7-day application of the PAS against objective data derived from an accelerometer in a field setting during a 7-day period in an overall sample, in males and females separately and in normal weight and overweight individuals. More specifically, a comparison was made between the three methods for (1) quantifying the amount of time spent in light, moderate and vigorous physical activity, (2) calculating the activity scores and (3) categorizing subjects in physical activity tertiles. 


\section{METHODS}

\section{Participants and study design}

A total of 103 healthy study participants (63 men and 40 women) were recruited on a voluntary basis from the NHF-NRG In Balance-project, a worksite-based health promotion programme to prevent weight gain (Kremers et al., 2005a). No considerations were made regarding gender, age and BMI distribution. All participants read and signed an approved informed consent form before participating in the study. The Medical Ethical Committee of the Academic Hospital Maastricht, the Netherlands, approved the protocol.

The research team visited the participants on three occasions. At the first visit anthropometric measurements were executed. During the second visit participants received the SQUASH (Wendel-Vos et al., 2003), PAS's (Aadahl \& Jorgensen, 2003) and an accelerometer (Computer Science and Applications Inc. Activity monitor operator's manual, 1999). Participants completed the SQUASH immediately before they started wearing the accelerometer. During the 7-day period that participants wore the accelerometer they were asked to fill in a PAS at the end of each day. At the final visit participants returned their self-report measures and accelerometer.

\section{Anthropometric measurements}

Height (m) was measured to the nearest $1.0 \mathrm{~mm}$ without shoes with a mobile measuring unit (Seca, Model 225, Hamburg, Germany) and weight ( $\mathrm{kg}$ ) was measured, in underwear, to the nearest $0.1 \mathrm{~kg}$ with a digital laboratory scale (Seca, Model 861, Hamburg, Germany).

\section{MTI Actigraph accelerometer}

The MTI Actigraph Activity Monitor (Manufacturing Technology Inc Actigraph (model AM7164)) is a small (5.1 x $3.8 \times 1.5 \mathrm{~cm})$, lightweight $(45 \mathrm{~g})$, single channel uniaxial accelerometer, which is designed to measure and record accelerations ranging in magnitude from approximately 0.05 to 2 times gravitational acceleration (Computer Science and Applications Inc. Activity monitor operator's manual, 1999). The monitor was set to accumulate the number of counts in 1-min epochs, beginning at the time the monitor was programmed to start and finishing after seven days when the researcher manually stopped the monitor. The accelerometer was worn around the waist (sagittal line) against the body on the back, using an elastic strap with an adjustable buckle. Participants were instructed to wear the accelerometer during waking hours for the 7-day study period, excluding periods of bathing or other water activities. The data were stored in the memory of the monitor and downloaded to a computer using a reader interface unit. All data were imported into Microsoft Excel for further processing.

In order to compute the average minutes per day spent in light (<3METs), moderate (3-6METs) and vigorous ( $\geq 6.0 \mathrm{MET})$ activities, activity counts were converted to MET values using the equation of Swartz et al. (MET $<3$ ( $\leq 573$ counts/min); MET 3-6 (574-4944 counts/min); MET $\geq 6$ ( $\geq 4945$ counts/min)) (Swartz et al., 2000). In addition, the mean activity counts expressed as counts per minute were calculated by dividing the total activity counts over 7 days by the total number of minutes that the monitor was worn. For the purpose of this study mean activity counts will be referred to as the accelerometer activity score.

Subjects were excluded from the analysis if the accelerometer was not worn for at least 12 hours each day of 7 measurement days (Wendel-Vos et al., 2003). These periods were identified by long runs of zero's in the Excel sheet. Additionally participants indicated on their daily PAS when they had not worn their accelerometer. 


\section{Short QUestionnaire to ASsess Health-enhancing physical activity (SQUASH)}

The SQUASH contains questions on commuting activities (A), leisure time activities (B), household activities (C) and activities at work (D). The reference period is a typical week during the last few months. In parts (A) and (B) each question consists of three main queries: days per week, average time per day, and effort. Effort refers to the intensity of the activity; participants indicate how strenuous the activity was, this could either be light (slow), moderate or vigorous (fast). In parts $(C)$ and (D) intensity was pre-structured into just two categories, either light or vigorous activities. To make it easier for participants to know which type of activities were applicable, examples were given for the questions that referred to household activities, activities at work and sports. Completing the SQUASH takes about 3-5 minutes (Wendel-Vos et al., 2003).

In order to calculate the average minutes per day spent in light (<3METs), moderate (3-6METs) and vigorous ( $\geq 6.0 \mathrm{MET})$ activities, activities were subdivided into these three intensity categories using Ainsworth's Compendium of Physical activities (Ainsworth, et al., 1993). Total minutes of activity were calculated for each question by multiplying frequency (days/week) by duration (min/day). Activity scores for separate questions were calculated by multiplying total minutes of activity by an intensity score (ranging from 1 to 9). The total activity score was calculated by taking the sum of the activity scores for the separate questions. According to the protocol of the SQUASH, data were excluded from the analysis if the total minutes of activity per day exceeded 960 minutes (Wendel-Vos et al., 2003).

\section{Physical Activity Scale (PAS)}

The PAS lists activities in nine levels of physical exertion, ranging from sleep or inactivity (0.9 METs) to strenuous activities (>6 METs) (Aadahl \& Jorgensen, 2003). Each level was described by examples of specific activities of that particular MET level and by a small drawing. The PAS was constructed in such a way that the number of minutes $(15,30$, or 45 minutes) and hours (1-10h) spent on each MET activity level could be filled out. This allowed for a calculation of the total MET-time representing 24 hours of sleep, work and leisure time. For the purpose of this study the PAS was translated into Dutch and cycling was included as a separate category, as cycling is an activity, which is regularly performed in the Netherlands. Completing the PAS takes approximately 5-10 minutes each day.

The MET-time was calculated from the PAS by multiplying time spent on each activity level by the MET value of each level. MET time from all nine levels was added to a total 24-h MET time (Aadahl \& Jorgensen, 2003). For the purpose of this study total 24-h MET time will be referred to as the PAS activity score.

\section{Statistical analysis}

Data were analyzed using SPSS 11.5 statistical package. Descriptive analyses were run regarding time spent in physical activity and differences between instruments in mean time spent in moderate activity were tested using t-tests. The association between the accelerometer and the SQUASH and PAS was explored by assessing Spearman rank-order correlations between the activity scores calculated through the accelerometer and SQUASH and PAS. Linear regressions were performed between the activity scores of the SQUASH and accelerometer and the activity scores of the PAS and accelerometer. Gender and body mass index (BMI; calculated as weight divided by height squared) differences in the associations between the physical activity scores measured through the self-report measures and accelerometer, were assessed by including the main effects for gender and BMI with both the SQUASH 
and PAS in each linear regression model. In case of significant main effects, the interaction of gender and BMI was added to the regression equation.

The agreement in categorization of subjects in physical activity tertiles between the accelerometer activity scores on the one hand and the SQUASH and PAS activity scores on the other was examined by calculating the linear weighted kappa statistic (Brenner \& Kliebsch, 1996). Kappa interpretations were based on Landis and Koch's (Landis \& Koch, 1977; Field 2003) guidelines $(\leq 0=$ poor, $.01-.20=$ slight, $.21-.40=$ fair, .41-.60=moderate, .61-.80=substantial, and $.81-1=$ almost perfect). Additionally, separate analyses were conducted by gender and by weight status (normal weight $=\mathrm{BMI}<25 \mathrm{~kg} / \mathrm{m}^{2}$; overweight $\left.=\mathrm{BMI} \geq 25 \mathrm{~kg} / \mathrm{m}^{2}\right)$.

\section{RESULTS}

Men were significantly older than women $(\mathrm{p}<.001)$ and had significantly higher mean BMI's than women ( $\mathrm{p}<.001)$ (Table 1). Respectively $96(93.2 \%)$ and $101(98.1 \%)$ participants completed the SQUASH and PAS accurately. Valid accelerometer data were available for 43 participants (41.7\%; 25 men and 18 women), due to exclusion of participants that did not wear the accelerometer for a minimum of 12 hours a day for a period of 7 days. The main reason participants reported for not wearing the accelerometer was inconvenience. There was no significant difference in gen$\operatorname{der}\left(\mathrm{X}^{2}=.284\right.$, ns), BMI $\left(\mathrm{X}^{2}=.208\right.$, ns) or self-reported physical activity levels, measured through either the SQUASH or PAS, between the group of participants that wore the accelerometer correctly and incorrectly.

Table1. Descriptive characteristics and minutes of physical activity per dayin intensity categories, measured by SQUASH $(n=96)$, PAS $(n=101)$ and accelerometer $(n=43)$ in total sample, and in gender and BMI groups.

\begin{tabular}{|c|c|c|c|c|c|}
\hline & $\begin{array}{l}\text { Total } \\
(n=103)\end{array}$ & $\begin{array}{l}\text { Men } \\
(n=63)\end{array}$ & $\begin{array}{l}\text { Women } \\
(n=40)\end{array}$ & $\begin{array}{l}\text { BMI }<25 \mathrm{~kg} / \mathrm{m}^{2} \\
(\mathrm{n}=41)\end{array}$ & $\begin{array}{l}\mathrm{BMI} \geq 25 \mathrm{~kg} / \mathrm{m}^{2} \\
(\mathrm{n}=62)\end{array}$ \\
\hline Age (years) & $36.8 \pm 7.3$ & $38.8 \pm 7.5$ & $33.7 \pm 5.8^{* * *}$ & $34.8 \pm 6.1$ & $38.1 \pm 7.7$ \\
\hline BMI (kg/m²) & $26.6 \pm 4.3$ & $27.7 \pm 4.7$ & $24.8 \pm 2.9^{* * *}$ & $22.9 \pm 1.5$ & $29.0 \pm 3.9$ \\
\hline $\begin{array}{l}\text { SQUASH } \\
\text { (min/day) }\end{array}$ & $\begin{array}{l}\text { Total } \\
(n=96)\end{array}$ & $\begin{array}{l}\text { Men } \\
(n=60)\end{array}$ & $\begin{array}{l}\text { Women } \\
(n=36)\end{array}$ & $\begin{array}{l}\text { BMI<25kg/m2 } \\
(n=36)\end{array}$ & $\begin{array}{l}\mathrm{BMI} \geq 25 \mathrm{~kg} / \mathrm{m} 2 \\
(\mathrm{n}=60)\end{array}$ \\
\hline$<3.0 \mathrm{MET}$ & $365 \pm 100$ & $353 \pm 90$ & $386 \pm 115$ & $391 \pm 94$ & $350 \pm 102^{*}$ \\
\hline 3.0-6.0 MET & $105 \pm 95$ & $123 \pm 106$ & $77 \pm 66^{*}$ & $82 \pm 55$ & $120 \pm 110$ \\
\hline$\geq 6.0 \mathrm{MET}$ & $9 \pm 12$ & $10 \pm 13$ & $9 \pm 11$ & $11 \pm 12$ & $8 \pm 12$ \\
\hline $\begin{array}{l}\text { PAS } \\
\text { (min/day) }\end{array}$ & $\begin{array}{l}\text { Total } \\
(n=101)\end{array}$ & $\begin{array}{l}\text { Men } \\
(n=60)\end{array}$ & $\begin{array}{l}\text { Women } \\
(n=39)\end{array}$ & $\begin{array}{l}B M 1<25 \mathrm{~kg} / \mathrm{m} 2 \\
(n=40)\end{array}$ & $\begin{array}{l}\mathrm{BMI} \geq 25 \mathrm{~kg} / \mathrm{m} 2 \\
(\mathrm{n}=59)\end{array}$ \\
\hline$<3.0 \mathrm{MET}$ & $1241 \pm 134$ & $1220 \pm 158$ & $1277 \pm 72^{* * *}$ & $1243 \pm 114$ & $1242 \pm 146$ \\
\hline 3.0-6.0 MET & $185 \pm 133$ & $206 \pm 155$ & $147 \pm 74^{*}$ & $179 \pm 119$ & $185 \pm 141$ \\
\hline$\geq 6.0 \mathrm{MET}$ & $15 \pm 19$ & $14 \pm 19$ & $16 \pm 19$ & $18 \pm 17$ & $13 \pm 20$ \\
\hline $\begin{array}{l}\text { Accelerometer } \\
\text { (min/day) }\end{array}$ & $\begin{array}{l}\text { Total } \\
(n=43)\end{array}$ & $\begin{array}{l}\text { Men } \\
(n=25)\end{array}$ & $\begin{array}{l}\text { Women } \\
(n=18)\end{array}$ & $\begin{array}{l}B M 1<25 \mathrm{~kg} / \mathrm{m} 2 \\
(n=16)\end{array}$ & $\begin{array}{l}\mathrm{BMI} \geq 25 \mathrm{~kg} / \mathrm{m} 2 \\
(\mathrm{n}=27)\end{array}$ \\
\hline$<3.0 \mathrm{MET}$ & $754 \pm 78$ & $767 \pm 86$ & $737 \pm 63$ & $712 \pm 79$ & $780 \pm 66^{* *}$ \\
\hline 3.0-6.0 MET & $172 \pm 71$ & $165 \pm 82$ & $181 \pm 53$ & $206 \pm 84$ & $151 \pm 54^{*}$ \\
\hline$\geq 6.0 \mathrm{MET}$ & $4 \pm 8$ & $5 \pm 10$ & $2 \pm 3$ & $7 \pm 12$ & $2 \pm 3$ \\
\hline
\end{tabular}

Note. Values are presented as mean $\pm S D$.; comparison of means between gender categories and between BMI categories ${ }^{*} p<.05,{ }^{* *} p<.01,{ }^{* * *} p<.001$. 
According to the PAS women spent significantly more time in light intensity activity than men $(\mathrm{p}<.001)$, and according to both the PAS and SQUASH men spent significantly more time in moderate intensity activity than women $(\mathrm{p}<.05)$. The SQUASH indicated that normal weight individuals spent more time in light activity than overweight individuals ( $\mathrm{p}<.05$ ), while the accelerometer showed normal weight individuals to spend significantly less time in light activity than overweight individuals $(\mathrm{p}<.01)$, but more time in moderate intensity activity $(\mathrm{p}<.05)$.

Comparing minutes of moderate activity per day measured by the SQUASH and accelerometer shows significant differences in the total sample, men, women, normal weight and overweight individuals. The minutes of moderate activity reported by the PAS only differed significantly from the accelerometer in men (Table 2).

Table 2. Minutes of moderate physical activity per day, measured by SQUASH, PAS and accelerometer in 40 respondents.

\begin{tabular}{rlllll}
$\begin{array}{l}\text { Moderate activity } \\
(\mathrm{min} / \text { day })\end{array}$ & $\begin{array}{l}\text { Total } \\
(\mathrm{n}=40)\end{array}$ & $\begin{array}{l}\text { Men } \\
(\mathrm{n}=24)\end{array}$ & $\begin{array}{l}\text { Women } \\
(\mathrm{n}=16)\end{array}$ & $\begin{array}{l}\mathrm{BMI}<25 \mathrm{~kg} / \mathrm{m}^{2} \\
(\mathrm{n}=14)\end{array}$ & $\begin{array}{l}\mathrm{BMI} \geq 25 \mathrm{~kg} / \mathrm{m}^{2} \\
(\mathrm{n}=26)\end{array}$ \\
\hline SQUASH & $89 \pm 69^{* * *}$ & $95 \pm 79^{* *}$ & $79 \pm 52^{* * *}$ & $100 \pm 64^{* *}$ & $83 \pm 73^{* * *}$ \\
\hline PAS & $183 \pm 127$ & $211 \pm 150^{*}$ & $141 \pm 67$ & $213 \pm 161$ & $167 \pm 104$ \\
\hline Accelerometer & $169 \pm 71$ & $163 \pm 83$ & $179 \pm 47$ & $208 \pm 84$ & $149 \pm 53$ \\
\hline
\end{tabular}

Note. Values are presented as mean \pm SD.; comparison of means between SQUASH and accelerometer and between PAS and accelerometer ${ }^{*} p<.05,{ }^{* *} p<.01,{ }^{* * * *} p<.001$.

The correlation between the activity scores of the accelerometer and SQUASH (Fig. 1a) and PAS (Fig. 1b) in the overall sample was only significant between the accelerometer and PAS $(\mathrm{r}=.66$; 95\% C.I. $=.45-.80 ; \mathrm{p}<.001)$. The correlation between the accelerometer and SQUASH was significant for men ( $\mathrm{r}=.62 ; 95 \%$ C.I. $=.29-.82$; $\mathrm{p}<.001)$, but not for women ( $\mathrm{r}=-.49 ; 95 \%$ C.I.=-.80-.01; $\mathrm{p}=.06$ ) (figure $1 \mathrm{c})$. The strength of association between the accelerometer and SQUASH differed significantly between men and women $(\mathrm{p}<.05)$. Although the accelerometer-PAS association was significant for men $(\mathrm{r}=.76$; 95\% C.I. $=.53-.89$; $\mathrm{p}<0.001)$ and nonsignificant for women $(\mathrm{r}=.27$; 95\% C.I. $=-.23-$ .66 ; $\mathrm{p}=.27)$ (figure 1d), the difference in correlations was nonsignificant $(\mathrm{p}=.96)$. The association between the accelerometer and SQUASH activity scores was nonsignificant in normal weight respondents $(\mathrm{r}=.07 ; \mathrm{p}=.81$; 95\% C.I. $=-.48-.58)$, but was significant in overweight respondents ( $\mathrm{r}=.40$; 95\% C.I.=.02-.69; $\mathrm{p}<.05)$ (figure 1e). The accelerometer was significantly associated with the PAS for both normal weight $(\mathrm{r}=.62$; 95\% C.I.=.18-.86; $\mathrm{p}<.05)$ and overweight $(\mathrm{r}=.53 ; 95 \%$ C.I.=.18-.76; $\mathrm{p}<.010)$ respondents (figure 1f) and the difference in association was not statistically significant $(\mathrm{p}=.70)$. 
Figure 1a. Relationship between the accelerometer and SQUASH activity scores ( $\mathrm{r}=.27 ; \mathrm{ns})$ in the total sample $(\mathrm{n}=40)$.

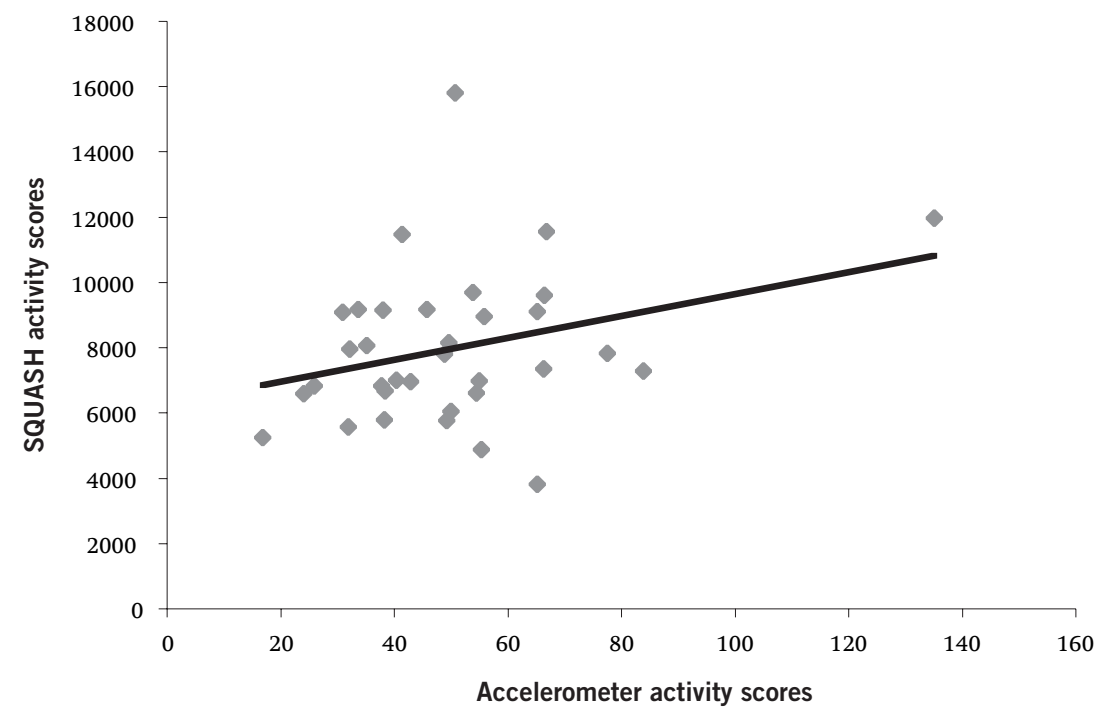

Figure 1b. Relationship between the accelerometer and PAS activity scores $(r=.66 ; p<.001)$ in the total sample $(n=43)$.

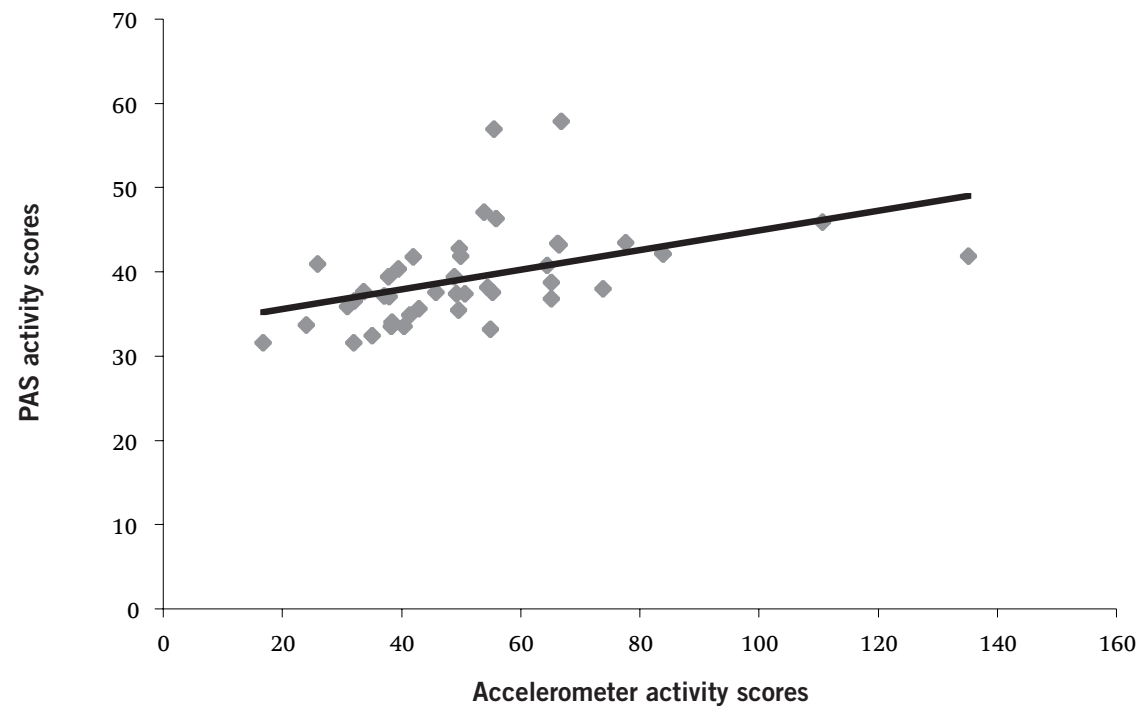


Figure 1c. Relationship between accelerometer and SQUASH in men $(\square n=24, r=.62, p<.005)$ and in women $(\bullet \mathrm{n}=16, \mathrm{r}=-.49, \mathrm{~ns})$.

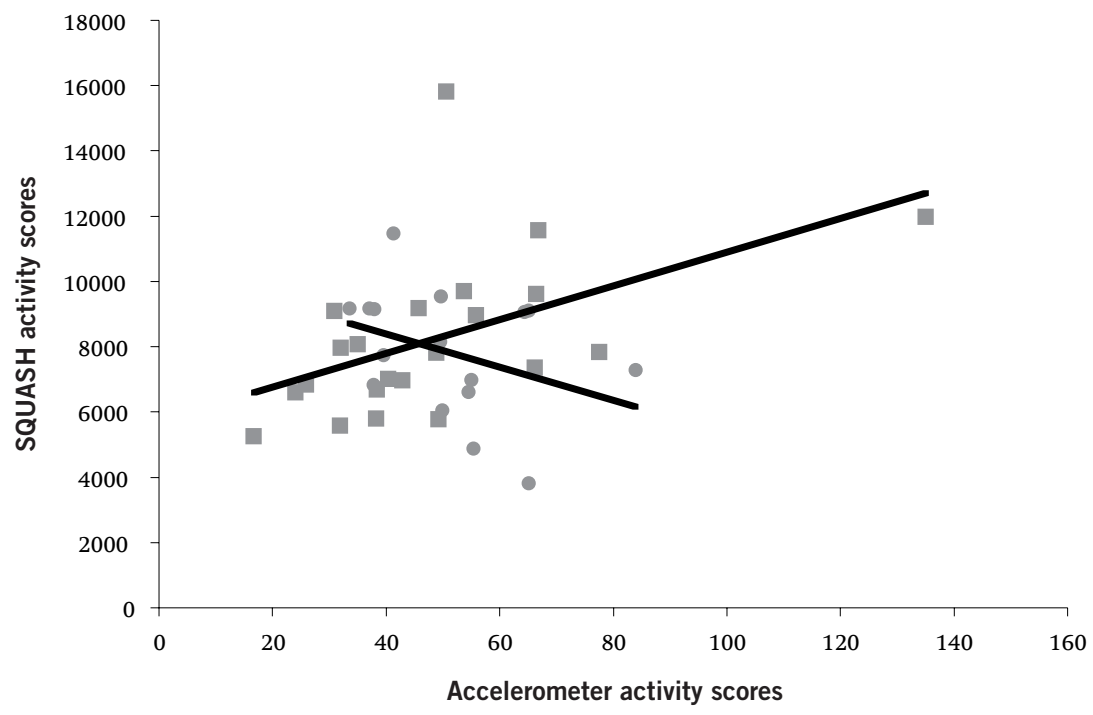

Figure 1d. Relationship between accelerometer and PAS activity scores in men $(\square n=25, r=.76$, $\mathrm{p}<.001)$ and women $(\bullet \mathrm{n}=18, \mathrm{r}=.27, \mathrm{~ns})$.

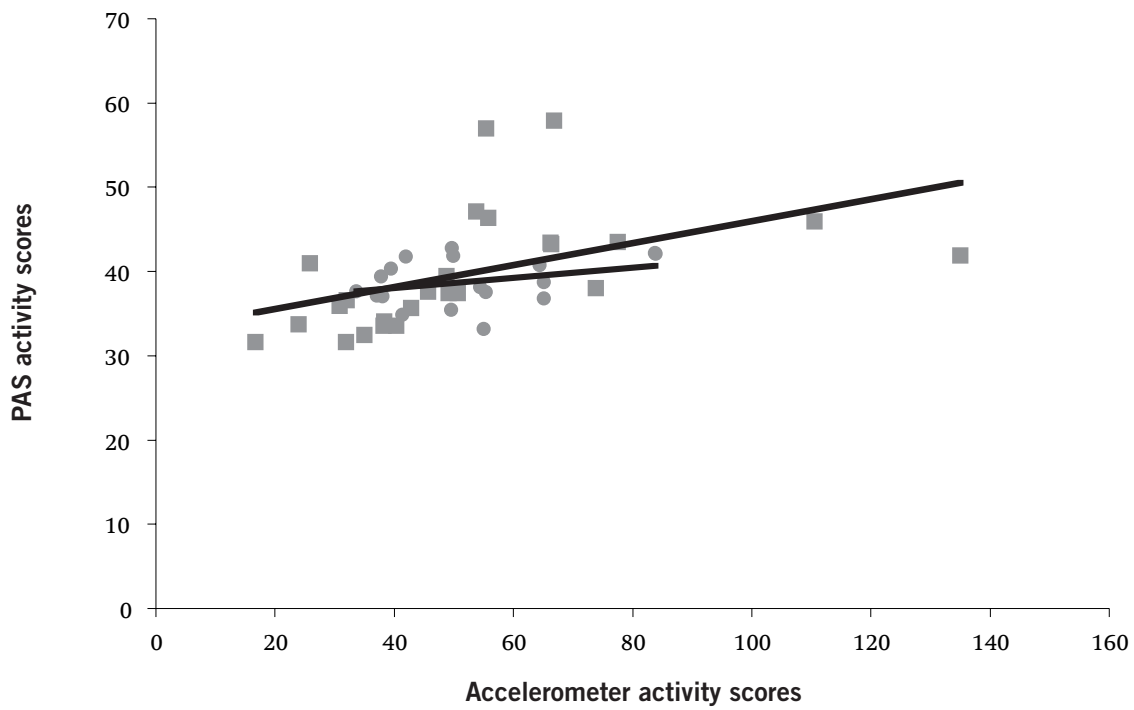


Figure 1e. Relationship between the accelerometer and SQUASH in normal weight $n=14$ $\mathrm{r}=.07, \mathrm{~ns})$ and overweight respondents $(\square \mathrm{n}=26, \mathrm{r}=.40, \mathrm{p}<.05)$.

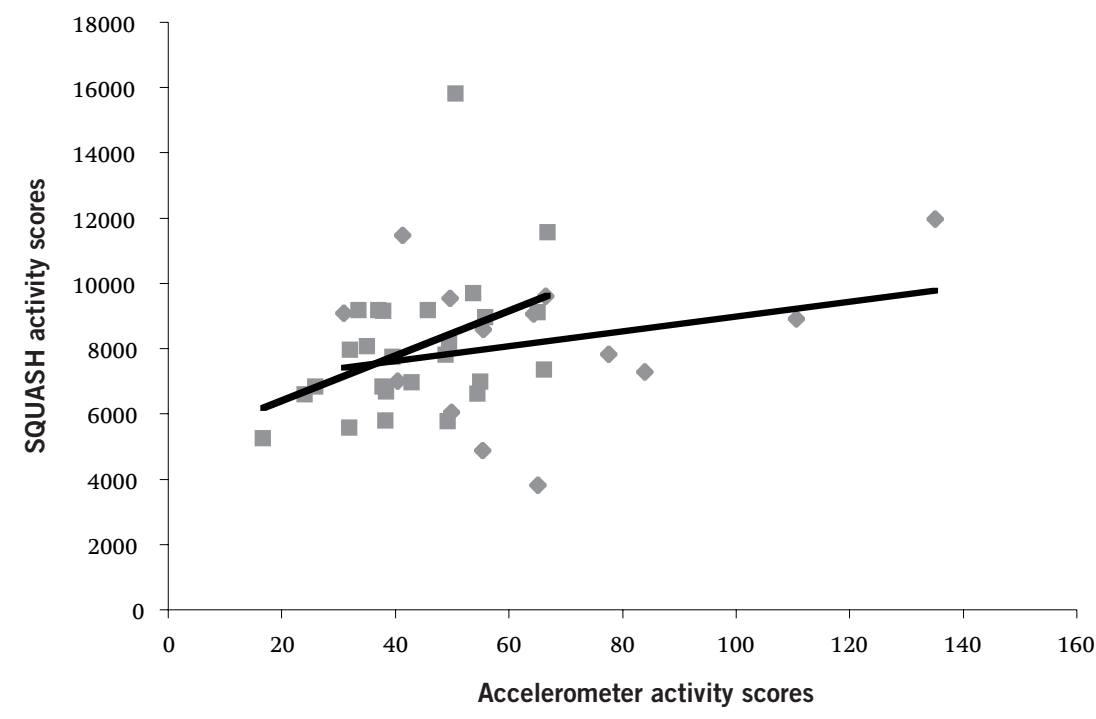

Figure 1f. Relationship between the accelerometer and PAS activity scores in normal weight $(\mathrm{n}=16, \mathrm{r}=.62, \mathrm{p}<.05)$ and in overweight respondents $(\square \mathrm{n}=27, \mathrm{r}=.53, \mathrm{p}<.01)$.

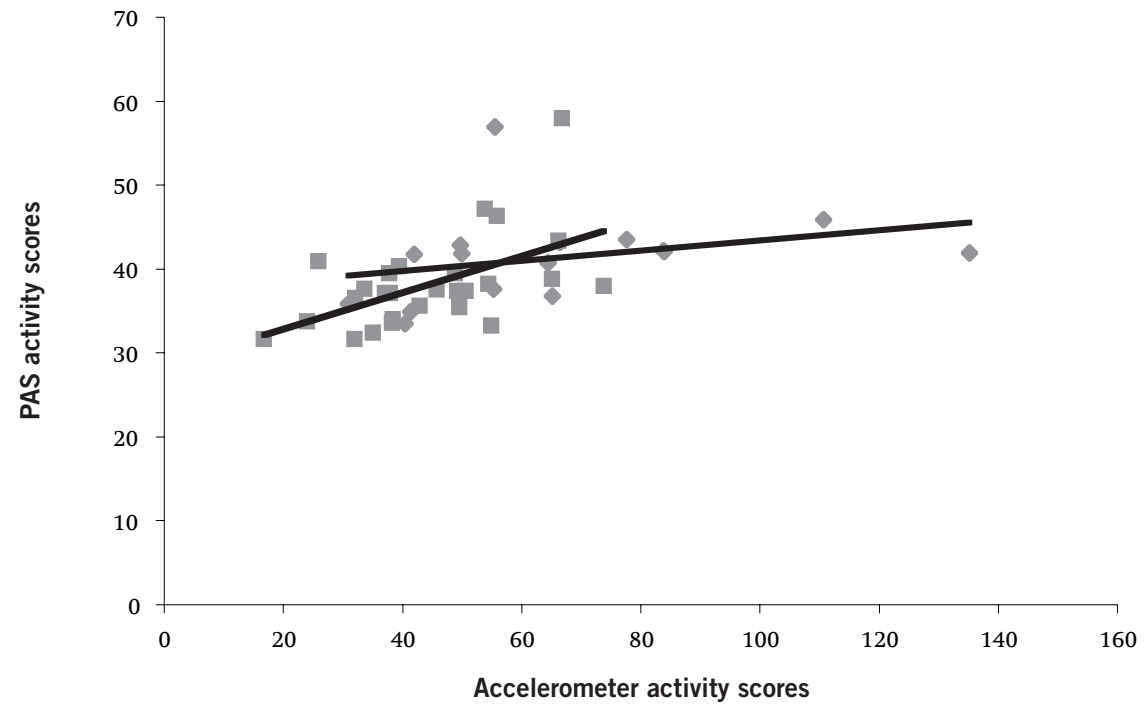

Moderate agreement was found between the tertiles of the accelerometer and PAS activity scores for the overall sample and overweight individuals and substantial agreement was found in men. Agreement between accelerometer and SQUASH did not reach the level of moderate agreement. Fair agreement was found for men (Table 3). 


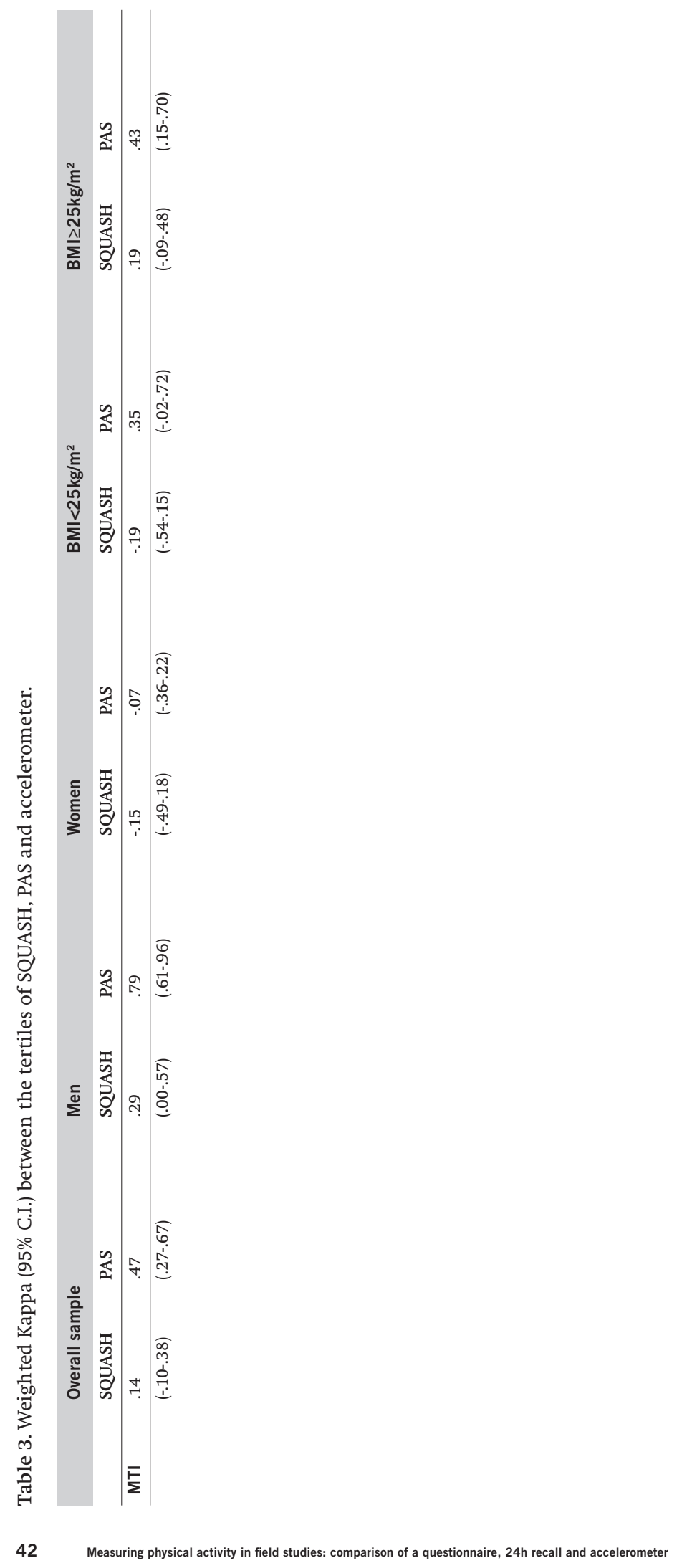




\section{DISCUSSION}

In the present study, a comparison was made between physical activity assessed with a questionnaire, seven $24 \mathrm{~h}$ recalls, and objective data derived from an accelerometer. The PAS correlated significantly with the accelerometer in the overall population, men, normal weight and overweight participants. Correlations ranged from 0.53 to 0.76 . Although the correlation between the PAS and accelerometer was nonsignificant for women, we could not find significantly different performances of the PAS for both sexes. The correlation relating the SQUASH to the accelerometer was only significant for men and overweight individuals. Minutes of moderate activity measured by the accelerometer only differed significantly from that measured by the PAS in men, but differed significantly from that measured by the SQUASH in the total sample and both gender and BMI groups. This discrepancy can be a consequence of questions containing activities with different MET values, but representing a mean MET value. Light cleaning (2.5 METs) for example is classified in the moderate intensity category in the PAS as it is grouped with 3 METs activities, in the SQUASH however it is classified in the light intensity category, as it is not grouped with other activities. In the present study, these analyses were only run for moderate activity since these can be assumed as most accurate, as the SQUASH does not measure activities under 2 METs (Wendel-Vos et al., 2003) and vigorous activity is hardly performed.

This is the first study that used Aadahl's PAS (Aadahl \& Jorgensen, 2003) as a 7-day assessment of physical activity. An earlier study compared a 1-day assessment of the PAS with a physical activity diary and observed a high correlation in the adult sample ( $\mathrm{r}=.74 ; \mathrm{p}<.0001)$ (Aadahl \& Jorgensen, 2003). In the present study, the correlation was higher for men than women. The lack of a significant correlation between the PAS and accelerometer in women could be the consequence of the relatively low percentage of women in our study sample and of the low variance within this population. The relationship between the PAS and accelerometer was not significantly different for men or women, which may indicate that the PAS is equally useful in both sexes. In the present study we further compared the PAS with the accelerometer according to weight status. High correlations were observed both in normal weight and overweight individuals. The SQUASH questionnaire only correlated significantly with the accelerometer in men. This correlation was higher than was observed in an earlier study $(\mathrm{r}=.45 ; \mathrm{p}<.05)$, in which the SQUASH was also compared with an accelerometer. The authors concluded that the relative validity of the SQUASH to assess physical activity in the general population was within the upper range compared to other questionnaires (Wendel-Vos et al., 2003). The present study can partly support these findings for males. Both concerning the PAS and SQUASH we reported discrepancies in the correlations between men and women. As has been suggested before (Richardson et al., 2001) completion of questionnaires like SQUASH may be gender-dependent. One explanation is that in general men report more high intensity activities than women, which are less prone to measurement error than lower intensity activities (Durante \& Ainsworth, 1996). A greater percentage of the high intensity activity performed by men involves well-defined activities, such as sports, which are more accurate to recall than the usually non-sport or exercise related high intensity activities performed by women (Richardson et al., 2001).

In general, the PAS appeared to correlate better with the accelerometer than the SQUASH. The discrepancy between the PAS and SQUASH could be a consequence of the way both self-measures are structured and of the way they are implemented. The PAS consists of ten categories of which four categories contain activities with a MET level between 0.9 and 2.0 METs, such as watching television, lis- 
tening to music and eating. The SQUASH however only contains activities above 2.0 METs. As the study population consisted of relatively inactive subjects (according to the accelerometer measurements the overall population spent $98 \%$ of total activity in the light activity category), listing these light activities in a questionnaire that assesses physical activity is highly relevant. Especially when keeping in mind that the general population becomes more sedentary, only listing activities with a MET above 2.0 METs, could influence the validity of the questionnaire. The discrepancy in implementation is related to the fact that with regard to the PAS respondents are asked to complete it daily for one week, with the risk of hitting an atypical week, for the SQUASH they are asked to refer to a typical week. Furthermore the PAS is less subject to errors due to restrictions imposed by memory (Chasan-Taber et al., 2002).

There are methodological aspects of this study that need to be considered. The first relates to the use of accelerometers as comparison. Validation of the MTI Actigraph accelerometer against the DLW method showed correlations of 0.41 to 0.67 (Leenders et al., 2006), but validation studies have also shown that the MTI underestimates energy expenditure in older men (Starling et al., 1999) and younger females (Leenders et al., 2001) and overestimates expenditure in obese women (Fogelholm et al., 1998). Despite these discrepancies, accelerometers are suggested to be a valuable tool for comparing activity levels between groups of individuals (Bassett et al., 2000). However, accelerometers do not register all activities, such as arm movements and activities like cycling, stair climbing or weight lifting accurately (Bassett et al., 2000), which may lead to a possible underestimation of physical activity levels. A second limitation pertains to the large dropout as a consequence of individuals not wearing the accelerometer correctly. This was mainly due to inconvenience, which might imply that the accelerometer is not very user friendly and consequently a less useful measure of total physical activity. In addition, the present study used a relatively small research population with a small range in age, weight and physical activity, which makes extrapolation to a larger population more difficult. A fourth limitation concerns the comparison within the gender and BMI groups. As groups were not matched, caution is warranted for the interpretation of these results. A final limitation is the lack of consensus regarding the most appropriate regression equation to accurately transform activity counts into MET values and more specifically thecut-pointestimates thatareused toclassifyactivitiesintointensityranges (Bassett et al., 2000; Hendelman et al., 2000). In the present study the regression model of Swartz et al. (2000) was chosen, as this equation was not solely derived from laboratory-based locomotor activities as many other equations, but also from free-living activities (Swartz et al., 2000). Additionally, research by Strath and colleagues (2003) has shown that estimates from Swartz cut-off points for estimating time spent in the different intensity activity categories were not different from the criterion measure on group basis (Strath et al., 2003).

Despite these limitations, it can be concluded that a 7-day administration of the PAS is a useful measure for level and classification of physical activity, in this specified population. The SQUASH has practical advantages in terms of convenience of administrating, but its usefulness in estimating PA levels is limited. 


\section{CONCLUSION}

This study provides important practical information regarding the use of physical activity measures in large-scale studies. Although physical activity questionnaires do have practical value in monitoring changes in population activity, a recent review suggested that standardized self-report instruments should be developed that record the low intensity activities that are so typical of sedentary societies (Shephard, 2003). The results of the present study suggest that the PAS is an instrument that may meet this requirement. Large-scale studies could use the 7-day administration of the PAS in the overall sample, combined with an objective measure for a subsample in order to provide indications on its validity in that specific study (Kremers et al., 2005d). Only when a 7-day administration of the PAS could be regarded as too intrusive for study participants, researchers could decide to use a frequency questionnaire, such as the SQUASH as a measure of physical activity, however taking into consideration the gender-dependency and relatively limited value of such a measure to provide accurate information on actual physical activity levels. 


\section{CHAPTER 4}

\section{FORMATION OF IMPLEMENTATION INTENTIONS PROMOTES STAIR USE}

Based on paper published as: Kwak, L., Kremers, S.P.J., Van Baak, M.A. \& Brug, J. (2007). Formation of implementation intentions promotes stair use. American Journal of Preventive Medicine, 32, 254-255 


\section{ABSTRACT}

Stair use has been identified as a useful behaviour for lifestyle exercise interventions. The present study investigated whether forming an implementation intention specifying stair use is effective in stimulating this behaviour. Participants (143 employees of an academic hospital) underwent anthropometric measurements and were asked to form an implementation intention specifying either stair use (intervention condition) or cycling in leisure time (control condition). Once participants had formulated their implementation intention, a researcher, who was inconspicuously stationed in the hallway near the measuring room, observed whether participants took the stairs or elevator directly after leaving the examination room. Of the intervention condition $83.3 \%$ used the stairs after leaving the examination room in comparison to $66.7 \%$ of the control condition. Stair use was influenced by a positive attitude toward stair use as a means to watch one's weight and a lower BMI $\left(\mathrm{kg} / \mathrm{m}^{2}\right)$. Forming an implementation intention specifying stair use had an additional effect.

It can be concluded that short-term stair use can be positively influenced by a short intervention using the formation of implementation intentions. 


\section{INTRODUCTION}

Physical inactivity is a major risk factor for coronary heart disease, type 2 diabetes, hypertension and colon cancer (Dunn et al., 1999; Stephenson et al., 2000). Promoting physical activity can thus be regarded as a public health priority. Attempts have conventionally focussed on structured exercise programmes, but with limited evidence of success (Kahn et al., 2002; Hillsdon et al., 2005). An alternative may be a lifestyle approach to physical activity, which emphasizes on seeking opportunities in one's lifestyle to engage in physical activity (Jakicic \& Otto, 2005). The environment provides many of these opportunities, giving individuals the choice for either active or sedentary behaviours (Foster \& Hillsdon, 2004), such as the provision of both stairs and elevators. As opportunities for stair use are frequently available and no costs are involved, stair use has been identified as a useful model for lifestyle exercise interventions (Kerr et al., 2001a). Stimulating stair use coincides with preventing or reducing the undesirable habitual behaviour of elevator use. Habits are automatic behavioural routines elicited in the same environment; changing the environment may obstruct the automatic link (Kerr et al., 2001a). The formation of implementation intentions has proven a useful strategy to do this (Gollwitzer, 1999; Sheeran et al., 2005a). An implementation intention is a specific action plan concerning exactly how, when and where an intended goal-directed behaviour will be performed. It has the structure of "When situation $\mathrm{x}$ arises, I will perform response y!" and links the anticipated situational context with goal-directed behaviours (Gollwitzer \& Oettingen, 1998; Gollwitzer, 1999). The present study investigated whether an intervention using implementation intentions is effective in stimulating stair use in a worksite setting.

\section{METHODS}

The sample (143 academic hospital employees; $85 \%$ female, mean age 38.4yr) contained participants of a worksite-based weight gain prevention programme (NHFNRG In Balance-project; for more information, see Kremers et al., 2005a), which included individual and environmental interventions. Annually participants underwent anthropometric measurements (height $(\mathrm{m})$ and weight $(\mathrm{kg})$ ) in an examination room in the basement of the hospital and completed a questionnaire. The questionnaire included items regarding gender, attitude toward stair use as a means to watch one's weight ('For me, taking the stairs more often is an appropriate method to watch my weight'; 5-point scale: strongly agree (+2) - strongly disagree (-2)), intention to be more physically active at work ('Are you planning to be more physically active at work the coming 6 months?'; 5-point scale: definitely (+2) -definitely not (-2)) and past behaviour regarding stair use ('How often do you take the stairs per day?'; open-ended item).

Following the measurements of 2005, participants were asked to form an implementation intention. They received a sheet of paper containing the following text: "People who want to move more, can do so in different ways. For example by exercising more, cycling in leisure time or by taking the stairs instead of the elevator. People who have specified as precisely as possible how they are going to change their physical activity have the highest rate of success". Accordingly, half of the participants (randomly allocated) were given an example of a specific action plan regarding taking the stairs instead of the elevator (intervention condition). They were then asked to indicate their habitual stair use (5-point scale: 0 (I always take the elevator) - 5 (I always take the stairs)) and instructed to form an implementation 
intention specifying when and where at work they would take the stairs instead of the elevator. Two lines were provided to write down the precise timing and location for doing so. The instructions given to the other half of the participants (control condition) were identical in all aspects apart from the goal behaviour, which was cycling in leisure time.

Once participants had formulated their implementation intention, a second researcher, who was inconspicuously stationed in the hallway of the examination room, observed whether participants took the stairs or elevator (adjacent) directly after leaving the examination room. The observer was blinded to the participants' assignment to a condition.

\section{Data analysis}

Data were analyzed in 2005/2006 using SPSS 11.5 statistical package. To ensure successful randomization multivariate analyses were conducted to compare both groups with respect to gender, body mass index (BMI; calculated as weight (kg) divided by height $(\mathrm{m})$ squared $\left.\left(\mathrm{kg} / \mathrm{m}^{2}\right)\right)$, past behaviour, attitude and intention. Logistic regressions were executed with stair use as dichotomous dependent variable and age, gender, BMI, attitude, intention, past behaviour and condition as independent variables. Interactions between condition and the other variables were explored.

\section{RESULTS}

There was a loss to follow-up of 39\%, a result of the proportion of hospital employees remaining on the same floor; their stair use could therefore not be observed. An intention to treat analysis, however, showed similar results as those presented below. Stair use was monitored of 87 participants (42 intervention and 45 control condition). The intervention and control conditions did not differ in terms of gender $(\mathrm{OR}=.37$; 95\% C.I. $=.10-1.37$, ns), BMI (OR=1.05; 95\% C.I.=.93-1.20, ns), attitude $(\mathrm{OR}=1.00$; 95\% C.I. $=.61-1.63$, ns), intention $(\mathrm{OR}=1.11$; 95\% C.I.=.67-1.87, ns) and past behaviour $(\mathrm{OR}=1.04 ; 95 \%$ C.I. $=.98-1.10$, ns).

Of the intervention condition $83.3 \%$ used the stairs after leaving the examination room in comparison to $66.7 \%$ of the control condition. Stair use was influenced by a positive attitude toward stair use as a means to watch one's weight and a lower BMI (Table 1). Forming an implementation intention specifying stair use had an additional effect. There were no significant interactions between condition and any of the other variables.

Table 1. Results of the logistic regression analyses with stair use as dependent variable.

\begin{tabular}{lll} 
& OR & $95 \%$ C.I. \\
Age & 1.00 & $.93-1.07$ \\
\hline Gender & .38 & $.07-2.25$ \\
\hline BMI & $.85^{*}$ & $.73-.99$ \\
\hline Attitude & $2.01^{*}$ & $1.07-3.77$ \\
\hline Intention & .78 & $.40-1.51$ \\
\hline Past behaviour & .96 & $.91-1.02$ \\
\hline Condition & $3.52^{*}$ & $1.09-11.36$ \\
\hline
\end{tabular}

Note. ${ }^{*} p<.05$. 


\section{DISCUSSION}

This study showed that individuals who specified when and where they would take the stairs were more likely to perform this behaviour than those who had formed an unrelated implementation intention. To our knowledge, this is the first study that has investigated the effect of implementation intentions on stair use. Interventions directed at stimulating stair use are often moderated by demographic variables, such as gender and BMI (Andersen et al., 1998; Russell et al., 1999; Coleman \& Gonzalez, 2001; Kerr et al., 2001a). However, in the present study neither gender nor BMI played a moderating role in the effect of the intervention. A main effect of BMI was identified, indicating more stair use for those with a lower BMI. Additionally, stair use was predicted by the belief that stair use is an adequate means to watch one's weight. This corresponds with findings of Sheeran and Orbell (2000), who concluded that behaviour-specific cognitions provided good prediction of attendance to cervical cancer screening and the inclusion of the implementation intention condition as a predictor further enhanced the prediction of attendance behaviour (Sheeran \& Orbell, 2000). This study has several limitations. First, the hospital and participants were part of a larger study (Kremers et al., 2005a). The results can therefore not simply be generalized to other settings or persons. Participants were representative for the population in terms of prevalence of overweight $(35 \%)$, though. Second, participants were only observed directly after leaving the examination room. It would be interesting to perform a longer follow-up study, especially as it has been suggested that implementation intentions can be seen as a valuable supplement to motivational interventions as they contribute to increased likelihood of action in the longer term (Sheeran \& Orbell, 1999).

The present study is promising for future lifestyle physical activity interventions, as stair use was stimulated by means of a simple and inexpensive intervention. It did however still require the presence of an instructor. It would be relevant to investigate if the manipulation would also lead to an increase in stair use if the manipulation was more self-regulatory, for example by e-mail. 



\section{CHAPTER 5}

\section{LONG-TERM EFFECTIVENESS OF IMPLEMENTATION INTENTIONS REGARDING STAIR USE: A 12 MONTHS FOLLOW-UP}

Resubmitted (minor revisions): Kwak, L., Kremers S.P.J., Van Baak, M.A. \& Brug, J. Long-term effectiveness of implementation intentions regarding stair use: a 12 months follow-up 


\section{ABSTRACT}

This study evaluates the effectiveness of implementation intentions on stair use over a 12-month period in a worksite setting. At baseline 168 academic hospital employees, underwent anthropometric measurements in an examination room in the basement of the hospital, completed a questionnaire that included items regarding age, gender, attitude, intention and past behaviour regarding stair use and formed an implementation intention specifying either stair use (intervention condition) or cycling in leisure time (control condition). Twelve months later participants returned to the same examination room and reported if they had taken the stairs or elevator to the basement. Individuals in the intervention condition were more likely to take the stairs 12 months later than control individuals, however only among individuals who were motivated at baseline to be more active at work. This is the first study that has observed a significant effect of implementation intentions over a period of 12 months. 


\section{INTRODUCTION}

Various studies have demonstrated that health behaviour change goals are more likely to be achieved when they are furnished with implementation intentions (Gollwitzer \& Sheeran, 2006). According to Gollwitzer (1990) there are two action phases preceding the occurrence of behaviour, namely a motivational phase that should result in making intentions to engage in a behaviour, and a subsequent volitional phase during which people should act according to their goal intentions (Gollwitzer, 1990). Implementation intentions have been found to be important during this volitional phase, by specifying exactly when, where and how these behaviours are to be achieved (Gollwitzer, 1993, 1996, 1999), thereby linking an anticipated future situational cue with a certain goal-directed behaviour (Gollwitzer \& Oettingen, 1998). The formation of an implementation intention thus delegates the control of one's behaviour from the self to the specified situational cue (Gollwitzer, 1999). It is assumed that when the anticipated situational cue is encountered this leads to the automatic activation of the intended goal-directed behaviour (Gollwitzer, 1999), which is elicited immediately, efficiently and without conscious intent (Brandstatter, 2001).

The assumption that implementation intentions involve automatic links between cues in the environment and behaviours that are associated with these links (Verplanken \& Faes, 1999), suggests that implementation intentions should maintain their effectiveness over time whenever the anticipated cue is actually encountered. However, sustainability of effects of implementation intentions has rarely been tested (Prestwich et al., 2005). To date only three studies (Jackson et al., 2005; Prestwich et al., 2005; Sheeran \& Orbell, 2000) have assessed the effectiveness of implementation intentions over a period of more than two months, of which two studies observed significant effects (Prestwich et al., 2005; Sheeran \& Orbell, 2000). Sheeran and Orbell (2000) found that participants who formed implementation intentions were more likely to attend cervical screening during a 3-month period compared with controls. Prestwich and colleagues (2005) showed that implementation intentions were effective in increasing the likelihood of breast self-examination performance both at one and six months. Additionally, Ziegelmann and colleagues (2006) investigated how action planning, a closely related concept to implementation intentions (Leventhal et al., 1965; Lippke et al., 2004), and coping planning support the adoption and maintenance of physical activity after a six-month follow-up and found action plans to be predictive of long-term maintenance at six month (Ziegelmann et al., 2006). Long-term studies are needed to further assess the effectiveness of implementation intentions over longer periods of time.

Additionally, studies are needed that investigate the beneficial impact of implementation intentions on goal achievement in real-life settings. A recent meta-analysis of 94 independent implementation intention studies revealed that most studies were conducted among university students (82.3\%) and only $8.3 \%$ sampled individuals of the general public. The rest of the samples contained either individuals with physical illnesses or psychological problems $(7.3 \%)$ or children/ young people (2.1\%) (Gollwitzer \& Sheeran, 2006).

The purpose of the present study was to test the long-term effectiveness of the formation of implementation intentions in a real-life setting. It proceeds on a previous study (Kwak et al., 2007a), which showed the effectiveness of implementation intentions regarding stair use in a worksite setting, based on independent observation of the goal behaviour. Individuals who specified when and where they would take the stairs at work were more likely to perform 
this behaviour immediately after forming an implementation intention than those who had formed an unrelated implementation intention. The present study tested whether effects of those implementation intentions were maintained after 12 months.

\section{METHODS}

\section{Participants and Procedure}

Participants were 168 academic hospital employees, who took part in a worksite-based weight gain prevention programme, which included individual and environmental interventions (NHF-NRG In Balance-project, see Kwak et al., 2007b). The present study involved two time-points (baseline and 12 months follow-up). At baseline participants underwent their annual anthropometric measurements (height $(\mathrm{m})$ and weight $(\mathrm{kg})$ ) in an examination room in the basement of the hospital and completed a questionnaire. The questionnaire included items regarding age, gender, attitude toward stair use as a means to watch one's weight ('For me, taking the stairs more often is an appropriate method to watch my weight'; 5-point scale: strongly agree $(+2)$ - strongly disagree $(-2)$ ), intention to be more physically active at work ('Are you planning to be more physically active at work the coming 6 months?'; 5-point scale: definitely (+2) -definitely not (-2)) and past behaviour regarding stair use ('How often do you take the stairs per day?'; open-ended item).

Following the anthropometric measurements participants were asked to form an implementation intention. They received a sheet of paper containing the following text: "People who want to be more physically active, can do so in different ways. For example by exercising more, cycling in leisure time or by taking the stairs instead of the elevator. People who have specified as precisely as possible how they are going to change their physical activity have the highest rate of success". Half of the participants (randomly allocated) were subsequently given an example of a specific action plan regarding taking the stairs instead of the elevator (intervention group). They were instructed to form an implementation intention specifying when and where at work they would take the stairs instead of the elevator. Two lines were provided to write down the precise timing and location for doing so. The instructions given to the other half of the participants (control group) were identical in all aspects apart from the goal behaviour, which was cycling in leisure time. After the participants left the examination room, an inconspicuously stationed observer situated at the ground floor monitored their elevator or stair use. These observations showed that participants who made implementation intentions for stair use were 3.5 times more likely to take the stairs after the examination (Kwak et al., 2007a). Twelve months later participants returned to the same examination room at the basement of the hospital and were asked among other things if they had taken the stairs or elevator to the basement.

\section{Statistical analysis}

Data were analyzed in 2005/2006 using SPSS 11.5 statistical package. T-tests and chisquare tests were conducted to compare both groups with respect to gender, body mass index (BMI; calculated as weight $(\mathrm{kg})$ divided by height $(\mathrm{m})$ squared $\left(\mathrm{kg} / \mathrm{m}^{2}\right)$ ), past behaviour, attitude and intention. To examine whether there was a significant difference in the proportion of individuals taking the stairs in the control and intervention group, a logistic regression was conducted with stair use as dichotomous dependent variable and age, gender (male (0), female(1)), BMI, attitude (negative (-2) - positive (+2)), 
intention (definitely not (-2) - definitely yes (+2)), past behaviour and control/intervention group (control (0), intervention (1)) as independent variables. Interactions between control/intervention group and the other variables were also explored. All p-values are two-sided and 5\% level of significance was used for all analyses.

\section{RESULTS}

\section{Representativeness and Randomization Checks}

In total 137 participants of the academic hospital (715 beds; 5 floors) were present at both time-points (81.6\%). Occupational categories of the participants included medical staff, technicians, laboratory workers, kitchen personal and individuals from economy departments. An intention to treat analysis showed similar results as those presented below. Twenty-five participants were removed from the sample as they had indicated that they had come from the same floor as the examination room, so did not take the stairs or elevator. This left 112 participants, 58 (51.8\%) in the control group and $54(48.2 \%)$ in the intervention group. Seventy-one percent of participants were female and the mean age was 39.6 years $(\mathrm{SD}=7.8)$. The intervention and control groups did not differ significantly in terms of age, gender, BMI, past behaviour, attitude and intention (Table 1), although the difference in past behaviour approached significance.

Table 1. Descriptives of intervention and control group.

\begin{tabular}{llll} 
& $\begin{array}{l}\text { Intervention }(\mathrm{n}=54) \\
\text { mean } \pm \text { SD }\end{array}$ & $\begin{array}{l}\text { Control }(\mathrm{n}=58) \\
\text { mean } \pm \text { SD }\end{array}$ & $\begin{array}{l}\text { Test of significance } \\
(\mathrm{p} \text {-value })\end{array}$ \\
\hline Age, $\mathrm{y}$ & $39.5 \pm 8.3$ & $39.7 \pm 7.4$ & $\mathrm{t}=0.15(.88)$ \\
\hline Gender, \% women & 64.8 & 77.6 & $\mathrm{X}^{2}=2.24(.14)$ \\
\hline BMI, $\mathrm{kg} / \mathrm{m}^{2}$ & $24.3 \pm 2.9$ & $24.6 \pm 3.8$ & $\mathrm{t}=0.49(.63)$ \\
\hline Attitude +2 to -2$)$ & $0.71 \pm 0.9$ & $0.77 \pm 0.7$ & $\mathrm{t}=0.40(.69)$ \\
\hline Intention $(+2$ to -2$)$ & $-0.20 \pm 0.9$ & $-0.19 \pm 0.9$ & $\mathrm{t}=0.04(.97)$ \\
\hline $\begin{array}{l}\text { Past behaviour, } \\
\text { number of stairs }\end{array}$ & $9.2 \pm 9.4$ & & $\mathrm{t}=-1.95(.05)$ \\
\hline
\end{tabular}

\section{Effects of the Intervention}

The results of the logistic regression analyses revealed that in the intervention group $83.3 \%$ of participants reported to have taken the stairs compared to $75.9 \%$ of the control group ( $\mathrm{OR}=1.51$; ns; Table 2), with heavier individuals being less inclined to take the stairs.

A significant interaction $(\mathrm{B}=14.09, \mathrm{p}<.01)$ was observed between control/ intervention group and the intention to be more physically active at work, which led to a stratification of the group into individuals with a low intention to be more physically active at work $(<0 ; n=55)$ and those with a high intention $(\geq 0 ; n=57)$. Forming an implementation intention specifying stair use only had a significant effect on stair use if individuals had a high intention to be more physically active at work (Table 3). 
Table 2. Results of the logistic regression analysis with stair use as dependent variable.

\begin{tabular}{lcc} 
& OR & $95 \%$ C.I. \\
Age & 1.03 & $.97-1.10$ \\
\hline Gender, men (0)-females (1) & .65 & $.21-2.02$ \\
\hline BMI, kg/m & $.86^{*}$ & $.75-.99$ \\
\hline Attitude, very negative (-2)- very positive (+2) & 1.46 & $.79-2.71$ \\
\hline Past behaviour, number of stairs & 1.01 & $.93-1.09$ \\
\hline Intention, definitely not (-2)- definitely yes (+2) & 1.01 & $.58-1.77$ \\
\hline Intervention/control group, control (0)-intervention (1) & 1.51 & $.56-4.09$ \\
\hline
\end{tabular}

Note. ${ }^{*} p<.05$.

Table 3. Results of the logistic regression analyses with stair use as dependent variable in individuals with a low-and high intensity to be more physically active at work.

\begin{tabular}{lllll} 
& \multicolumn{2}{l}{ Low intention $(<0 ; \mathrm{n}=55)$} & \multicolumn{2}{l}{ High intention $(\geq 0 ; \mathrm{n}=57)$} \\
& OR & $\mathbf{9 5 \%}$ C.I. & OR & 95\% C.I. \\
\hline Age & 1.01 & $.92-1.12$ & 1.07 & $.98-1.16$ \\
\hline Gender & .50 & $.08-3.22$ & .58 & $.13-2.70$ \\
\hline BMI & .83 & $.66-1.03$ & .85 & $.68-1.06$ \\
\hline Attitude & 1.01 & $.39-2.57$ & 2.61 & $.96-7.09$ \\
\hline Past behaviour & 1.07 & $.89-1.28$ & .98 & $.90-1.07$ \\
\hline Condition & .31 & $.06-1.62$ & $7.87^{*}$ & $1.09-44.59$ \\
\hline
\end{tabular}

Note. ${ }^{*} p<.05$.

\section{DISCUSSION}

Among individuals who were motivated to be more active at work we demonstrated that those, who formed an implementation intention regarding taking the stairs instead of the elevator, were more likely to perform this behaviour 12 months later than individuals who performed an unrelated implementation intention. This is the first study that has observed a significant effect of implementation intentions over a period of 12 months. The results give further support to the belief that the mental link that is created between a behaviour and certain contextual cues, as a result of forming an implementation intention, produces such strong memory traces that encountering the relevant contextual cues results in performance of the specified behaviour (Sheeran \& Orbell, 1999). The effectiveness of implementation intentions in the present study was dependent on the presence of positive goal intentions, which corresponds with previous findings (Sheeran et al., 2005b). As suggested by Sheeran and colleagues this supports the idea that although action control by implementation intentions exhibits features of automatic processes such as immediacy and efficiency, these effects are conditional on the presence of relevant goal intentions (Sheeran et al., 2005a). In this sense, implementation intentions possess a mix of automatic and controlled features, also referred to as strategic automaticity (Bargh \& Gollwitzer, 1994; Gollwizter, 1999). Interestingly, data collected immediately after formation of the implementation intention, revealed that the effectiveness of the implementation intentions was not dependent on the presence of strong goal intentions (Kwak et al., 2007a). This would imply that there might be a different mechanism behind the effect of 
implementation intention regarding action initiation and action maintenance. Ziegelmann and colleagues (2006) provided evidence for differential prediction patterns in adoption and maintenance of behaviour and found action plans to be of importance both with regard to initiation and maintenance. With regard to the present study, recently formed implementation intentions may be prompted by situational cues, leading to the performance of the specified behaviour even among individuals who are not motivated to change. However, motivation to change may be a prerequisite for implementation intentions to remain their effectiveness over time.

The present study has several strengths. It is the first study to show effects of implementation intentions at 12-month follow-up, and it is one of only a few studies to test implementation intentions in a non-student sample (Gollwizter \& Sheeran, 2006).

A number of potential limitations of the present study should be noted. The first limitation concerns the self-reported measure of behaviour. However, given that the behavioural assessment was embedded within a wide range of questions asked when anthropometric measurements were performed, it seems unlikely that answers were influenced by social desirability. A second limitation pertains to the difference in stair use at baseline between the intervention and control group, which approached statistical significance, even though a completely random treatment approach was applied. Our logistic regression analyses however showed that this particular measure (i.e. 'past behavior, number of stairs') was not a significant predictor of stair use, implying that the results were in fact controlled for baseline behaviour, and that the difference in past behaviour most likely has not influenced the present study findings. A third limitation pertains to the research population; both the hospital and participants were part of a larger study (Kwak et al., 2007b). The observed results can therefore not simply be generalized to all kind of settings or persons. Participants seemed to be a representative sample of the population regarding variables such as gender, age and BMI, though. Additionally, results can not simply be generalized to overall stair use, as participants only reported their stair use to the anthropometric assessment. However, implementation intentions were formed with regard to overall stair use at work. A fourth limitation concerns the small sample sizes of both intention groups after stratification, resulting in small numbers of participants taking the elevator, especially in the high intention group, which led to a large confidence interval of the implementation intention effect size. Caution is warranted for the interpretation of the Odds Ratios. Given the large confidence intervals in some instances, the estimate of the effect size could be imprecise. Finally, this study could not establish whether implementation intentions resulted in an increase in total physical activity, which is relevant for public health.

Notwithstanding the potential limitations of the present study, there are also several potential practical applications. Firstly, our results support the use of implementation intention with regard to maintenance of health behaviours, which is essential as health benefits often only emerge from health behaviours when they are sustained over time. Additionally, our results confirm that implementation intentions are most effective at changing behaviour on the long-term when they are targeted at people with a positive intention to change their behaviour, which indicates that both motivational and volitional interventions are necessary (Dholakia \& Bagozzi, 2003; Milne et al., 2002; Orbell et al., 1997; Prestwich et al., 2003) 



\section{CHAPTER 6}

\section{A POSTER-BASED INTERVENTION TO PROMOTE STAIR USE IN BLUE- AND WHITE-COLLAR WORKSITES}

Paper published as: Kwak, L., Kremers, S.P.J., Van Baak, M.A. \& Brug, J. (2007) A poster-based intervention to promote stair use in blue-and white-collar worksites. Preventive Medicine, 45, 177-181 


\section{ABSTRACT}

Previous studies have generally shown the effectiveness of prompts to promote stair use in worksites that mainly consist of white-collar workers. The present study tested whether an intervention using prompts, is effective in stimulating stair use in two types of worksites; one consisting mainly of white-collar workers and one mainly of blue-collar workers. In 2005, elevator and stair use (stair climbing and descent) was monitored in two types of worksites in the Netherlands, namely one office building ( $\mathrm{n}=150$ white-collar workers) and one paper factory ( $\mathrm{n}=800$ blue-collar workers). The study used a simple time-series design of collecting data in three waves: before, during and after implementation of posters containing prompts stimulating stair use. A total of 6771 choices between stairs and elevator were observed. There was a significant difference between stair use at baseline and during the poster intervention in both types of worksites. There was no worksite-by-intervention interaction, implying that the prompts were equally effective in both types of worksites. After removal of the posters stair use decreased significantly to a level that was not significantly different from baseline. In conclusion, stair use can be positively influenced in both blue- and white-collar workers by a short-term low-cost intervention using prompts on posters. 


\section{INTRODUCTION}

Regular stair use can have important implications for public health, by improving cardiovascular fitness (Boreham et al., 2005; Teh \& Aziz, 2001), reducing cholesterol levels (Boreham et al., 2000) and by contributing to weight control (Eves et al., 2006b). As stair use opportunities are frequently available and do not involve any costs, it is a useful strategy for lifestyle physical activity interventions (Kerr et al., 2001a). To date all published studies that have attempted to increase stair use by using poster prompts in commercial settings, such as bus stations, malls and airports have been successful (Andersen et al., 1998, 2000; Blamey et al., 1995; Brownell et al., 1980; Coleman \& Gonzalez, 2001; Kerr et al., 2000, 2001a, b, c, d; Russell \& Hutchinson, 2000; Webb \& Eves, 2005).

Prompts are relatively short motivating messages, which refer to a desired goal-directed behaviour in a specific situation and can be used as a strategy to break habitual behaviour (Jager et al., 1996). Using prompting as a technique to stimulate stair use builds on the assumption that the alternative behaviour, elevator/ escalator, has a strong habitual component. Habits are formed when a specific goal-directed behaviour is selected and performed frequently, leading to associations between the goal and behaviour (Aarts \& Dijksterhuis, 2000a). The association allows an automatic behavioural response upon activation of these goals by the environment (Aarts \& Dijksterhuis, 2000a; Aarts \& Dijksterhuis, 2000b). In order to prevent or reduce undesirable habitual behaviours, such as elevator use, the automatic link between goal and behaviour needs to be broken (Sheeran et al., 2005a). As habits are automatic behavioural routines elicited in the same environment, changing the environment by prompting may obstruct the automatic link and therefore 'break' the habit (Sheeran et al., 2005a).

In spite of the success of prompts in increasing stair use in commercial settings, the effectiveness of prompts in worksite settings is less clear (Eves \& Webb, 2006a). Worksite settings in which stair promotion interventions have been conducted are either office buildings (Coleman \& Gonzalez, 2001; Kerr et al., 2001e; Kerr et al., 2004; Titze et al., 2001; Vanden Auwelee et al., 2005) or health care related facilities (Marshall et al., 2002). To date no studies have examined the effectiveness of prompts to promote stair use in blue-collar worksites. Blue-collar workers are a neglected group in worksite interventions (Bagwell \& Bush, 2000). Increasing stair use among blue-collar workers seems important, as they are at higher risk for cardiovascular disease (Bennet, 1996) and are more likely to be obese (Sarlio-Latheenkorva \& Lahelma 1999; Galobardes et al., 2000). Moreover, occupational physical activity has declined substantially over the past decade (Brownson et al., 2005), including that of the traditionally more-active occupational groups (Moller et al., 1991). As blue-collar workers report lower levels of participation in leisure-time physical activity than white-collar workers (Crespo et al., 1999; Burton \& Turrell, 2000), it seems less likely that they will compensate for the decrease of occupational physical activity.

There is a lack of knowledge regarding the exact decision-making processes involved in taking the stairs or elevator of blue-collar workers. Previous studies that have investigated the effectiveness of prompts in white-collar worksites found time, accessibility, attractiveness and number of stairs to be important considerations in the choice of stairs or elevator (Kerr et al., 2001e; Titze et al., 2001). However, other factors may influence this choice among blue-collar workers, which might not be susceptible to the influence of prompting. The present study will therefor test whether an intervention using prompts to increase stair use, is effective in stimulating stair use in two types of worksites: one consisting mainly of bluecollar workers and one mainly of white-collar workers. 


\section{METHODS}

\section{Settings and participants}

One office building (approximately 150 white-collar employees; 36.9\% women) and one paper factory (approximately 800 mainly blue-collar employees; $2.5 \%$ women), located in the Netherlands, were chosen as intervention sites. Both worksites are part of a larger scale study directed at weight gain prevention in healthy young adults at the worksite (NHF-NRG In Balance-project, for more information see Kwak et al., 2007b). The present intervention was implemented 14 months after the start of the overall intervention. Prior to implementation participants of both worksites had received individual interventions (e.g. feedback on body composition, pedometer and computer tailoring) and were exposed to environmental interventions (e.g. lunch-walking and active transport schemes). No interventions were implemented concurrent with the present intervention.

The office building was typical of most office buildings, with mostly sedentary occupations and equal types of jobs for men and women. The paper factory, which manufactures paper from pulp, was partly automated; typical job duties include packing, lifting and working machines. Job duties of machine operators varied dependent on the product quality; during machine malfunction this would include frequent use of the machinery stairs (1.5-2.0 meters). Women mainly performed office work. Occupational physical activity did not differ within workers; every worker had fixed job duties, which were constant over time. Employees of both worksites had equal choice to take the stairs or elevator; the only job factor limiting this choice was transporting heavy/large load.

The office building (9 floors) contained one stairwell and two elevators, located in the hallway at the building entrance within sight of each other. The stairway was white, had 9 steps (18cm high, $27 \mathrm{~cm}$ deep and $127 \mathrm{~cm}$ in width), a landing and 9 more steps, all covered with dark grey carpet. The factory ( 5 floors) had one stairwell and one elevator, which were adjacent and situated at the building entrance. The stairwell, with tiled walls (light brown), separated manufacturing space from office space; with the canteen on the fifth floor. The stairway had 13 steps (16cm high, $27 \mathrm{~cm}$ deep and $147 \mathrm{~cm}$ in width), a landing and 13 more steps, all uncovered dark stone. The stairways of both buildings were spacious; every landing had a window and a big light. The atmosphere differed; the stairway in the factory was bare and old, noises and odours penetrated from the manufacturing space, these were not noticeable in the elevator.

\section{Design}

During a three-week intervention period, an A5-sized poster was placed at the entrance of the elevator of every floor and an A2-sized poster was placed in the stairwell on every floor. All posters had the same design; a green-coloured background with black text. There were four different versions of the A5-sized poster containing one of the following four slogans: "Free workout?", "Walking buffet?, 'A break?" and "Dessert?". The A2-sized poster had two different versions: "One step closer to your energy balance" or "On your way to the balance between food intake and physical activity". All posters ended with "The stairs. A good idea!". The study used a simple time-series design of collecting data in three waves: before, during and after implementation of the posters. Elevator and stair use was monitored by an observer, who was inconspicuously stationed in the ground floor hallway of each worksite, within direct view of the elevator and stairs. For the baseline monitoring, the observer recorded the number of men and women using the elevator and stairs in the morning between 8.00 and 10.00 and at lunchtime between 12.00 and 14.00 three days per 
week over a period of 2 weeks. Subjects carrying heavy/large luggage (larger than a briefcase/medium-sized bag) were excluded, because of the potential influence on the decision process. During the whole intervention period observations were carried out with an identical procedure. Data collection for the follow-up measurements started one week after the prompts had been removed and followed the same protocol for two weeks. During data-collection the stairs were used to go to one's office/workspace (including shift change), lunch breaks and normal job duties, such as meetings and checking machines. Of the 800 workers at the factory, 120 individuals do not work shifts; the rest is divided over 5 shifts. Only the individuals not working shifts and those of the morning and day shifts could be observed, observations of shifts varied from 3-21 days.

After the intervention period was stopped a random-selected group of employees of the worksites received a process-evaluation questionnaire. Respondents were asked if they had seen the poster and if so to indicate the colour and text of the poster (multiple choice) and to express their attitude towards the poster (fivepoint scale: 'very positive'-'very negative'). They also indicated if their choice to take either elevator or stairs had been influenced by the posters (binary scale) and if they usually took the elevator or stairs. Participants were also asked what their main reason was for their choice (open-ended).

\section{Statistical analysis}

Data were analyzed using SPSS 11.5 statistical package. Logistic regression analyses were performed with stair use as dichotomous dependent variable and gender, worksite and intervention period (baseline, intervention, follow-up) as independent. Gender was considered an important confounder, as research has demonstrated significant gender effects (Blamey et al., 1996; Russell et al., 1999). Direction of travel, load and traffic volume were not selected as confounders as the study focused on overall stair use, independent of direction of travel, on individuals with an equal choice to use the stairs, not influenced by load and because no variations in traffic volume were expected during the observation period. Interactions between the intervention period and gender, and the intervention period and worksite were explored. In order to test the different periods, baseline was compared with intervention, intervention with follow-up and baseline with follow-up. The self-report process data were analyzed descriptively.

\section{RESULTS}

In total 6771 choices between stairs and elevator were observed $(16.1 \%$ women and $83.9 \%$ men). There was a significant difference between stair use at baseline (32.6\%) and during the poster intervention (37.7\%; see figure 1$)$. Overall, women used the stairs more than men and white-collar workers used the stairs more than blue-collar workers. There was no significant gender-by-intervention interaction. The absence of a worksite-by-intervention interaction implied that the prompting intervention effect did not differ between the white- and blue-collar worksites $(2.6 \%$ and $4.4 \%$ increase respectively; see table 1 and table 2). 
Figure 1. Stair use during baseline, intervention and follow-up, ${ }^{* * *} \mathrm{p}<.001,{ }^{* *} \mathrm{p}<.01$.

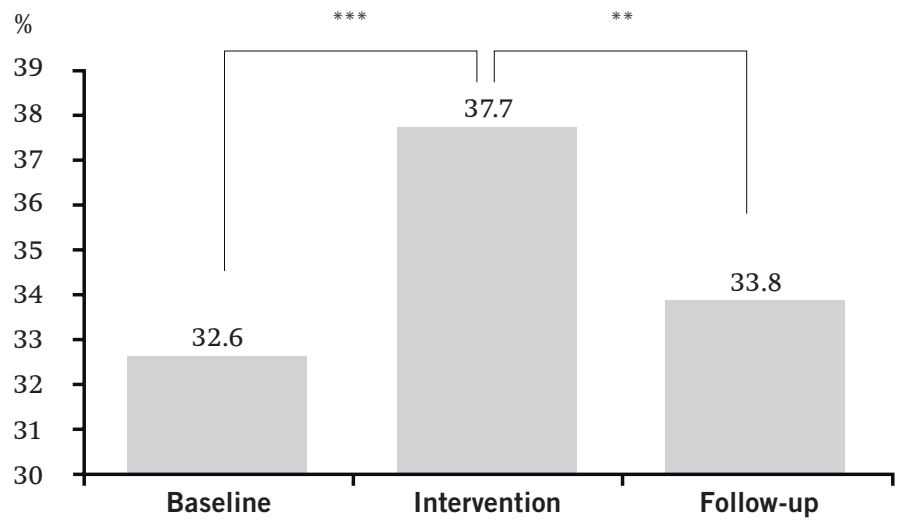

Table 1. Percentages for stair use compared with baseline separated for gender and type of worksite.

\begin{tabular}{llll} 
Intervention site & Baseline (\%) & Intervention (\%) & Follow-up (\%) \\
Overall & $32.7(\mathrm{n}=684)$ & $37.7(\mathrm{n}=1012)$ & $33.8(\mathrm{n}=673)$ \\
\hline Men & $29.9(\mathrm{n}=521)$ & $35.7(\mathrm{n}=802)$ & $32.4(\mathrm{n}=548)$ \\
\hline Women & $46.6(\mathrm{n}=163)$ & $47.7(\mathrm{n}=210)$ & $41.5(\mathrm{n}=125)$ \\
\hline Blue-collar worksites & $24.0(\mathrm{n}=302)$ & $28.4(\mathrm{n}=405)$ & $24.5(\mathrm{n}=304)$ \\
\hline White-collar worksites & $45.6(\mathrm{n}=381)$ & $48.2(\mathrm{n}=607)$ & $49.2(\mathrm{n}=369)$ \\
\hline
\end{tabular}

Note. $n=$ number of observations.

During follow-up overall stair use decreased significantly relative to the intervention phase (33.8\%). Again, there was an effect of gender, but no significant gender-by-intervention interaction. The difference in stair use between baseline and follow-up was not significant. During follow-up women and white-collar workers used the stairs more than respectively men and blue-collar workers. There was no significant gender-by-intervention interaction. Even though there was no worksite-by-intervention interaction, stair use remained elevated at follow-up in the white-collar worksite, while it returned near baseline at the blue-collar site.

Table 2. Odds Ratios for main comparisons.

\begin{tabular}{llll} 
Main effects & $\begin{array}{l}\text { Intervention } \\
\text { vs baseline }\end{array}$ & $\begin{array}{l}\text { Follow-up vs } \\
\text { intervention }\end{array}$ & $\begin{array}{l}\text { Follow-up vs } \\
\text { baseline }\end{array}$ \\
\hline Effects of prompting & $1.19(1.05,1.34)$ & $0.64(0.43,0.94)$ & $1.04(0.98,1.12)$ \\
\hline Women vs men & $1.38(1.17,1.62)$ & $1.23(1.04,1.45)$ & $1.30(1.09,1.56)$ \\
\hline $\begin{array}{l}\text { White-vs blue-collar } \\
\text { worksite }\end{array}$ & $2.32(2.05,2.63)$ & $2.51(2.21,2.84)$ & $2.67(2.33,3.07)$ \\
\hline
\end{tabular}

Self-report data were obtained from a self-selected sample of 129 employees (34 blue-collar (22.7\%) and 85 white-collar workers (70.8\%); see table 3). Significantly more blue-collar workers $(88 \%)$ reported seeing the poster than white-collar workers 
(67\%). The attitude towards the poster did not differ significantly between blue- and white-collar workers; on average a neutral to positive attitude was reported. The percentage of blue-collar workers that correctly recognized the colour of the poster was significantly higher than the percentage of white-collar workers (50\% and 20\% respectively); this was also the case for correctly recognizing the text. Of the respondents that had seen the poster $29 \%$ of blue-collar and $19 \%$ of white-collar workers reported that their choice to take the stairs had been influenced by the poster. Half of the white-collar workers usually took the stairs; significantly higher than that reported by the blue-collar workers. Time and number of floors were reported as important considerations in the choice to take either the stairs or elevator. Additionally, easiness was given as a reason for taking the elevator and being physically active as a reason for taking the stairs. The reported reasons did not differ significantly per type of worksite.

Table 3. Self-reported process measures per type of worksite.

\begin{tabular}{|c|c|c|c|}
\hline & $\begin{array}{l}\text { Blue-collar } \\
\text { worksite } \\
(n=34)\end{array}$ & $\begin{array}{l}\text { White-collar } \\
\text { worksite } \\
(n=85)\end{array}$ & \\
\hline & $\%$ & $\%$ & $\mathrm{X}^{2}$ \\
\hline Seen poster & 88 & 67 & $5.54^{*}$ \\
\hline \multicolumn{4}{|l|}{ Attitude towards poster } \\
\hline mean, (SD) & $2.32(.65)$ & $2.59(.72)$ & $\mathrm{t}=1.75$ \\
\hline Recalled correct colour & 50 & 20 & $8.57^{* *}$ \\
\hline Recalled correct text & 77 & 55 & $4.29^{*}$ \\
\hline Choice influenced by poster & 29 & 19 & 1.27 \\
\hline Usually takes stairs & 22 & 50 & $7.28^{* * *}$ \\
\hline \multicolumn{4}{|l|}{ Main reasons for taking the lift } \\
\hline Number of floors & 42 & 32 & 0.53 \\
\hline Easiness & 17 & 24 & 0.40 \\
\hline Time & 17 & 15 & 0.04 \\
\hline \multicolumn{4}{|l|}{ Main reasons for taking the stairs } \\
\hline Being physically active & 17 & 37 & 0.98 \\
\hline Number of floors & 17 & 26 & 0.23 \\
\hline Time & 17 & 16 & 0.00 \\
\hline
\end{tabular}

Note. ${ }^{*}$ Differ significantly at $p<0.05,{ }^{* *} p<0.01,{ }^{* * *} p<0.001$ between blue-collar and white collar worksites.

\section{DISCUSSION}

The present study investigated whether an intervention using prompts on posters was effective in stimulating stair use in a white-collar worksite and in a bluecollar worksite. The results show that overall prompts had a short-term effect in increasing stair use of approximately 5\%. Previous studies that have tested the effects of prompts on stair usage in worksite settings found similar short-term effects varying from 1\% (Marshall et al., 2002) to 8\% (Vanden Auwelee et al., 2005). This is however the first study that has not only investigated the effects of prompts on stair use in a white-collar worksite, but also in a blue-collar worksite. No significant worksite-by-intervention interaction was found, the prompts were equally effective in both types of worksites. Self-report data, collected from a small 
proportion of the employees, indicated that blue-collar workers were more likely to have seen the poster and to have correctly recalled the colour and text of the poster. Ensuring the availability and accessibility of stairwells in blue-collar worksites can thus provide a low-cost and convenient way to integrate physical activity into the lifestyles of blue-collar workers.

The theoretical reason behind the effectiveness of prompts is that they disrupt habitual behaviour by altering the context within which that behaviour normally occurs, thereby prompting individuals to re-evaluate their habitual response (Kerr et al., 2001a). Jager and colleagues (1996) suggest that a prompt will only be effective when it can bring existing strong beliefs into consciousness, even without requiring substantial levels of attention or intention. A prompt directed at a belief that does not exist, cannot be made salient, thus precluding any behavioural effect in that individual (Jager et al., 1996). Additionally, it has been found that prompts containing general description messages, in contrast to providing reasons to act, will have no effect beyond that already induced by a response-specific prompt (Smith \& Bennet 2002). Using prompts that do not provide reasons for taking the stairs, like those used in the present study, could be more likely to coincide with existing beliefs of a broader population and have a more homogenous effect on the public's response, than prompts related to very specific benefits, such as 'Watch your step, Use the stairs, Do it! For the life of your family!' (Coleman \& Gonzalez 2001). Observational studies are needed in which the effects of message contents are analyzed (Webb \& Eves 2007).

Additionally further studies should identify potential moderators of the effect, such as the importance of self-efficacy (Jager et al., 1996). The effect of prompts might be limited to behaviours that are easy to perform, such as taking the stairs to the first floor. Prompts might be less effective in stimulating more challenging behaviours such as taking the stairs to the fifth floor. Self-report data of previous studies indicated that employees on lower floors used the stairs more than co-workers on higher floors (Kerr et al., 2001e). The results of the self-report data of our study support this notion, as the number of floors was given as the main reason for taking the elevator. Further research should also investigate the possibilities of prolonging the effects, an increase in stair use was observed during follow-up, however not significant.

The present study had various limitations. The first limitation pertains to the research population; both worksites were part of a larger study directed at the prevention of weight gain (Kwak et al., 2006a). The study therefore is confined to a specific sample of persons, who most likely possess the intention to change their dietary and physical activity behaviour (Kwak et al., 2006a). The observed results can therefore not simply be generalized to all kind of settings or persons. Apart from the intentions related to energy balance-related behaviours, participants seem a representative sample of the population, regarding variables such as gender and age. Secondly, the larger study also contained other health promotion interventions, which limits the generalizability of the stair intervention alone. However, the present study does show that prompting had an additional effect above that induced by the overall intervention. This corresponds with recent findings of Eves and Webb (2006a), who reported in a recent review that most of the studies provided some encouragement for increasing stair use in worksite settings by using prompts, but particularly with additional inducements such as improved aesthetics and music (Eves \& Webb 2006a). An additional limitation to generalizability is that factories differ in physical configuration and required amounts of occupational physical activity. Thus, this factory under more or less hectic production conditions might have different potential rates of stair use and 
other factories may be altogether different from this site. A third limitation is related to the generalizability of the survey data, the survey sample was selfselected and had a very small response rate among factory workers. These data should therefore be interpreted with caution. A final limitation is that during data collection no distinction was made between stair ascent and descent. Eves and Webb (2006a) emphasize the importance of distinguishing these two and to mainly stimulate stair climbing, as stair climbing uses three times the energy expenditure of stair descent (Eves \& Webb 2006a).

\section{CONCLUSION}

In conclusion, the present study shows that individuals at both blue- and whitecollar worksites can be effectively stimulated to take the stairs with a short low cost intervention using prompts on posters. Additionally, the results of the present study provide support for the importance of environmental cues as a central part in health-promoting activity. 



\section{CHAPTER 7}

THE EFFECTIVENESS OF THE NHF-NRG IN BALANCE-PROJECT: SHORT-AND LONG-TERM CHANGES IN SUM OF SKINFOLDS AND WAIST CIRCUMFERENCE

Submitted as: Kwak, L., Kremers S.P.J., Candel, M.J.J.M, Visscher, T.L.S., Brug, J. \& Van Baak, M.A. The effectiveness of the NHF-NRG In Balance-project: short-and long-term changes in sum of skinfolds and waist circumference 


\section{ABSTRACT}

Despite expanding interest in weight gain prevention, little is known about the effectiveness of weight gain prevention programmes, as few studies have tested programmes that were designed for this purpose. The aim was to test the short-and longer-term effectiveness of the NHF-NRG In Balance-project, a worksite-based intervention aimed at the prevention of weight gain, with regard to changes in sum of skinfolds, waist circumference, body weight and BMI. A quasi-experimental pretest-multiple post-test control group design was applied in 12 worksites $(n=570)$. The intervention group ( 6 worksites) received individual and environmental interventions, directed at both physical activity and food intake. Implementation started in September 2003-September 2004. Anthropometric measures (sum of skinfolds, waist circumference, weight and height) were measured at baseline, and 1 and 2 years after baseline. Linear regression analysis showed a significant greater reduction in sum of skinfolds after 12 months in the intervention group $(-7.4 \pm 11.5 \mathrm{~mm})$ than in the control group $(-4.7 \pm 9.1 \mathrm{~mm}, \mathrm{p}<0.05)$. These differences sustained over 24 months $(\mathrm{p}<.001)$. A larger reduction was observed in women both at 12 months, as 24 months. Significant differences were also observed between the intervention and control group for changes in waist circumferences both at 12 months $(-1.2 \pm 4.9 \mathrm{~cm}$ and $0.3 \pm 3.3 \mathrm{~cm}, \mathrm{p}<.001)$ as 24 months $(\mathrm{p}<.01)$. In conclusion, the findings presented show the effectiveness of the NHF-NRG In Balance-project and supports the usefulness of prevention, especially regarding long-term maintenance. 


\section{INTRODUCTION}

Data from around the world show alarming increases in the prevalence of obesity during past decades (WHO, 2000). The situation in the Netherlands is no exception, with comparable increases in the number of individuals suffering from overweight and obesity as other European countries. At present, approximately $40 \%$ of Dutch adults are overweight, of which $10 \%$ is obese. If this trend continues, it is estimated that 15 to $20 \%$ of adults in the Netherlands will be obese by 2015 (Kemper et al., 2004). In order to tackle this health issue increasing emphasis is being placed on primary prevention of obesity (Klem et al., 2000; Eiben \& Lissner, 2006), as the traditional weight loss programmes are often unsuccessful on the long-term (Miller, 1999). Despite the expanding interest, little is known about the effectiveness of weight gain prevention programmes, as few studies have tested programmes that were designed for this purpose (Hardeman et al., 2000; Klem et al., 2000).

The multidisciplinary research programme 'Netherlands Research programme weight Gain prevention' (NHF-NRG) was initiated for exactly this purpose and aimed to evaluate three weight gain prevention programmes targeted at different risk-groups (Kremers et al., 2005a). The present paper describes the effectiveness of one of these programmes, namely the NHF-NRG In Balance-project: a worksite-based prevention programme directed at the prevention of weight gain in young adults, through changes in both physical activity and food intake (Kwak et al., 2007b). Young adults are of particular relevance for prevention of weight gain, as young adulthood is recognized as a high-risk period for weight gain (Sherwood et al., 2000; Sheehan et al., 2003). The average annual weight gain of young adults in the Netherlands is approximately $0.60 \mathrm{~kg} / \mathrm{year}$ (Visscher et al., 2002). The study was worksite-based, not only as this is a major environmental context for young adults (Dishman et al., 1998), but also because worksites provide many opportunities to reinforce the adoption and maintenance of healthy lifestyle behaviours (Kremers et al., 2005a).

The present paper reports the short-and longer-term follow-up results of the NHF-NRG In Balance-project on several anthropometric measures, which include body weight, body mass index (BMI), sum of skinfolds and waist circumference.

\section{METHODS}

\section{Participants}

The recruitment of participants included two steps. First, worksites were recruited based on data collected through the Chamber of Commerce. Eligible worksites should have a minimum of 100 employees and provide canteen facilities. In total, 128 worksites were approached by mail and telephone and invited to participate, twelve of which were recruited (Kwak et al., 2006a). The twelve recruited worksites were located in the south of the Netherlands; two sites were hospitals, two were local governments, five were factories (paper-factory, tile-factory, pigmentfactory, furnish manufacture and cable-factory), one university, one water-supplying company and one energy-supplying company. Each of these worksites employed between 100-800 employees. The second part of the recruitment concerned the individual participants. With permission of the participating worksites, employeeswerecontacted byletter and invited toparticipatein a weightgain prevention programme. To be eligible for the study participants should have a BMI above 18 $\mathrm{kg} / \mathrm{m}^{2}$. Exclusion criteria were medical restrictions with regard to diet or physical activity behaviour. Interested individuals received an information booklet in 
which the project was described in more detail and which included an informed consent form. All participants read and signed the informed consent form before participating in the study. The study was approved by the Medical Ethical Committee of the Academic Hospital Maastricht, the Netherlands.

\section{Study design}

The study had a non-randomized pre-test repeated post-test control group design. Worksites were matched with respect to social economic status of the employees and assigned to either the intervention or control group (see also Kwak et al., 2006a). Worksites and participants in the intervention group received the NHF-NRG In Balance-intervention for a period of 1-year. Individuals in the control group were only contacted for baseline and follow-up measurements. Outcome measures were assessed at baseline, 12 and 24 months and included body composition measures and self-report measures. The protocol for the measurements was identical for all three time-points and for all subjects. All measurements were executed in the morning after an overnight fast, at the worksite of the participant and performed by the same researcher. The self-report measures were assessed with a self-administered written questionnaire, which participants returned completed during the body composition measurements. Measurements started in September 2003 and data collection was completed for all study participants in August 2006.

\section{The NHF-NRG In Balance-project intervention}

A complete description of the intervention of the NHF-NRG In Balance-project has been published previously (Kwak et al., 2007b). Briefly, the programme, which was developed based on the Intervention Mapping protocol (Bartholomew et al., 2001), aimed to prevent weight gain through the following programme objectives: (1) increase frequency and duration of walking and cycling, (2) increase physical activity level at work, (3) decrease portion sizes and (4) decrease intake of energy-dense foods, through replacement of high fat products by low energy dense products, replacement of products low in fibre by fibre-rich products and replacement of saturated fats by unsaturated fats. The programme consisted of an individual component and a worksite (environmental) component. The components were both directed at changes in food intake and physical activity. The individual component contained several interventions, including feedback on measured body composition measures, self-monitoring devices such a pedometer and diaries, a website and computer tailoring. The environmental components were incorporated into a handbook, which served as a guide for assisting a worksite linkage board through the different stages of diffusion of the environmental interventions. Interventions included for example changes in the assortment of food products in the cafeteria, workshops, posters/ prompts stimulating stair use and ways to form lunch-walking and cycling groups. Environmental interventions were modified to fit within specific worksites.

\section{Outcome measures}

Body weight, height, sum of skinfolds and waist circumference were measured. Body weight $(\mathrm{kg})$ was measured, in underwear, to the nearest $0.1 \mathrm{~kg}$ with a digital laboratory scale (Seca, Model 861, Hamburg, Germany). Height (m) was measured to the nearest $1.0 \mathrm{~mm}$ without shoes with a mobile measuring unit (Seca, Model 225, Hamburg, Germany). The sum of skinfolds was determined using the sum of four skinfolds measured with the Harpenden skinfold calliper (HSK-BI, British Indicators, West Sussex, UK). Skinfolds included biceps (anterior surface of the biceps midway between the anterior auxiliary fold and the antecubital fossa), triceps (vertical fold on the posterior midline of the upper arm, halfway between the acromion 
and olecranon process), subscapular (fold on the diagonal line coming from the vertebral border to between 1 and $2 \mathrm{~cm}$ from the inferior angle of the scapulae) and subrailiac (diagonal fold above the iliac crest even with the anterior auxiliary line). Waist circumference measures were obtained to the nearest $0.1 \mathrm{~cm}$ with a flexible tape measure. Waist circumference was measured at the abdominal waist (horizontal at the umbilicus).

The following self-report outcome measures obtained by the questionnaire were incorporated in the present study: demographic characteristics including gender, date of birth, marital status (married or living together; separated, divorced, widowed, never married) and highest level of education (high school or less; some college/vocational training; university); smoking status, measured with the following item: “do you smoke?” (yes, daily; yes, occasionally; no, never).

\section{Statistical analyses}

A required sample size of 500 participants from 12 worksites was determined to be large enough to detect a weight difference between the groups of $0.6 \mathrm{~kg}$, with a power of $80 \%$ and an alpha of $5 \%$. The difference of $0.6 \mathrm{~kg}$ is based on the expected weight gain in this age group in the Netherlands in one year (Visscher et al., 2002). The primary outcomes examined in this study are changes in body weight, BMI, sum of skinfolds and waist circumference from baseline to 12 months and 24 months. Prior to the analyses, data of female participants who had been pregnant during the two-year project were excluded. To assess potential dropout bias, baseline characteristics age, gender, BMI, marital status, education and smoking status were compared between those who dropped-out and those who attended all measurements. An intention-to-treat analysis was conducted for 12 and 24 months in which dropouts in the intervention and control group were assigned average weight changes that were observed in the control group at both time-points.

The effect analyses were performed in four steps. First, differences in baseline characteristics of participants in the intervention and control group were explored, using Student's t-test or Chi-square test. Second, differences between the intervention and control group in changes in body weight, BMI, sum of skinfolds and waist circumference at 12 and 24 months were examined using linear regression analyses adjusting for various baseline characteristics (age, gender, BMI, marital status, education and smoking status). Third, Cohen's $d$ effect sizes were calculated in order to calculate the magnitude of the intervention effect; $d$ is defined as the difference between two means divided by the pooled standard deviation for those means (Cohen, 1988). Fourth, potential interaction effects of the intervention group with gender and BMI were explored. If significant interactions occurred analyses were repeated with stratification by gender, age, or BMI.

As a consequence of the study-design participants were nested within worksites. It was expected that a high probability of interdependence would occur between participants of the same worksite. To take this into account, all multilevel linear regression analyses were conducted in MlwiN with random intercepts (Rabash et al., 1999), this in order to assess the effects of the intervention on changes in body weight, BMI, sum of skinfolds and waist circumference. All p-values are two-sided and 5\% level of significance was used.

\section{RESULTS}

\section{Participant characteristics}

Baseline characteristics of the control and intervention group are described in 
table 1. Participants from the intervention group were older and had a higher BMI than participants from the control group (38.9 vs. 35 years and $25.7 \mathrm{vs.} 24.2 \mathrm{~kg} / \mathrm{m}^{2}$ ). Apart from these differences groups did not differ in terms of baseline characteristics. Overall, there was an equal distribution of men and women between the intervention and control group and participants were equally well educated, married or living together and non-smokers.

Table 1. Baseline characteristics of participants.

\begin{tabular}{|c|c|c|c|}
\hline & $\begin{array}{l}\text { Control } \\
(n=188)\end{array}$ & $\begin{array}{l}\text { Intervention } \\
(n=365)\end{array}$ & P-value \\
\hline Age, years mean (s.d.) & $35.0(7.4)$ & $38.9(8.2)$ & $<.01$ \\
\hline Gender (\% female) & 48.2 & 50.7 & .61 \\
\hline \multicolumn{4}{|l|}{ Highest education (\%) } \\
\hline High school or less & 19.3 & 15.7 & .41 \\
\hline Some college/vocational training & 29.2 & 34.7 & \\
\hline University & 51.6 & 49.6 & \\
\hline \multicolumn{4}{|l|}{ Marital status (\%) } \\
\hline Married or living together & 77.1 & 83.9 & .06 \\
\hline \multicolumn{4}{|l|}{ Separated, divorced, } \\
\hline widowed or never married & 22.9 & 16.1 & \\
\hline Current smoker (\%) & 17.3 & 16.0 & .72 \\
\hline Body Mass Index, $\mathrm{kg} / \mathrm{m}^{2}$ mean (s.d.) & $24.2(3.1)$ & $25.7(4.0)$ & $<.01$ \\
\hline
\end{tabular}

Note: Abbreviation: s.d., standard deviation. P-value estimated by t-test, with exception for gender, which was estimated with Chi-square test.

\section{Attendance}

The number of participants who were not measured at 12 and 24 months was 68 (18.7\%) and 107 (29.5\%), respectively, for the intervention group and $23(12.2 \%)$ and $42(22.3 \%)$, respectively, for the control group. The most common reasons for discontinuation were change of occupation, conflict with workload and mental problems. The dropout analyses revealed some selective dropout. Both after 12 and 24 months smokers were more likely to discontinue the study than non-smokers (OR=1.87, 95\% C.I. 1.02-3.43 and $\mathrm{OR}=2.33$, 95\% C.I. 1.41-3.84). The intention-to-treat analyses (data not presented) showed similar results as those presented below both for the changes after 12 and 24 months.

\section{Anthropometric changes over one and two years}

Changes in sum of skinfolds, waist circumference, body weight and BMI for the two groups over 12 and 24 months are depicted in table 2. At 12 months followup, a statistically significant difference between the intervention and control group was evident for the change of sum of skinfolds ( $B=-2.52$, 95\% C.I. $-4.58,-0.45)$, with a corresponding Cohen's $d$ of 0.26 . A greater reduction in sum of skinfolds was observed for participants in the intervention group than for participants in the control group. These differences sustained over 24 months ( $\mathrm{B}=-4.83,95 \%$ C.I. $-6.98,-2.67$ ), the Cohen's $d$ was 0.44 . Significant differences were also observed between the intervention and control group for changes in waist circumferences both at 12 months $(\mathrm{B}=-1.50$, 95\% C.I. $-2.35,-0.65)$, as 24 months $(\mathrm{B}=-1.30$, 95\% C.I. $-2.18,-0.42)$, with respectively the following Cohen's $d 0.37$ and 0.33 . Participants from the intervention group reduced their waist circumferences over time in comparison to an increase in 
the control group. Changes in weight and BMI did not differ significantly between the two groups neither over 12 or 24 months. Although changes were not statistically significant, all observations were in favour of the intervention group. The corresponding Cohen's d's were all smaller than 0.20.

Table 2. Changes in anthropometric variables over time in the intervention and control group.

\begin{tabular}{|c|c|c|c|c|c|c|c|}
\hline \multirow[t]{2}{*}{ Variable } & \multicolumn{2}{|c|}{ Intervention } & \multicolumn{2}{|c|}{ Control } & \multirow[t]{2}{*}{ B } & \multirow[t]{2}{*}{ 95\% C.I. } & \multirow[t]{2}{*}{ Cohen's $d$} \\
\hline & No. & Mean (s.d.) & No. & Mean (s.d.) & & & \\
\hline \multicolumn{8}{|c|}{$\begin{array}{l}\text { Sum skinfolds } \\
(\mathrm{mm})\end{array}$} \\
\hline 12 months & 294 & $-7.4(11.5)$ & 164 & $-4.7(9.1)$ & $-2.52^{*}$ & $-4.58,-0.45$ & 0.26 \\
\hline 24 months & 255 & $-10.0(12.6)$ & 145 & $-5.0(10.4)$ & $-4.83^{* * *}$ & $-6.98,-2.67$ & 0.44 \\
\hline \multicolumn{8}{|l|}{ Waist, cm } \\
\hline 12 months & 295 & $-1.2(4.9)$ & 164 & $0.3(3.3)$ & $-1.50^{* * *}$ & $-2.35,-0.65$ & 0.37 \\
\hline 24 months & 256 & $-0.5(5.6)$ & 145 & $1.0(3.4)$ & $-1.30^{* *}$ & $-2.18,-0.42$ & 0.33 \\
\hline \multicolumn{8}{|l|}{ Weight, kg } \\
\hline 12 months & 295 & $-0.64(3.32)$ & 165 & $-0.13(2.29)$ & -0.43 & $-1.08,0.22$ & 0.18 \\
\hline 24 months & 256 & $-0.29(4.25)$ & 146 & $0.08(3.16)$ & -0.17 & $-0.84,0.51$ & 0.10 \\
\hline \multicolumn{8}{|l|}{ BMI, $\mathrm{kg} / \mathrm{m}^{2}$} \\
\hline 12 months & 294 & $-0.23(1.2)$ & 164 & $-0.04(0.8)$ & -0.14 & $-0.35,0.08$ & 0.19 \\
\hline 24 months & 255 & $-0.11(1.4)$ & 145 & $0.03(1.0)$ & -0.05 & $-0.05,0.23$ & 0.12 \\
\hline
\end{tabular}

Note: Random intercept at worksite level, adjusted for baseline age, gender, BMI, education, marital status and smoking status. P-values for differences between intervention and control groups, ${ }^{*} p<0.05,{ }^{* *} p<.01$ and ${ }^{* * *} p<.001$.

Significant interaction terms were found for the changes in sum of skinfolds (table 3). A significant interaction effect of group with gender was observed for the changes in sum of skinfolds at $12(\mathrm{p}<.05)$ and 24 months $(\mathrm{p}<.05)$, on the basis of which a stratification was made by gender. A larger reduction in sum of skinfolds was observed in women in the intervention group than women in the control group both at 12 months $(\mathrm{B}=-3.15$, 95\% C.I. -5.95 , -0.35$)$, as 24 months $(\mathrm{B}=-5.14,95 \%$ C.I. -8.06 , -2.21). No significant effects were observed among men.

Table 3. Body composition changes stratified for gender adjusted for baseline age, BMI, marital status, education and smoking status.

\begin{tabular}{lllll} 
I Men & \multicolumn{4}{l}{ Women } \\
& C & B & C & B \\
& & & & $95 \%$ C.I.
\end{tabular}

\begin{tabular}{lllllll}
$\begin{array}{l}\text { Sum of } \\
\text { skinfolds }(\mathrm{mm})\end{array}$ & \multicolumn{7}{l}{} & & & \\
\hline 12 months & $-6.0 \pm 10.8$ & $-5.4 \pm 7.5$ & 1.27 & $-8.6 \pm 12.0$ & $-3.8 \pm 10.5$ & $-3.15^{*}$ \\
& $(\mathrm{n}=144)$ & $(\mathrm{n}=86)$ & $-1.60,4.13$ & $(\mathrm{n}=150)$ & $(\mathrm{n}=78)$ & $-5.95,-0.35$ \\
\hline 24 months & $-7.4 \pm 12.0$ & $-5.3 \pm 10.1$ & 0.06 & $-12.6 \pm 12.8$ & $-4.7 \pm 10.7$ & $-5.14^{* *}$ \\
& $(\mathrm{n}=128)$ & $(\mathrm{n}=76)$ & $-2.96,3.08$ & $(\mathrm{n}=127)$ & $(\mathrm{n}=69)$ & $-8.06,-2.21$ \\
\hline
\end{tabular}

Note: Abbreviations: I, intervention group; $C$, control group. ${ }^{*} p<.05,{ }^{* *} p<.001$ for difference between the intervention and control group. 


\section{DISCUSSION}

The present study was designed to test the short-and longer-term effectiveness of the NHF-NRG In Balance-project, with regard to changes in sum of skinfolds, waist circumference, body weight and BMI. The results indicate that with regard to changes in sum of skinfolds and waist circumference the project was indeed effective both at 12 and 24 months. Even though changes in weight and BMI between the intervention and control group were not significantly different, they did change in the desired direction. Overall, the intervention of the NHF-NRG In Balance-project had a positive effect on the body composition measures of the individuals in the intervention group. The interpretation of effect sizes of Cohen's $d$ imply effects of medium magnitude for the changes in sum of skinfolds and waist circumference both after 12 and 24 months (Cohen's $d$ between 0.33 and 0.55). Such changes in body composition indicators may have important health implications, as it has been demonstrated that the health risks associated with obesity derive primarily from fat rather than weight (Wells \& Victoria, 2005). Moreover, it is not only the total amount of fat that is important, but also the distribution of fat in the body (Pi-Sunyer, 2000), with central fatness being most related to health risks (Seidell et al., 1987). The reduction in sum of skinfolds and waist circumference observed in the present study reflects a reduction in central fatness (Lemieux et al., 1996; Wells \& Victoria, 2005). The decrease in waist circumference is most relevant, as a large waist circumference is independently associated with health risks (Chan et al., 1994; Rimm et al., 1995) and mortality (Baik et al., 2000; Visscher et al., 2001). Recent studies have shown that waist circumference is increasing even more rapidly than BMI (Chen \& Tunstall-Pedoe, 2005).

The observed changes in anthropometric measures could be a result of changes in participants' food intake and/or physical activity behaviour. With regard to changes in waist circumference it has been demonstrated that an increase in fibre intake was associated with a reduction in waist circumference in men (Koh-Banerjee et al., 2003). A strong dose-response relationship was demonstrated between the amount of exercise and measures of central obesity (Slentz et al., 2004). Interestingly, changes in physical activity can lead to changes in body composition, which may be reflected in changes in waist circumference, while body weight remains stable through increased muscle mass (Wilmore et al., 1999; Ross et al., 2000). This is in line with the findings of the present study. Future analyses will focus on the association between the observed changes in anthropometric measures and the specified energy balance-related behaviours.

Stratified outcome analyses were interesting. It appeared that the intervention only had an effect on the changes in sum of skinfolds in women and not in men. It would be interesting to see if this is a result of the engagement in different energy balance-related behaviours of men and women.

The present study is one of few obesity prevention programmes, which is primarily aimed at weight gain prevention through changes in both food intake and physical activity and which is directed at the general public. As recently reported by Eiben and Lissner (2006) most prior studies were either primarily interested in preventing cardiovascular diseases and secondly in preventing weight gain or were targeted at specific high-risk groups, such as pregnant and menopausal women (Eiben \& Lissner, 2006). A weight gain prevention programme, which was specifically aimed at weight gain prevention in the general population through changes in both behaviours, is the Pound of Prevention study. This study was however only effective at 1 year post-intervention (Forster et al., 1988). A 3-year exten- 
sion of the study did not reveal any differences in weight gain by treatment group (Jeffery \& French, 1999). The longer term results and effectiveness of the present study can be regarded as unique.

There are a number of limitations of this study. The first concerns the generalizability and is related to the recruitment of companies, as only $9 \%$ of the approached companies were willing to participate. An important reason for companies not to participate in the NHF-NRG In Balance-project proved to be the randomized evaluation design of the programme, implying that companies were not willing to take the risk of being excluded from the intervention (Kwak et al., 2006a). As a result, we were forced to drop the original randomization design of the programme and assign worksites to the experimental and control group based on matching. A second limitation of the present study is the recruitment of participants. Even though the aim of the project was to prevent weight gain in young adults, there was a relatively high response of older and overweight individuals, in line with observations of other studies (Rimm et al., 1995; Baik et al., 2000). The third limitation is related to the absence of a significant difference in weight changes over time between both groups. The required sample size was determined to be large enough to detect a $0.6 \mathrm{~kg}$ weight difference between both groups, based on the average annual weight gain of Dutch young adults (Visscher et al., 2002). However, weight changes observed in the control group were smaller than those expected, with smaller weight change differences between the groups $(0.5 \mathrm{~kg}$ at 12 months). The smaller increase in weight in the control group is most likely a result of measurement effects. The present study probably did not have enough power to detect this difference.

The findings presented here show the effectiveness of the NHF-NRG In Balance-project and supports the usefulness of prevention, especially regarding long-term maintenance. Additionally, it underscores the importance of systematically developing an intervention that contains both individual and environmental components and is directed at both changing physical activity and dietary behaviour. Furthermore, the results support the notion that more attention needs to be given to generating interest in weight management both among worksites as among individuals who are at risk of weight gain. 



\section{CHAPTER 8}

BEHAVIOURAL AND COGNITIVE EFFECTS OF A WORKSITE-BASED WEIGHT GAIN PREVENTION PROGRAMME: THE NHF-NRG IN BALANCE-PROJECT 


\section{ABSTRACT}

The NHF-NRG In Balance-project aimed to prevent weight gain by stimulating the following weight gain preventive behaviours (WGPBs): increasing the frequency and duration of walking and cycling for transport; increasing the physical activity level at work; decreasing portion sizes and reducing the energy density of ingested food during the day. The present paper examined the effectiveness of the project with regard to the specified WGPBs and their corresponding cognitions. A quasi-experimental pre-test-multiple post-test control group design was applied in 12 worksites $(n=570)$. The intervention group ( 6 worksites) received individual and environmental interventions, directed at both physical activity and food intake. The measurements were executed after 12 months (post intervention) and after 24 months (1 year follow-up). The results showed that nearly all WGPBs in the intervention group improved more than in the control group, even though improvements in behaviours were often too small to reach statistical significance. The results also showed that most observed effect sizes remained stable or even increased during the follow-up year. We were unable to explain the intervention effects on the WGPBs by changes in the hypothesised cognitive mediators, as no positive intervention effects were observed for the cognitive factors. In conclusion the findings presented show the effectiveness of the NHF-NRG In Balance-project and supports the usefulness of prevention of weight gain, especially regarding long-term maintenance of small behavioural changes. 


\section{INTRODUCTION}

Despite expanding interest in weight gain prevention (NHS NICE report, 2006), little is known about the effectiveness of weight gain prevention programmes, as few studies have tested interventions that were designed for this purpose (Klem et al. 2000; Hardeman et al. 2000; Wareham et al, 2005). Therefore, the Netherlands Heart Foundation (NHF) initiated the multidisciplinary research programme 'Netherlands Research programme weight Gain prevention' (NHF-NRG), which aims to evaluate three distinct weight gain prevention interventions targeted at different risk groups (Kremers et al., 2005a). The NHF-NRG In Balance-project is one of these weight gain prevention programmes, which is specifically aimed at young adults aged 20-40 years (Kwak et al., 2007b), as young adulthood is recognized as a high-risk period for weight gain (Sherwood et al. 2000; Sheehan et al. 2003). The intervention is worksite-based, because this is a major environmental context for adults in this age group (Dishman et al., 1998) and because worksites provide many opportunities to reinforce the adoption and maintenance of healthy lifestyle behaviours (Kremers et al., 2005a). The project was systematically developed and implemented based on the Intervention Mapping protocol (Bartholomew et al., 2001) and aimed to prevent weight gain by stimulating the most important and relevant weight gain preventive behaviours (WGPBs) in this age group, namely: increasing the frequency and duration of walking and cycling for transport; increasing the physical activity level at work; decreasing portion sizes and reducing the energy density of ingested food during the day (see Kwak et al., 2007b for a comprehensive description of the project development and content). The objective of the intervention was to encourage relatively small behavioural changes, since these changes do not drastically alter current lifestyles (Hill et al., 2003), making them more likely to be sustained over time and to eventually have a large impact on changes in body weight (Seidell, 2000).

In order to promote the specified WGPBs and their determinants the project used a selection of suitable theoretical models and methods of behavioural change (Kwak et al., 2007b). Based on a taxonomy of behaviour change methods for assisting individuals in developing interventions directed at weight gain prevention (Hardeman et al., 2000) and on theoretical input from multiple behaviour change theories, such as the self-regulation theory (Boekaerts et al., 2000; De Ridder \& De Wit., 2006), Precaution Adoption Process Model (Weinstein, 1988), Elaboration Likelihood Model (Petty \& Cacioppo, 1986), Theory of Planned Behaviour (Ajzen, 1991), Social Cognitive Theory (Bandura, 1986) and Habit Theory (Triandis, 1977), several behaviour change methods were applied. These include (self) monitoring, (self) evaluation, personalization of risk, delivering information, tailoring, goal setting, prompting, environmental changes, social encouragement and social support.

An evaluation study of the anthropometric outcomes of the NHF-NRG In Balance-project showed a positive effect of the programme on body composition measures of the individuals in the intervention group (Kwak et al., unpublished data). To gain further insight into effectiveness of the programme it is vital to understand the behavioural and cognitive impact of the programme. The present paper will examine the effectiveness of the NHF-NRG In Balance-project on the specified WGPBs and corresponding cognitions.

\section{METHODS}

\section{The NHF-NRG In Balance-project intervention}

The rationale, theoretical framework, methods, development and implementation 
of the intervention have been described elsewhere (Kwak et al., 2007b). Briefly, the intervention consisted of an individual component and a worksite (environmental) component. The individual component included feedback on measured body composition measures, the "In Balance-box" which contained self-monitoring devices such as a pedometer and waist circumference measuring tape, a website containing general information on the project and on WGPB and two computer-tailored CD-ROMs. The first CD-ROM was directed at awareness of weight status and knowledge regarding the WGPBs, the second at assisting the participants with changing their WGPBs. The environmental components were incorporated into a handbook, which served as a guide for assisting a worksite linkage board (Bartholomew et al., 2002) through the different stages of diffusion of the environmental interventions. Suggested interventions included for example changes in the assortment of food products in the cafeteria, workshops, an information wall containing information on the balance between food intake and physical activity, posters/prompts stimulating stair use and ways to form lunchwalking and cycling groups. Environmental interventions were modified to fit within specific worksites.

\section{Design, recruitment and participants}

The study had a quasi-experimental pre-test-multiple post-test control group design. A sample of 128 eligible worksites was invited to take part in this study, of which twelve (9\%) agreed to participate (Kwak et al., 2006a). Participating worksites employed between 100-800 employees and were located in the south of the Netherlands. The following types of worksites participated; two hospitals, two local governments, five factories (paper-factory, tile-factory, pigment-factory, furnish manufacture and cable-factory), one university, one water-supplying company and one energy-supplying company. The 12 worksites were matched based on the social economic status of their employees and assigned to either the intervention $(n=6)$ or control condition $(n=6)$. Employees within the participating worksites were contacted by letter and invited to participate in a weight gain prevention programme. Eligible participants had a body mass index (BMI) above $18 \mathrm{~kg} / \mathrm{m}^{2}$ and were not under any medical restrictions with regard to diet or physical activity behaviour. An information booklet describing the project in more detail and an informed consent form were sent to interested individuals. All participants read and signed the informed consent form before participating in the study. The Medical Ethical Committee of the Academic Hospital Maastricht, the Netherlands, approved the study protocol.

Worksites and participants in the intervention condition received the NHFNRG In Balance-intervention for a period of 1-year. Individuals in the control group were only contacted for baseline and follow-up measurements. Effect measurements were conducted at baseline, 12 and 24 months. Methods included anthropometrics and a self-administered written questionnaire. Data collection started in September 2003 and was completed for all study participants in August 2006.

\section{Measures}

Socio-demographic characteristics and anthropometrics

The first part of the questionnaire assessed gender, date of birth, marital status (married or living together; separated, divorced, widowed, never married) and highest level of education (high school or less; college/vocational training; university). In the morning, after an overnight fast, body weight $(\mathrm{kg})$ was measured, in underwear, to the nearest $0.1 \mathrm{~kg}$ with a digital laboratory scale and height $(\mathrm{m})$ was measured to the nearest $1.0 \mathrm{~mm}$ without shoes with a mobile measuring unit at the worksite. Additionally, BMI $\left(\mathrm{kg} / \mathrm{m}^{2}\right)$ was calculated. 
Physical activity behaviour

Physical activity behaviours were measured with the validated Dutch short questionnaire to assess health enhancing physical activity (SQUASH; Wendel-Vos et al., 2003). The SQUASH was chosen as it is structured in such a way that it is possible to assess days per week and average time per day spent on daily moderate physical activities: commuting activities (walking and cycling to/from work), physical activities at work (light work (sitting/standing with some walking) and intense work activities (regularly lifting heavy objects at work)), leisure time physical activities (walking, cycling, gardening, odd jobs) and on household activities (light and intense household work). Additionally, the SQUASH assesses time spent on sports. To further assess physical activity at work, two specific items were added to the SQUASH. Participants were asked to indicate how many days a week and minutes a day, they walked during lunch and how many stairs they took per day.

\section{Dietary intake behaviour}

The assessment of dietary intake focused on fibre intake, energy density and portion sizes (72 items). Food intake was measured using the validated 'ENVET'-list (Camps \& Abden, 1994). Questions regarding fibre, fruit and vegetable intake were added to the questionnaire (Department of Human Nutrition, WU). Additionally, seven items were added to the questionnaire to assess portion sizes.

\section{Fibre intake}

The consumption of fibre-rich and fibre-poor products was assessed for bread meals and main meals. Participants indicated either per day, week or month how many servings they consumed. Fibre-rich bread was assessed with one item; participants indicated how many slices of whole grain bread they consumed. Two items were used to measure the consumption of breads classified as being low in fibre (white bread and wheat bread). For the consumption of fibre-rich products during the main meal five items were used, participants indicated how often they consumed the following products: whole grain pasta, whole grain rice, pulses, salad or raw vegetables and boiled, steamed or baked vegetables. Two items were used to measure the consumption of fibre-poor products (ordinary pasta and white-rice) during the main meal.

\section{Energy density}

The consumption of products with a low energy density and high energy density was measured for bread toppings, main meal, snacks and beverages. The measurement of low energy dense bread toppings was assessed with three items; participants indicated how many slices of bread they ate with: low fat cheese, lean meat and low energy dense sweet toppings. The intake of high energy dense bread toppings was measured by assessing the intake of the high energy denseversion of these products ( 3 items). For the consumption of low energy dense products during the main meal three items were used. Participants indicated how often they consumed the following products: lean meats (e.g. chicken without skin), light dressings and low fat yoghurt. The consumption of high energy dense products during the main meal was measured with ten items (fries, Chinese/pizza, fatty meats (sausages, mince etc), gravy, cheeses, mayonnaise, crème fraîche and full fat yoghurt). Three items were used to assess the consumption of snacks with a low energy density; participants indicated how often they consumed small biscuits, Japanese Mix (small savouries) and fruit. The number of servings of fruit was assessed with two items, one related to citrus fruit and the other to remaining fruits. The consumption of snacks with a high energy density was measured with eleven items (pastries, tarts, chocolates, 
chocolate bars, peanuts, crisps, crackers with cheese/pate, savouries, pizza, creamy salads and herring). The intake of drinks with a low energy density was assessed by asking participants how many units of skimmed milk, semi-skimmed milk, mineral water and light beverages they consumed. Four items were used for the consumption of high-energy dense drinks (full fat milk, sweetened beverages, sweetened fruit drinks and alcohol). For both beverage categories a total score was calculated by transforming the units into millilitres.

\section{Portion sizes}

Portion size was assessed with seven items in total, for chocolate bars, butter, sweet bread toppings, and for cheese and meat bread toppings. Participants were asked to indicate on a 4-point scale which portion size they usually consumed, for example 'I don't eat chocolate bars' to 'super size' and 'I don't eat cheese on bread' to 'my bread is fully covered with several slices of cheese'. The four items related to bread toppings were summed into one score. Additionally, participants were asked if they usually finish their entire meal and if they ask for second helpings in the company canteen (5-point scale 'never' to 'always').

\section{Motivational stage}

Motivational stages in the adoption of behaviour were assessed for each WGPB, based on the Precaution Adoption Process Model (PAPM; Weinstein 1988). The items were phrased as follows: 'Which of the following statements best describes you? (Tick only one answer)'. The answering categories, with regard to eating smaller portions, for example, were stated as follows: 'I have never thought about eating smaller portions' (unengaged); 'I have thought about it, but don't know if I am going to eat smaller portions' (undecided); 'I have decided not to eat smaller portions' (decided not to act); 'I have decided to eat smaller portions in the future'(decided to act); 'I already eat smaller portions, but have started in the last 6 months' (action); 'I have been eating smaller portions for more than 6 months' (maintenance).

\section{Cognitive determinants of WGPBs}

In order to examine the intervention effects on the cognitive determinants of the specified WGPBs, items based on the Theory of Planned Behaviour (TPB; Ajzen, 1991) were included in the questionnaire. The items assessed intention, attitude, social influences, and perceived behavioural control.

\section{Intention}

The intentions towards engaging in each of the WGPBs were each assessed with single items, asking participants to indicate whether they intended to perform the behaviour more often within the next six months (5 point-scale 'certainly not' (1) to ‘certainly yes' (5)).

\section{Attitude, subjective norm and perceived behavioural control}

Attitudes, subjective norm and perceived behavioural control were assessed with respect to preventing weight gain. The concept of preventing weight gain was conceptualized into the term 'watching one's weight' (translation of a popular Dutch expression regarding preventing weight gain; Wammes et al., 2007). Attitude toward 'watching one's weight' was assessed with two items ('When I watch my weight, I think this is good' and 'When I watch my weight, I think this is pleasant'), which were answered on a 5-point scale ('bad' to 'good' and 'unpleasant' to 'pleasant'). The items were included in further analyses separately because of their low internal consistency. To assess subjective norm three items were used, participants indicated 
on a 5-point scale ('totally disagree' (1) to 'totally agree' (5)) if their [partner, friends, colleagues] thinks that they should watch their weight (Cronbach's $\alpha=0.80$ ). The scale for perceived behavioural control consisted of 8 items. Participants indicated on a 5-point scale ('absolutely unable' (1) to 'absolutely able' (5)), how confident they were about their ability to watch their weight under difficult circumstances, such as during parties, when they are busy or when they are tired (Cronbach's $\alpha=0.82$ ).

\section{Process evaluation}

The two post-test questionnaires for the participants of the intervention group included an additional section with process questions evaluating the intervention. Questions were related to participation, comprehension and satisfaction of the various intervention components. Participants were asked how often they had used the materials (e.g. read the personalized feedback, used the pedometer, and visited the website and which part of the personalized advice they followed). Additionally, participants indicated whether the materials were comprehensible (5point scale: 'very unclear' (1) to 'very clear' (5)) and interesting (5-point scale: 'very uninteresting' (1) to 'very interesting' (5)). Further, participants were asked to give an overall rating of each specific intervention component, including specific parts of the website and computer tailoring, on a scale from 1-10. Specific items about the environmental interventions assessed participation (e.g. how often do you walk during lunch as a result of the 'Lunch walking' campaign?), and satisfaction (rating on a scale from 1-10).

To ascertain the extent to which the environmental interventions were implemented within the worksites the research team executed observations within each worksite. Additionally, the reach of specific environmental interventions was measured by registration of activities, materials distributed and participation rates. The same researcher executed the observations during the two-year data collection period.

\section{Statistical analyses}

To assess potential selective dropout at both follow-ups, multiple logistic regression analyses were conducted with dropout as dependent variable and the baseline characteristics age, gender, BMI, marital status, education and smoking status as independent variables.

Descriptive statistics were performed to describe demographic characteristics (age, gender, BMI, marital status, education and smoking status), the WGPBs and their corresponding cognitions at baseline. The effect analyses were performed in 4 steps. First, student's t-tests and Chi-square tests were used to explore baseline differences between participants in the intervention and control group. Second, differences between the intervention and control group in changes in moderate intensity physical activities, active commuting to work, physical activities at work, household activities, leisure time activities, dietary fibre intake, intake of energy dense foods, portion sizes at 12 and 24 months were examined using linear regression analyses. Adjusted for the baseline value of the particular variable as well as group membership and various baseline characteristics (age, gender, BMI, marital status, education and smoking status), with $B$ representing the intervention effect. Cohen's $d$ effect sizes were calculated in order to assess the magnitude of the intervention effect; $d$ is defined as the difference between two means divided by the pooled standard deviation for those means (Cohen, 1988). The interpretation of Cohen's $d$ effect sizes was done according to Lipsey's (1990) guidelines; a small effect size is equal or smaller than 0.32 , a medium effect $0.33<$ Cohen's $d \leq 0.55$, and a large effect when larger than 0.56 (Lipsey, 1990). Third, differences between the groups 
for precaution adoption stage progression were assessed with a logistic regression. Fourth, the intervention impact on the hypothesized cognitive mediators was assessed with linear regressions, applying the same protocol as presented for the behavioural outcomes.

As a consequence of the study design intervention effects were expected to be nested within worksites. It was expected that a high probability of interdependence would occur between participants of the same worksite. To take this into account, all multilevel linear regression analyses were conducted in MlwiN with random intercepts (Rabash et al., 1999). A 5\% level of significance was used.

\section{RESULTS}

\section{Response rate analyses}

At baseline, the average response rate to the questionnaire was $88.1 \%$ (487/553). At the first follow-up the response rate average was $82.5 \%(\mathrm{n}=376)$ and at the second follow-up 75.8\% ( $\mathrm{n}=303)$. During the course of the project, one of the worksites in the intervention group underwent a reorganisation; half of the employees were made redundant, thereby causing most of the drop-out in our study. There was no selective dropout at the first follow-up. At the second follow-up smokers were more likely to discontinue the study than non-smokers (Odds Ratio $(\mathrm{OR})=2.33,95 \%$ confidence interval (C.I.) 1.39-4.06).

\section{Baseline characteristics of the respondents}

Demographic characteristics regarding gender, marital status, education and ethnicity were similar in the intervention and control group. Overall, the sample had an equal distribution of men and women (53.4\% female), and of individuals with a high or low educational level (57.2\% higher education). The sample was predominately married or living together $(81.5 \%)$. The groups did differ significantly regarding age and BMI. Participants from the intervention group were significantly older (38.5 \pm 7.9 years) than participants from the control group ( $34.8 \pm 7.1$ years; $\mathrm{p}<.001$ ) and had a slightly higher BMI $\left(25.6 \pm 3.8 \mathrm{~kg} / \mathrm{m}^{2}\right.$ and $24.2 \pm 3.1 \mathrm{~kg} / \mathrm{m}^{2}$ respectively; $\left.\mathrm{p}<.001\right)$.

\section{Physical activity behaviours}

The mean scores of physical activity behaviours at baseline and the changes identified at the 12 and 24 months are presented in Table 1 . The average time spent on moderate intensity physical activities differed significantly between the two groups at baseline, with individuals in the control group spending more time on moderate intensity activities, as a result of time spent on physical activities at work. Individuals from the intervention group however spent more time per week on active commuting and household activities than individuals from the control group.

After 12 months individuals in the intervention group increased their moderate intensity physical activity significantly more than individuals from the control group (Cohen's $d=0.27$ ), decreasing slightly at 24 months (Cohen's $d=0.25$ ). Except for a smaller increase in active commuting to work, the intervention group reported greater increases in all other moderate intensity physical activities in comparison to the control group at 12 months, with household activities largely responsible for the difference between groups. 


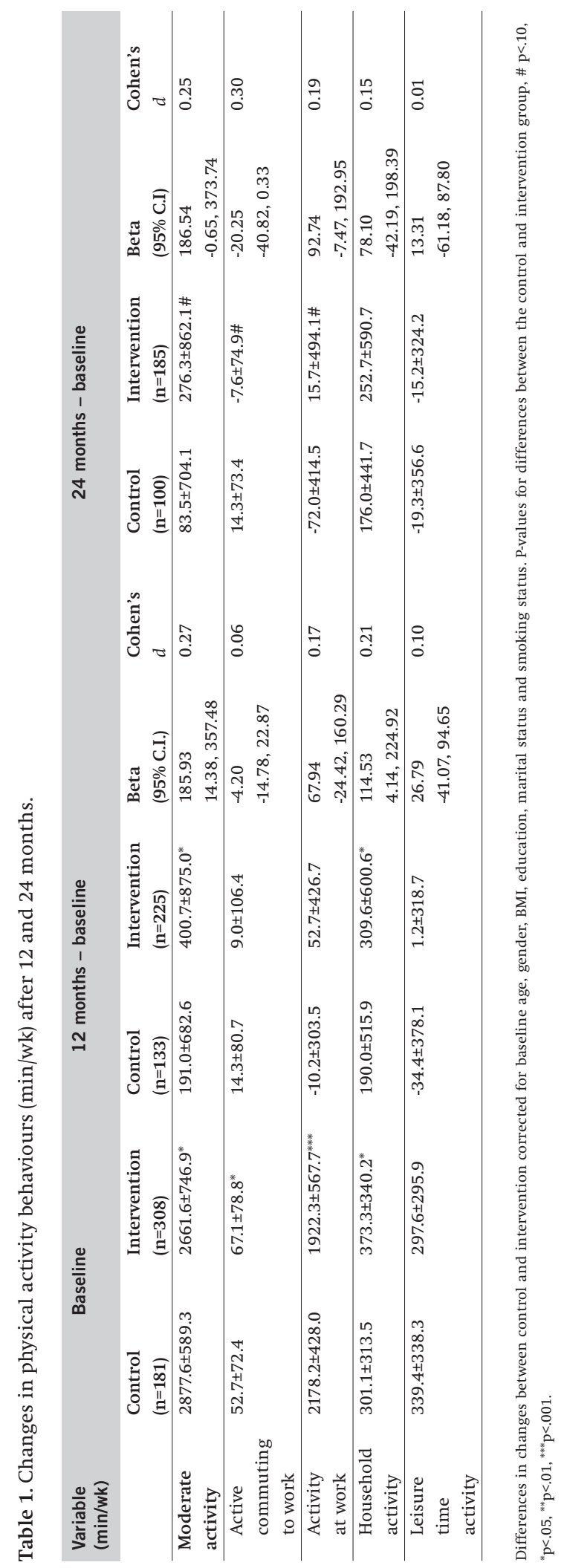




\section{DIETARY BEHAVIOURS}

Table 2 describes the mean scores of the dietary related behaviours of the WGPBs at baseline and the effects of the project on these behaviours at 12 and 24 months. No significant difference was observed between the intervention and control group regarding fibre intake at baseline. At 12 months individuals in the intervention group reported a decrease in the consumption of low-fibre bread, and at 24 months they further decreased their consumption which was significantly different from the increase reported by the control group (Cohen's $d=0.31$ ).

Baseline data regarding the intake of energy dense food during the day only revealed a significant difference between the intervention and control group for the consumption of low energy dense products consumed during the main meal, which was higher in the intervention group. At 12 months individuals in the intervention group reported a decrease in consumption of high energy dense bread toppings, which was significantly different from the increase reported by the control group (Cohen's $d=0.41$ ). The effect further increased after 24 months (Cohen's $d=0.43$ ). The intervention also had a medium effect (Cohen's $d=0.37$ ) on the increased consumption of low energy dense products during the main meal at 24 months. An increase was reported by individuals in the intervention group which differed significantly from the decrease reported by the control group. No significant intervention effects were observed for the changes in portion sizes. 


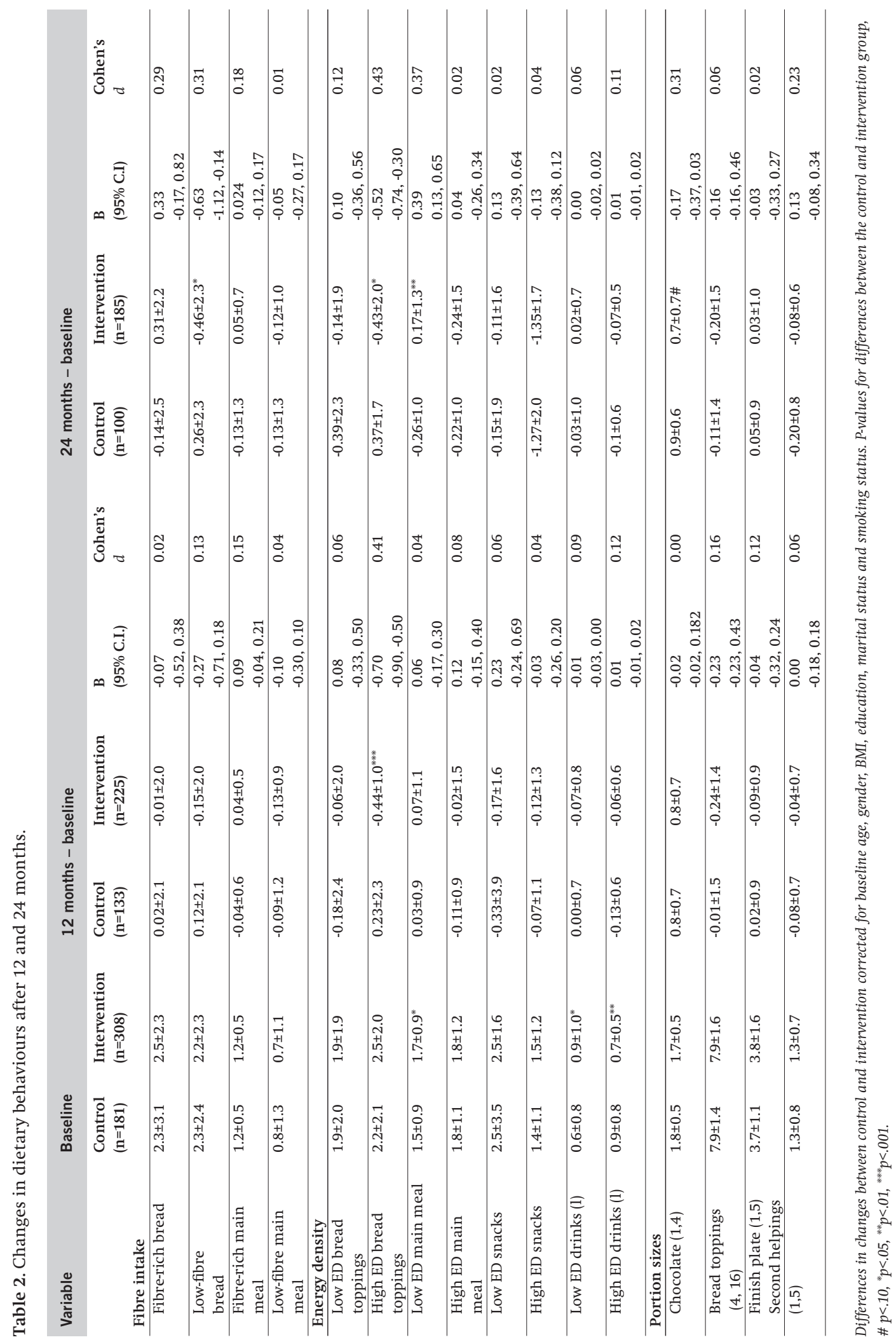




\section{Precaution adoption phases}

The distribution of individuals over the 6 stages differed significantly between the intervention and control group at baseline for all the WGPBs (Table 3). Individuals in the intervention group resided in stages closer to engagement in WGPBs, compared to individuals from the control group. Table 3 also shows the Odds Ratios (ORs) for each WGPB with regard to forward stage transition from baseline to the stage at 12 and 24 months follow-up, respectively. Generally, the ORs show a favourable stage transition in the intervention group, compared to the control group (all ORs > 1, except for eating less calories at 12 months). The OR that compared the intervention and control group with regard to stage progression towards active commuting to work at 12 months was the only OR that revealed a statistically significant difference (95\% C.I. 1.038, 2.733), though.

\section{Cognitive factors}

Intentions towards engaging in the WGPBs differed significantly between the control and intervention group at baseline (Table 4). Individuals in the intervention group had significantly greater intentions to engage in the WGPBs than individuals in the control group. In contrast to our hypothesis at 12 and 24 months individuals in the intervention group reported a significantly greater decrease in their intention toward eating fewer calories in comparison to individuals in the control group (Cohen's $d=0.26$ and 0.30 respectively). This difference was also observed for the intention toward eating smaller portions at 24 months (Cohen's $d=0.24$ ). With respect to subjective norm individuals in the intervention group perceived a more positive subjective norm among partners, friends and colleagues towards watching one's weight than individuals from the control group at baseline.

At 12 months individuals in the intervention reported a decrease in subjective norm towards watching one's weight in comparison to an increase in the control group (Cohen's $d=0.25$ ).

No significant impact of the project was observed on changes in attitudes or perceived behavioural control neither at 12 or 24 months. 


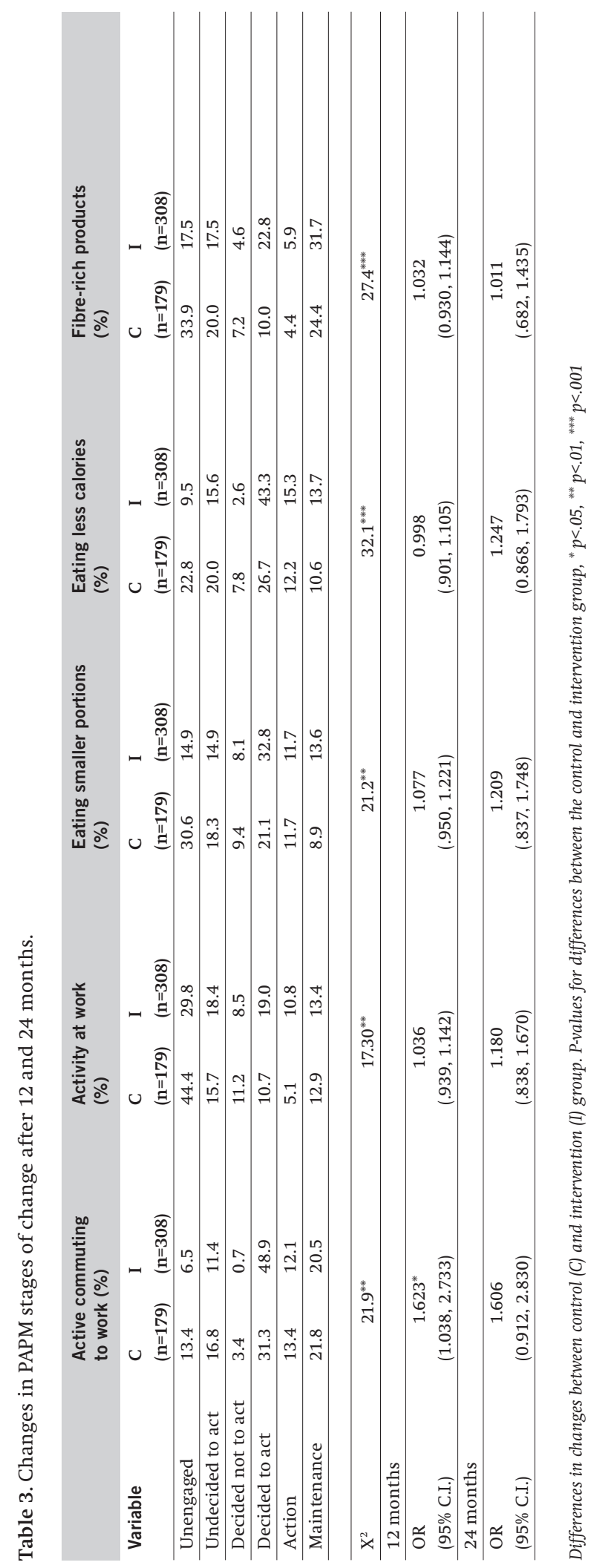




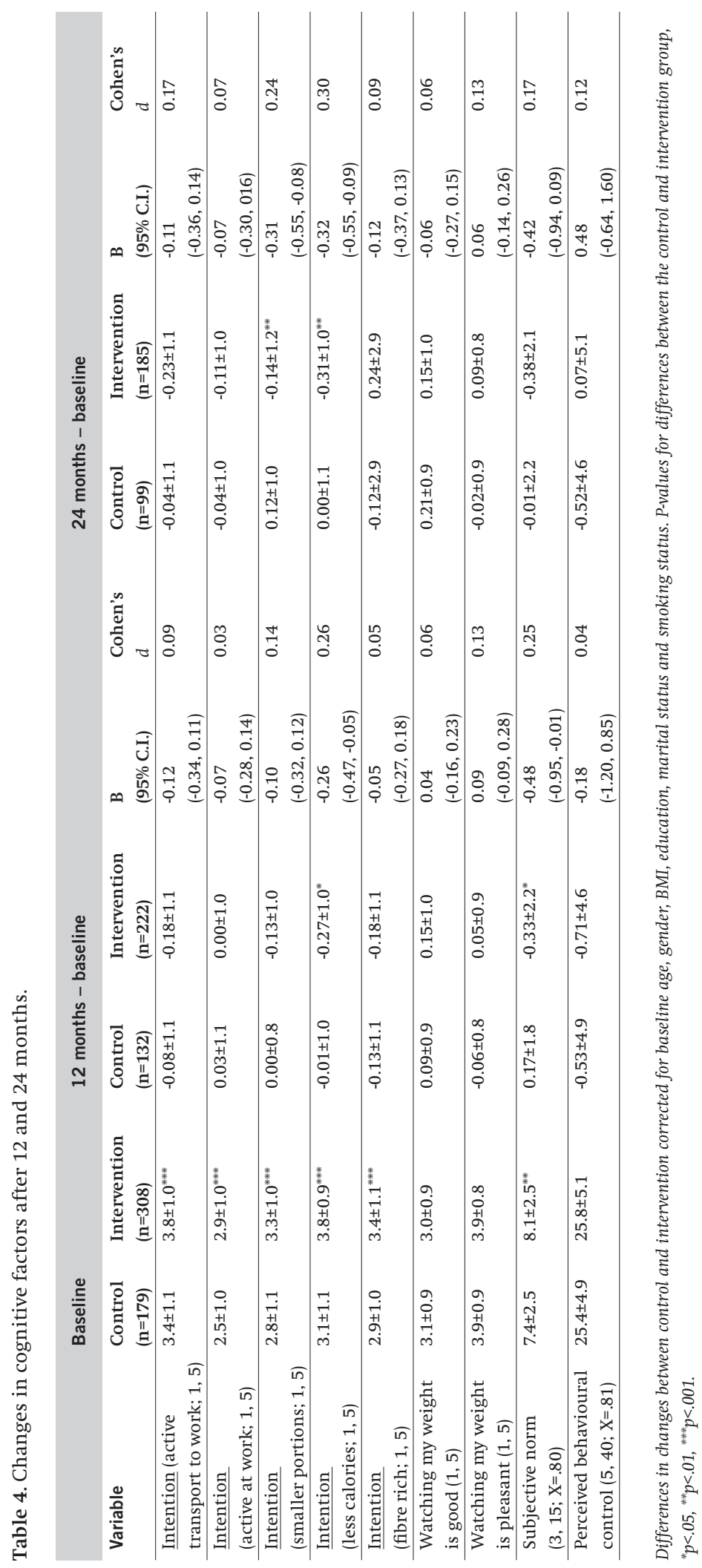




\section{Process evaluation}

The results of the process evaluation are presented in table 5 and only represent the participants completing the process questions for both the first and second followup. Response to most of these questions was low, (mean response $41.6 \%$; range $7 \%$ and 98\%). Almost all participants indicated that the personal feedback on measured body composition measures was very interesting and comprehensible. Results revealed that more than half of the participants found the intervention materials interesting and more than $75 \%$ found them comprehensible. The personal feedback was reported to be most interesting and comprehensible. The 'Energy Balance' tailoring programme was found least interesting (58.2\%). Almost all participants (87.4\%) reported that they thoroughly read the personal feedback on body composition measures. Of all materials that made up the In Balance-box the pedometer was used most often; both after the first and second follow-up. The introduction brochure and the calorie guide were most appreciated. Nearly $72 \%$ of participants reported visiting the website during the first year of the intervention, this dropped to $16.8 \%$ during the second follow-up. During the first follow-up participants indicated that of the information present on the website all topics were read as frequently, with an average rating of 7.2. During the second follow-up participants indicated to have mostly read the information on physical activity, which also received the highest rating. The tailoring programme focussing on general information regarding energy balance was used 1.4 times during the first follow-up, this dropped to 0.4 during the second follow-up. Of the participants $21.4 \%$ indicated to have carried out the personal advice given, in comparison to $10.7 \%$ during the second follow-up. The physical activity part of the second tailoring programme was used most often, both during the first and second follow-up. The advice given was carried out by $29.1 \%$ (follow-up 1), dropping to 6.6\% during the second follow-up.

The intervention directed at stimulating participants to take the stairs was most appreciated and had the largest impact on the intended behaviour. More than half of the participants who responded to these questions during the first follow-up indicated that they took the stairs more often as a result of this intervention, on average 5.6 times more a day. During the second follow-up, still half of the participants indicated to take the stairs more often, with an average of 4.1 times a day. The bike campaign appeared to have least impact; only few participants ( $5 \%$ follow-up 1 , $9.2 \%$ follow-up) indicated that they took the bike as a result of the campaign.

Data collected by observation and registration of activities revealed that of the six intervention worksites only two formed a worksite linkage board. The other four worksites declined, stating time investment problems as their main reason. However, four worksites still implemented environmental interventions, such as placing articles in the personnel magazine regarding the project, handing out free apples during National Health Week, distributing brochures, waist measuring tapes, maps and walking routes, placing posters near the elevators and stairs to stimulate stair use and organizing a workshop on healthy eating given by a dietician. 


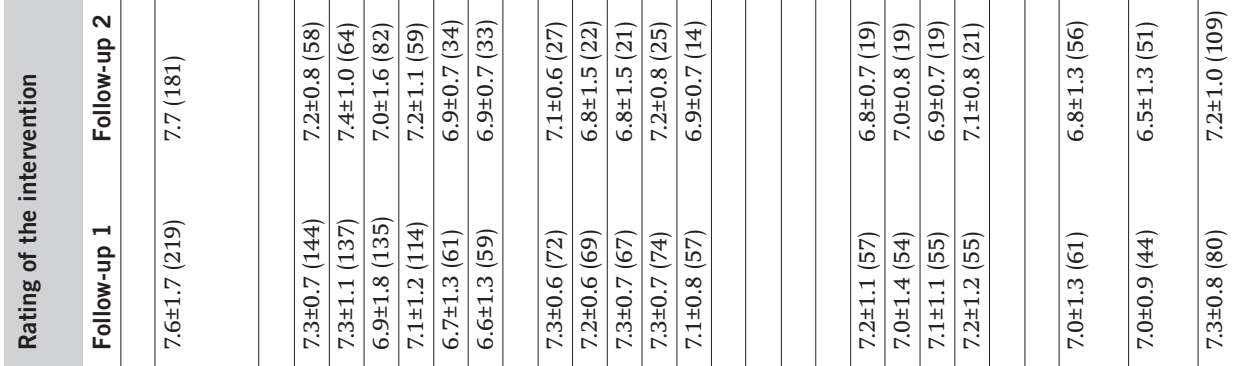

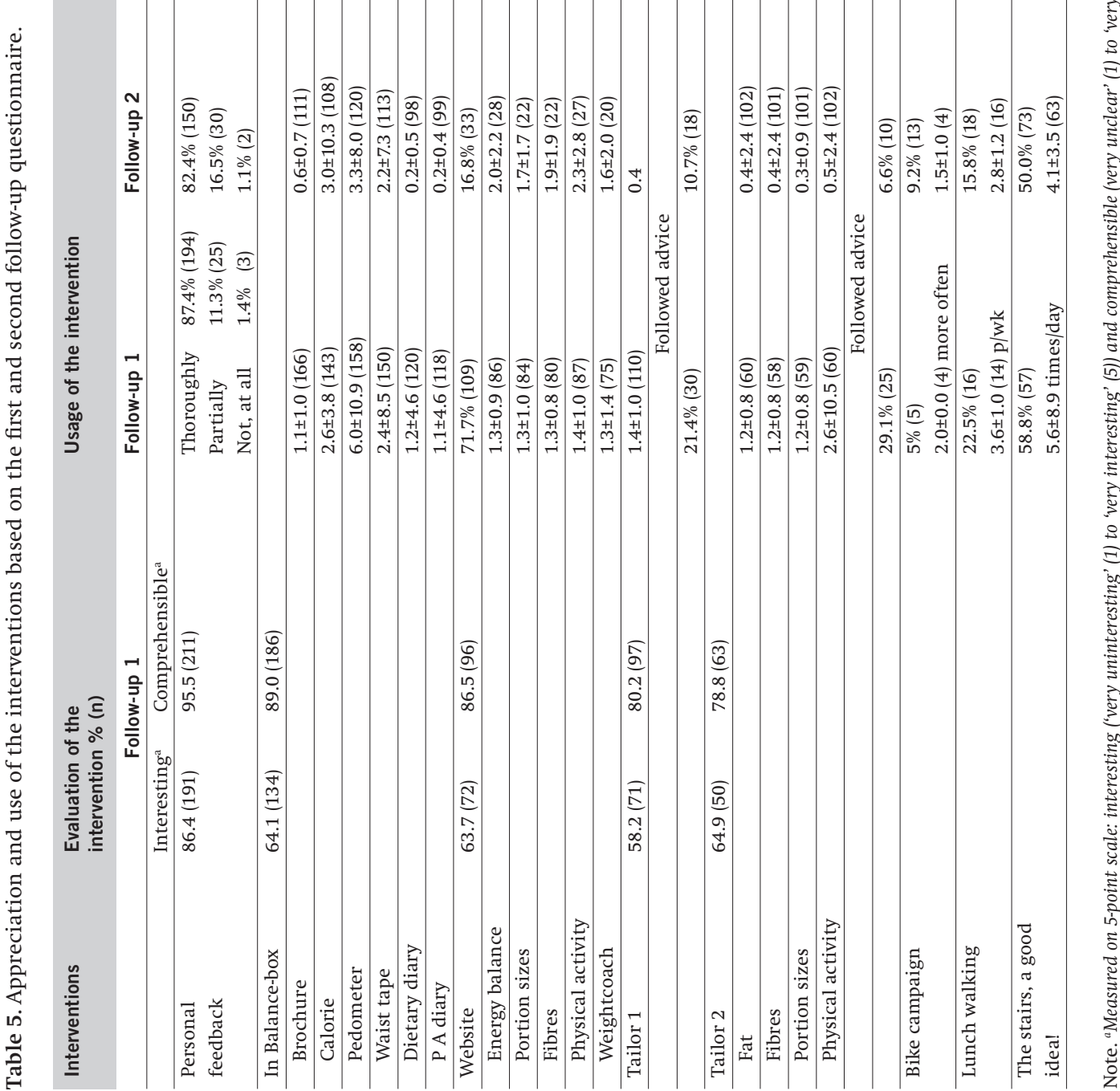




\section{DISCUSSION}

The results of the present effect evaluation of the NHF-NRG In Balance intervention indicate that nearly all dietary intake and physical activity behaviours in the intervention group improved more than in the control group, even though improvements in behaviours were often too small to reach statistical significance. The small to medium magnitude of the effects was in line with the objective of the study to stimulate relatively small behavioural changes and are highly relevant as the summed changes are related to relevant improvements in sum of skinfolds and waist circumferences as observed in an earlier study (Kwak et al., submitted; Chapter 7). Moreover, the results supported the assumption that small behavioural changes are more likely to be sustainable (Hill et al., 2006), as most observed effect sizes remained stable or even increased over time. Incorporating these small behavioural changes into everyday life routines thus seems likely to facilitate permanent adoption of these behaviours.

Results revealed that at 12 months individuals in the intervention group were on average 1.6 times more likely than individuals from the control group to have progressed in motivational stage towards active commuting to work. However, we did not observe effects on this specific WGPB within the behavioural data. The results of the process evaluation showed that more than half of the participants found the intervention materials interesting and more than $75 \%$ found them comprehensible, the personal feedback was reported to be most interesting and comprehensible, the 'Energy Balance' tailoring programme least interesting. Despite, that only two worksites implemented a worksite linkage-board, four worksites implemented environmental interventions.

We were unable to explain the intervention effects on the WGPBs by changes in the hypothesised cognitive mediators, as no positive intervention effects were observed for the cognitive factors. However, this finding does correspond with findings from previous intervention studies, which have also observed changes in dietary or physical activity behaviours without first observing changes in the corresponding cognitive factors (Eriksen et al., 2003; Perry et al., 2004; Jurg et al., 2006). This would imply that especially our environmental interventions may have had a direct effect on behaviour, thus unmediated by the cognitive mediators as theorized in the TPB (Ajzen, 1991). In a recent observational study (De Bruijn et al., 2005), similar results have been found with respect to energy balance-related behaviours in adolescents. The direct influence of the environment on behaviour may reflect a merely automatic, unconscious process, which is largely unmediated by cognitive factors (Kremers et al., 2006). This further emphasizes the importance of not just intervening by health education activities on changing the cognitive factors in order to change WGPBs, but to include changes in the so-called obesogenic environment as done in the present study.

An alternative explanation for the lack of positive intervention effects on cognitive factors is methodological. In order to keep the questionnaire from becoming too long and risk low response rates and invalid data, the number of items used to assess cognitive factors was kept to a minimum (Kremers et al., 2005d). Instead of assessing the cognitive factors for each specified WGPB the questions were framed in relation to 'watching one's weight'. Nevertheless, individuals might not associate the WGPBs with watching their weight, but associate them with for example an overall healthy lifestyle. However, we did assess intentions towards engaging in the WGPBs with behaviour-specific items. The absence of positive intervention effects for changes in intention indicates a low likelihood of a considerable intervention impact on the other TPB variables. 
Several limitations of the present study should be noted. The first limitation pertains to not all changes in WGPBs being statistically significant, which can be explained by our aim to stimulate relatively small changes, which are difficult to measure, additionally individuals could choose to change one WGPB. Firstly, the study relied on the use of self-report measures, which may be subject to challenges of memory, estimation and bias (NIH guide, 2002). Additionally, self-report measures are prone to both over-reporting and under-reporting (Bandini et al., 1990; Schoeller, 1990). The results regarding the WGPBs should therefore be interpreted with caution, even though validated questionnaires were used. A second limitation pertains to the external validity of the results, both with regard to worksites and individual participants. Worksites that are enrolled in studies such as the present are most likely more interested in health promotion than the average worksite (Sorensen et al., 1996), resulting in a selection bias. Moreover, only $9 \%$ of the approached companies were willing to participate in the NHF-NRG In Balance-project. An important reason for worksites to decline participation proved to be the randomized evaluation design of the programme. As a result, we did not randomize worksites, but assigned them to the experimental and control group based on matching. The fact that we were unable to apply a randomized study design (Kwak et al., 2006a) is likely to have contributed to this bias. A second limitation of the present study is the recruitment of participants. Baseline results revealed that individuals from the intervention group were more motivated to change than individuals in the control group. Moreover, there was a relatively high response of older and overweight individuals, even though the project was aimed at weight gain prevention in young adults. This is in line with observations of other studies (Rimm et al., 1995; Baik et al., 2000). A final limitation concerns the process evaluation. Data collected during the process evaluation indicated that the response to the process questions was very low, making this data inappropriate for use towards the effectiveness of NHF-NRG In Balance. The low response could either be a result of the length of the questionnaire, as these questions were placed at the end, or this could indicate that participants were dissatisfied with the intervention.

In conclusion, the NHF-NRG In Balance-project resulted in small to medium effects in nearly all dietary intake and physical active behaviours. As the incorporation of these relatively small behavioural changes into everyday life routines are likely to facilitate permanent adoption of these behaviours they may be regarded as of potential public health significance. Additionally, it underscores the importance of systematically developing an intervention that contains both individual and environmental components. 
GENERAL
DISCUSSION 
This thesis reports on the development, implementation and evaluation of the NHF-NRG In Balance-project, a worksite-based prevention programme directed at the prevention of weight gain in young adults, through changes in both physical activity and food intake. The overall aim of the thesis was to test the effectiveness of the programme. Several studies were conducted to reach the overall aim. First, a stepwise theory and evidence-driven intervention programme was developed based on the Intervention Mapping protocol. The next chapter elaborated on the recruitment problems that occurred before implementation of the programme. The third chapter explored the accuracy of the physical activity questionnaire that was used to evaluate one of the outcome measures, by comparing it with a 24 -h recall and an accelerometer. Furthermore three studies were conducted to test the effectiveness of one of the intervention components of the programme, namely promoting stair use. The last two studies, examined the short-term and longer term effectiveness of the programme, with regard to changes in anthropometric measures, weight gain preventive behaviours, motivational stages and corresponding cognitive factors.

The purpose of this final chapter is to summarize and integrate the main findings of the studies outlined in this thesis and to discuss methodological and intervention related issues, as well as implications for practice and future research.

\section{MAIN FINDINGS}

\section{Development, implementation and evaluation framework}

In order to ensure that the programme was based on a foundation of theoretical, empirical and practical information, Intervention Mapping (IM) was used to guide the development, implementation and evaluation of the programme (General Introduction). Applying the IM protocol to the development of the NHF-NRG In Balanceproject, resulted in a stepwise multi-component intervention programme, which was directed at raising awareness and at motivating and enabling the following weight gain preventive behaviours (WGPBs): increasing frequency and duration of walking and cycling to work, (2) increasing physical activity level at work, (3) decreasing portion sizes and (4) decreasing intake of energy-dense foods. Participants receiving the programme were given the opportunity to choose the WGPBs that they would like to change (General Introduction). It has been suggested that giving participants this opportunity will more likely lead to behaviour change (Kremers et al., 2005a). Additionally, it has been shown that clustering occurs both within energy balance-related behaviours as within their cognitive determinants, suggesting a synergistic cognitive motivational mechanism within these behaviours (Kremers et al., 2004; De Vries et al., in press.). The programme consisted of both individual interventions, i.e. feedback on body composition measures, self-monitoring devices such as a pedometer, a website and computer tailoring, as worksite (environmental) interventions, i.e. changes in the assortment of food products in the cafeteria, workshops, posters/prompts stimulating stair use, ways to form lunch-walking and cycling groups. The environmental interventions were incorporated into a handbook, which served as a guide for assisting a worksite linkage board through the different stages of diffusion of the environmental interventions. During the application of the IM protocol it became apparent that creating learning and change objectives for all the identified cognitive and environmental determinants of the several weight gain preventive behaviours (WGPBs), as proposed by IM, would be very impractical. In order to restrict the amount and complexity of matrices, it was decided to guide the specification of the performance objectives according to self-regulation theory (Boekaerts et al., 2000) and implementation intention theory (Gollwitzer, 1999). 
In order to determine the efficacy of the programme an evaluation plan was developed, in which twelve worksites would be randomly assigned to the intervention and control group. However, the recruitment of worksites proved to be problematic (Chapter 2). Of the 128 worksites that were approached for participation, only 12 companies $(9 \%)$ were willing to participate. The most important reasons for refusal to participate were lack of time and resources in times of economic stagnation and unwillingness to be subjected to the demands of research, such as randomisation. As a result, the randomization design of the programme could not be applied, and a quasi-experimental design was used instead.

The developed evaluation plan of the programme included a process and effect evaluation, which entailed the assessment of several outcome measures. Chapter 3 reports on a study conducted to explore the accuracy of the questionnaire used to assess the outcome measure of the physical activity related WGPBs. The study compared the questionnaire, a 24-h recall and an accelerometer. Results of this study showed that the questionnaire had practical advantages in terms of convenience of administrating, but its usefulness in estimating physical activity levels was limited.

\section{Promoting stair use within the NHF-NRG In Balance-project}

Three studies explored the effectiveness of two interventions on promoting stair use, a useful means to increase lifestyle physical activity.

Based on Gollwitzer's (1999) implementation intention theory, chapters 4 and 5 described two studies which investigated the effectiveness of an intervention using implementation intentions specifying stair use in promoting this behaviour in a worksite setting. The results in chapter 4 showed that, based on observational data, individuals who specified when and where they would take the stairs were more likely to perform this behaviour immediately after forming an implementation intention than those who had formed an unrelated implementation intention. The long-term effectiveness of implementation intentions is relatively unexplored. Only three previous studies have investigated the effectiveness of the formation of implementation intentions over a period of more than two months (Jackson et al., 2005; Prestwich et al., 2005; Sheeran \& Orbell, 2000). We were therefore interested in testing the long-term effectiveness of the implementation intentions formed in Chapter 4. Chapter 5 explored whether the implementation intentions were still effective after 12 months. Self-report results showed that individuals who had formed implementation intentions were more likely to take the stairs 12 months later than those who formed an unrelated implementation intention. However, in line with theory, this was only true for individuals who were motivated at baseline to be more active at work.

Chapter 6 reported on a study investigating the effectiveness of an intervention using prompts on posters, in two types of worksites; one consisting mainly of white-collar workers and one mainly of blue-collar workers. Although previous studies have generally shown the effectiveness of prompts to promote stair use in commercial setting (e.g. Brownell et al., 1980; Kerr et al., 2000, Webb \& Eves, 2005) and white-collar worksites (Kerr et al., 2004; Titze et al., 2001; Vanden Auwelee et al., 2005), no studies have tested the effectiveness in blue-collar worksites. The results showed that overall prompts had a short-term effect in increasing stair use of approximately $5 \%$, which is in line with previous studies that have found similar short-term effects varying from 1\% (Marshall et al., 2002) to 8\% (Vanden Auwelee et al., 2005). The prompts were found to be equally effective in both types of worksites. The intervention was implemented in four of the six worksites participating in the NHF-NRG In Balance-project. The evaluation presented in Chapter 6 is based on data collected in two of these worksites. 


\section{Evaluation of the NHF-NRG In Balance-project}

The last two studies of this thesis examined the effectiveness of the project with regard to short- and longer term changes in anthropometric measures, WGPBs, motivational stages and corresponding cognitions. Chapter 7 focused on the effectiveness with regard to changes in sum of skinfolds, waist circumference, body weight and body mass index (BMI). Baseline differences between the intervention and control group were observed with respect to most of the outcome indicators, probably as a result of the recruitment procedure. The results indicated that with regard to changes in sum of skinfolds and waist circumference the intervention programme was indeed effective both at 12 and 24 months, with effect sizes implying effects of medium magnitude. Overall, the intervention had a positive effect on the body composition measures of the individuals in the intervention group. In the final chapter the effectiveness of the WGPBs, motivation to change and corresponding cognitions was examined. Findings indicated that the intervention improved nearly all dietary intake and physical activity behaviours in the intervention group, in comparison to the control group, even though improvements in behaviours were often too small to reach statistical significance. The small to medium magnitude of the effects was in line with the objective of the study to stimulate relatively small behavioural changes and are highly relevant as the summed changes are most likely related to relevant improvements in sum of skinfolds and waist circumferences as observed in Chapter 7. Moreover, the results supported the assumption that small behavioural changes are more likely to be sustainable (Hill et al., 2006), as most observed effect sizes remained stable or even increased after the one-year follow-up period. We were, however, unable to explain the intervention effects on the WGPBs by changes in the hypothesised cognitive mediators, as no positive intervention effects were observed for the cognitive factors. This would imply that especially our environmental interventions may have had a direct effect on behaviour, thus unmediated by the cognitive mediators as theorized in the TPB (Ajzen, 1991). This further emphasizes the importance of not just intervening on intrapersonal social-cognitive factors in order to change WGPBs, but to include environmental interventions in the prevention of weight gain.

\section{METHODOLOGICAL ISSUES}

The results presented in the different studies throughout this thesis should be interpreted in the light of several methodological strengths and concerns. The methodological issues concern (1) the modified application of the IM protocol, (2) the sample used, (3) the study design employed, (4) the measurement instruments used and (5) the process evaluation.

\section{Application of the Intervention Mapping protocol}

In general, the application of the intervention mapping protocol has provided a systematic approach for the development, implementation and evaluation of the intervention, as a result of which it is based on a foundation of theoretical, empirical and practical information. However, we did not create matrixes of proximal programme objectives by crossing the performance objectives with their determinants as proposed in the IM protocol (Bartholomew et al., 2001). Instead, based on self-regulation theory (Boekaerst et al., 2000) and the implementation intention theory (Gollwizter, 1999), ten specific personal performance objectives were formed for each WGPB, as well as four specific environmental performance objectives. Accordingly, cognitive and environmental determinants were identified for each of 
these performance objectives. We then proceeded to the selection of theoretical methods and practical strategies for the identified determinants, instead of selecting them for the proximal performance objectives as proposed by the original protocol. The rest of the steps were applied in the manner as proposed by IM. We feel that the content of the selected strategies that have resulted from this process is theoretically sound and linked to the objectives that were specified, eventually leading to a theory-and evidence-based intervention This modification has also been made in other interventions that applied the IM protocol to complex behaviours with satisfactory results (Martens et al., 2006; Perez Rodrigo et al., 2005; Singh et al., 2006; Werkman et al., 2006).

Applying the IM-protocol to the development, implementation and evaluation of the programme is a major strength of this study, not only because of its systematic approach which has lead to an intervention based on theoretical, empirical and practical information, but also because very few examples of theory-driven and systematically developed weight gain prevention interventions for adults have been described in the literature

\section{Recruitment of worksites and participants}

For the study conducted in this thesis two levels of participation can be distinguished, a worksite level and an employee level. With regard to both participation levels issues need to be discussed that might have threatened the external validity of the results. Firstly, as discussed in Chapter 2 the worksite participation rate was very low; only $9 \%$ of the approached worksites were willing to participate. This finding is in line with other recent Dutch worksite based health promotion programmes executed during the same time period, which experienced similar recruitment problems. The low response rate might have biased the results. The worksites that participated are most likely not representative of the average worksite, in that the enrolled worksites probably showed a higher interest in health promotion than worksites in general (Sorensen, et al., 1996). Implementing the project among less interested worksites might not have generated the same results. Secondly, the participation rate of individual participants per worksite was low, which may have resulted in a selective population. An indication that selection bias may have occurred is the overrepresentation of participants who were characterized as motivated to change. This corresponds with the findings of other worksite health promotion programmes (Linnan et al., 2001). Furthermore, a relatively high response of older and overweight individuals was observed, even though the aim of the project was to prevent weight gain in young adults. This is also in line with observations in other studies (Rimm et al., 1995; Baik et al., 2000) and further supports the presence of a selected population. It is unsure whether the same results would have been observed in younger, leaner or less motivated individuals.

Not withstanding the low participation rates among both worksites and individual participants an important strength of the project was conducting the programme in a so-called 'real-life' setting, thus providing information on the potential effectiveness of the programme in daily health promotion practice.

\section{Study design}

The study conducted in this thesis employed a quasi-experimental pre-test multiple post-test control group design to test the effectiveness of the programme. However, in order to test the effectiveness of the intervention stimulating stair use by the formation of implementation intentions a randomized control trail was employed. As was pointed out in Chapter 2 and earlier in this discussion section the original evaluation design included randomization. However, as a result of recruitment pro- 
blems the design was replaced by a quasi-experimental design. What distinguishes a quasi-experimental design from a RCT is that in the former units are not assigned to conditions randomly as done in a RCT. The RCT is therefore often referred to as the gold standard, especially in a research area as medicine. It is believed that the observed outcome differences between groups are likely due to the treatment and not to differences between the groups as they are assumed to be similar to each other as a result of randomization. In a quasi-experimental design on the other hand it is often assumed that groups may differ in many systematic ways other than due to the treatment, which could lead to alternative explanations for the observed effect (Shadish et al. 2002). In order to minimize alternative explanations of the observed effects we applied two approaches. Firstly, worksites were matched according to the socio-economic status of their employees before assigning them to the intervention and control group, this in order to ensure the comparability between the groups. Secondly, data on several individual and worksite characteristics, as well as outcome measures were collected to gain insight into differences between groups in their initial values. If so, allowances were made for these differences in the analyses. Even though the applied alternative to the randomized control trial (RCT) has been commonly advocated by health promotion researchers (Pelletier, 2001), and two approaches were applied to minimize alternative explanations of the observed effects, dropping the RCT design may have lowered the validity of the observed results. Without randomization is it very difficult to estimate the causal effect of the intervention, thus to conclude if the observed differences between the groups were really due to the intervention.

\section{Measurement of WGPBs, motivation to change and corresponding cognitions}

Several issues regarding the assessment of WGPBs, motivation to change and their corresponding cognitions should be addressed. First some overall concerns regarding self-reports will be discussed, additionally concerns related to the specific measures will be addressed separately. The first measurement issue relates to the use of self-reports for the assessment of changes in WGPBs, motivation to change and corresponding cognitions. In general self-reported data are prone to measurement error due to challenges of memory, estimation and bias (NICE clinical guideline, 2006). In addition social desirability, the tendency of individuals in keeping with perceived societal norms and beliefs (Edwards, 1957; Edwards, 1961), and social approval, the need to obtain a positive response in a testing situation (Larsen et al., 1976), may also lead to measurement error.

The second measurement issue is related to the choice of appropriate methods for measuring the WGPBs. There is no 'gold standard' for measuring dietary intake and its change over time. However, when a dietary intervention trial involves a large number of subjects who will be followed longitudinally with multiple measurement time points, the food frequency questionnaire (FFQ) is the most commonly used and cost-effective dietary measurement method (Thomson et al., 2003). In the study conducted in this thesis a FFQ was used for the measurement of the dietary intake related WGPBs. The FFQ was partly validated among the Dutch adult population (Camps \& Abden, 1994). There are some general concerns regarding measurement errors associated with FFQs. The first concerns the lack of detail and specificity of the foods listed, as FFQs are largely composed of frequentlyconsumed foods(NIH-guide, 2002). For thepresentstudyaFFQwaschosen that corresponded as much as possible with the objective of measuring changes in the dietary intake related WGPBs. An additional concern is the intake-related bias, for example over reporting of supposedly healthy foods and underreporting of supposedly unhealthy foods (Kipnis et al., 1999). Moreover, the level of under- and over reporting 
may vary in subjects with different body mass (Goris et al, 2000). The fact that the FFQ was short, simple and corresponded with our objective to measure changes in the dietary intake related WGPBs outweighed these proposed limitations of the FFQ.

In contrast to measuring dietary intake and its changes over time a 'golden' standard has been proposed for the measurement of physical activity, namely the accelerometer, which has been suggested to provide the most objective and detailed record of physical activity (Welk et al., 2000). Although such activity monitors offer considerable promise for providing valid assessments of physical activity, their application in large-scale studies is constrained by cost and technical complexity (Livingstone et al., 2003). A less costly and more practical measure of physical activity, especially among larger-scale studies, is a self-report measure. A frequency questionnaire was therefore applied for measuring changes in the physical activity related WGPBs. An overall concern of physical activity questionnaires is that many aspects of physical activity, especially those that are associated with low to moderate intensity, are routine and therefore not salient enough to be recalled well by a respondent (NICE clinical guideline, 2006). Additionally, there are some limitations specifically related to the questionnaire used in the present thesis. As mentioned in Chapter 3 and in an earlier section of this discussion our results showed that the usefulness of the questionnaire in estimating physical activity levels as activity scores, which combine time spent in physical activities with an estimation of intensity and therefore can be used as measure of total physical activity-associated energy expenditure, was smaller than expected from a previous study which had validated the questionnaire in another sample of the adult working Dutch population (Wendel-Vos et al., 2001). More reliable estimations were obtained with the questionnaire when total time spent on physical activities was used. In order to measure changes in the physical activity related WGPBs we therefore chose to measure changes in reported minutes instead of changes in activity scores, thus ignoring subjective estimations of intensity in the questionnaire. Although the questionnaire might be limited in estimating physical activity levels we do belief that it has practical value in monitoring changes in individual physical activity.

The final measurement issue relates to the operationalization of the measures related to the cognitive factors of the WGPBs. For the study conducted in the present thesis a choice was made to operationalize them with 'watching one's weight' as the behavioural object. Individuals might however not associate the specified WGPBs with watching their weight. So when participants were asked to indicate their attitude towards watching their weight, this might have resulted in a different score than the sum of items specifically related to the various WGPBs. This has to be taken into consideration when interpretating the results related to the cognitive factors of the WGPBs.

Notwithstanding the above-mentioned shortcomings of the applied selfreport measures, the present combination of collecting both so called 'hard' and 'soft' outcome measures, over a period of two years has made a unique contribution to the effect evaluation. Only few studies in health promotion have applied such a diverse set of theoretically and empirically based outcome indicators.

\section{Process evaluation}

Response to the items regarding the process evaluation was very low, making these data inappropriate for in-depth analyses of effective intervention components or dose-response examinations. The low response could either be a result of the length of the questionnaire, as these questions were placed at the end, or this could indicate that participants were dissatisfied with the intervention. The data that were 
collected indicated that more than half of the participants found the intervention materials to be interesting and comprehensible, especially the personal feedback of body composition measures. The computer-tailoring CD-Rom regarding Energy Balance was least appreciated.

Observations and structured interviews with intermediaries revealed that only two out of six worksites formed a linkage board. The rationale for forming a linkage board within each worksite was that individuals within this board would be able to facilitate collaboration or influence changes necessary to support the adoption, implementation, and institutionalization of the environmental components of the programme. The linkage board was therefore responsible for the adoption and implementation of the worksite-based interventions, guided by the provided handbook they were assisted through the different stages of diffusion and had the opportunity to select interventions that match the needs and possibilities of their worksite. In the worksites that did not form a linkage board, the contact person or someone assigned by the contact person was responsible for the implementation. Despite the less successful implementation of the worksite linkage-boards, four worksites did implement multiple environmental interventions, which varied from articles in the personnel magazine to offering workshops on healthy dietary behaviour given by a dietician. The intervention directed at stimulating stair use was implemented in all these four worksites.

\section{IMPLICATIONS OF THE FINDINGS}

The results presented in this thesis have several implications for practice and future research. Recommendations for practice are related to the implementation and dissemination of the NHF-NRG In Balance-project or other worksite-based weight gain prevention interventions. Implications for future research are based on questions that have remained unanswered during the studies conducted in this thesis.

\section{Recommendations for practice}

The NHF-NRG In Balance-project was found to be effective with regard to changes in the sum of skinfolds and waist circumference and resulted in small to medium and statistically significant effects on several of the promoted weight gain preventive behaviours both at 12 and 24 months. But before a decision can be made about the programmes readiness to be implemented on a larger scale there are some practical implications that need to be taken into consideration. Even though worksites present extensive opportunities to reach a large number of people and provide many opportunities to reinforce the adoption and maintenance of healthy lifestyle behaviours there are substantial barriers that need to be overcome. Firstly, worksites need to be motivated to participate in health promoting programmes. This could be done by implementing a short intervention directed primarily at motivating worksites prior to the implementation of the present programme. The emphasis should be on highlighting the benefits of participation for the individual worksite, i.e. improving worker morale, decreasing absenteeism, and improving behaviours associated with increased worker productivity (US Department of Health and Human Services, 1993) and on collaboration, this in order to ensure more empowerment and adjustments to the needs of the worksites. Additionally, a national concept could be created on the basis of which worksites can distinguish themselves as being a health promoting worksite, when participating in programmes such as the one presented in this thesis. This could be done in collaboration with the Workplace Health Promotion, NIGZ (the Netherlands Institute for Health Promotion and Disease Prevention) Work \& 
Health, which has developed such tool, named the certificate 'Model of Good Practice' (Gezondheidsmanagement, 2007). The Workplace Health Promotion cluster within this institute is concerned with activities that promote health in the workplace.

The second practical implication is related to motivating individuals to participate in a worksite based weight gain prevention programme, specifically young adults with a healthy weight. Prior to intervention dissemination, more emphasis should be placed on increasing the awareness of these individuals with regard to their risk of gaining weight. This could be done by organizing a worksite-broad 'kick-off' before to the start of the intervention, which is purely focused on triggering individuals to participate. In addition to the individual characteristic, previous worksite intervention programmes have proposed several worksite characteristics as predictors of participation, such as social norm, work-pace, participatory strategies and management style (Linnan et al., 2001). Issues regarding these characteristics could be addressed in the proposed pre-intervention programme, such as stimulating the creation of an organizational climate, in which the management is supportive of participation. This could also have a beneficial effect on the formation of the worksite linkage boards.

The third practical implication is related to recommendations concerning the intervention content. As weight gain is a result of an imbalance between energy intake and energy expended, interventions should focus on both behaviours. Interventions should promote behaviours that fit easily into people's everyday life, that are tailored to people's individual preferences and circumstances and that are aimed at improving people's belief in their ability to change. Moreover, a comprehensive approach should be applied over a long-term period, which includes both motivational and environmental interventions (NICE-report, 2006).

Fourthly, most intervention components of NHF-NRG In Balance can be implemented at a larger scale without practical concerns. There is, however, a practical concern regarding the implementation of the well-appreciated intervention component that provided feedback on body composition measures on a larger scale. In the current study measurements were performed by the research team, who accordingly composed the personal feedback. Implementing this on a broader scale could either include self-weighing, in combination with a computer tailoring programme in order to distribute the personal feedback or the involvement of company doctors, who could regularly execute the measurements. The second individual intervention that involves a practical implication is the intervention component promoting stair use by the formation of implementation intentions, as the formation was stimulated by the research team during the execution of the anthropometric measurements. Stimulating the formation of implementation intentions on a larger scale could be executed by sending employees an e-mail containing the procedure of forming an implementation intention.

\section{Suggestions for future research}

The different studies that have been conducted in this thesis have shed light on issues regarding the effectiveness of the NHF-NRG In Balance-project. Consequently, several recommendations for future research can be made. As discussed in the methodological section of this discussion recruitment of both worksites as individual participants proved difficult. In order to optimize participation efforts should be explored regarding the re-examination of recruitment strategies. Only when high participation rates are observed, especially among individual participants, may the full public health impact be realized.

Another avenue for future research relates to the development and validation of a self-report measure for assessing changes in physical activity specifically 
aimed at the worksite. This should include more specific questions on occupational physical activity, in order to fully understand changes in the amount of physical activity performed at the worksite. Additionally, more insight into work-related physical activity is needed, especially in relation to total physical activity, as it has been suggested that individuals who have physically active jobs are less active during leisure time (Schneider \& Becker, 2005). However, no accurate tools have been used to measure occupational physical activity.

Even though the overall aim of this thesis was to test the effectiveness of the developed programme as a whole, it would be interesting to further explore possible effective components. Analyses could be conducted on the sub-population of individuals who were indeed able to prevent weight gain, or on a sub-population of individuals who fully participated in the programme and used the intervention components as suggested.

Furthermore, in correspondence with the CONSORT statement (Moher et al., 2001), we advocate that studies should report participation rates among approached worksites in publications on worksite intervention effects as well as indicating expected participation rates in research proposals. Health promotion journals should consider implementing similar guidelines regarding in-depth reporting of the research process, giving readers the possibility to appropriately evaluate its validity. Such information will help to draw conclusions on the practical relevance of the shown effectiveness of the intervention, and thus, the evidence-base in health promotion will be optimised.

The final avenue for future research relates to the environmental interventions and more specifically to the mediated or unmediated influence of these interventions on the observed behaviour change. Health education research has mostly focused on the mediated environmental influences, in which environments are hypothesized to impact behaviours through their influence on cognitive factors (Crosby et al., 2002). However, empirical evidence is growing with regard to the importance of largely unmediated environmental influences on energy balance-related behaviours (Kremers et al., 2006). Rather than just focusing on the effectiveness of the programme, future research should pay more attention to how the programme has had its impact on behaviour. 
REFERENCES 
- Aadahl, M., \& Jorgensen, T. (2003). Validation of a new self-report instrument for measuring physical activity. Medicine and Science in Sports and Exercise, 35, 1196-1202.

- Aarts, H., \& Dijksterhuis, A. (2000a). The automatic activation of goal-directed behaviour: the case of travel habit. Journal of Environmental Psychology, 20, 75-82.

- Aarts, H., \& Dijksterhuis, A. (2000b). Habits as knowledge structures: automaticity in goal-directed behaviour. Journal of Personality and Social Psychology, 78, 53-63.

- Adams, J.F., \& Engstrom, A. (2000). Helping consumers achieve recommended intakes of whole grain foods. Journal of the American College of Nutrition, 19, 339S-344S.

- Ainslie, P.N., Reilly, T., \& Westerterp, K.R. (2003). Estimating human energy expenditure: a review of techniques with particular reference to doubly labelled water. Sports Medicine, 33, 683-698.

- Ainsworth, B.E., Haskell, W.L., Leon, A.S., Jacobs, D.R. Jr., Montoye, H.J., Sallis, J.F., \& Paffenbarger, R.S. (1993). Compendium of physical activities: classification of energy costs of human physical activities. Medicine and Science in Sports and Exercise, 25, 71-80.

- Ainsworth, B.E., Sternfeld, B., Richardson, M.T., \& Jackson, K. (2000). Evaluation of the kaiser physical activity survey in women. Medicine and Science in Sports and Exercise, 32, 1327-1338.

- Ajzen, I. (1991). The Theory of Planned Behaviour. Organizational Behaviour and Human Decision Processes, 50, 179-211.

- Aldana, S.G., \& Pronk, N.P. (2001). Health promotion programs, modifiable health risks, and employee absenteeism. Journal of Occupational Environmental Medicine, 43, 36-46.

- Andersen, R.E., Franckowiak, S.C., Snyder, J., Bartlett, S.J., \& Fontaine, K.R. (1998). Can inexpensive signs encourage the use of stairs? Results from a community intervention. Annals of Internal Medicine, 129, 363-369.

- Andersen, R.E., Franckowiak, S.C., Zuzak, K.B., Cummings, E.S.,. \& Crespo, C.J. (2000). Community interventions to encourage stair use among African-American commuters. Medicine and Science in Sports and Exercise, 32, S38.

- Astrup, A., Grunwald, GK., Melanson, E.L., Saris, W.H., \& Hill, J.O. (2000a). The role of low-fat diets in body weight control: a meta-analysis of ad libitum dietary intervention studies. International Journal of Obesity, 24, 1545-1552.

- Astrup, A., Ryan, L., Grunwald, G.K., Storgaard, M., Saris, W., Melanson, E., \& Hill J.O. (2000b). The role of dietary fat in body fatness: evidence from a preliminary metaanalysis of ad libitum low-fat dietary intervention studies. British Journal of Nutrition, 83, S25-S32.

- Astrup, A., Buemann, B., Flint, A., \& Raben, A. (2002). Low-fat diets and energy balance: how does the evidence stand in 2002? The Proceedings of the Nutrition Society, 61, 299-309.

- Bagwell, M.M., \& Bush, H.A. (2000). Improving health promotion for blue-collar workers. Journal of Nursing Care Quality, 14, 65-71.

- Baik, I., Ascherio, A., Rimm, E.B., Giovannucci, E., Spiegelman, D., Stampfer, M.J., \& Willett, W.C. (2000). Adiposity and mortality in men. American Journal of Epidemiology, 152, 264-271.

- Ball, K., Bauman, A., Leslie, E., \& Owen, N. (2001). Perceived environmental aesthetics and convenience and company are associated with walking for exercise among Australian adults. Preventive Medicine, 33, 434-440.

- Bandura, A. (1986). Social foundations of thought and action: A social cognitive theory. Englewood Cliffs: Prentice-Hall.

- Bandini, L.G., Schoeller, D.A., Cyr, H.N., \& Dietz, W.H. (1990). Validity of reported energy intake in obese and non-obese adolescents. American Journal of Clinical Nutrition, 52, 421-425. 
- Baranowski, T., Cullen, K.W., \& Baranowski, J. (1999). Psychosocial correlates of dietary intake: advancing dietary intervention. Annual Review of Nutrition, 19, 17-40.

- Bargh, J.A., \& Gollwizter, P.M. (1994). Environmental control of goal-directed action: Automatic and strategic contingencies between simulations and behavior. Nebraska Symposium on Motivation, 41, 71-124.

- Bartholomew, L.K., Parcel, G.S., Kok, G., \& Gottlieb, N.H. (2001). Intervention Mapping: Designing theory-and evidence-based health promotion programs. Mountain View: Mayfield.

- Bassett, D.R. Jr., Ainsworth, B.E., Swartz, A.M., Strath, S.J., O’Brien, W.L., \& King, G.A. (2000). Validity of four motion sensors in measuring moderate intensity physical activity. Medicine and Science in Sports and Exercise, 32, S471-S480.

- Bennet, S. (1996). Socioeconomic inequalities in coronary heart disease and stroke mortality among Australian men, 1979-1993. International Journal of Epidemiology, 25, 266-275.

- Beresford, S.A., Thompson, B., Feng, Z., Christianson, A., McLerran, D., \& Patrick, D.L. (2001). Seattle 5 a Day worksite program to increase fruit and vegetable consumption. Preventive Medicine, 32, 230-238.

- Biener, L., Glanz, K., McLerran, D., Sorensen, G., Thompson, B., Basen-Engquist, K., Linnan, L., \& Varnes, J. (1999). Impact of the Working Well Trial on the worksite smoking and nutrition environment. Health Education \& Behaviour, 26, 478-494.

- Blamey, A., Mutrie, N., \& Aitchison, T. (1995). Health promotion by encouraged use of stairs. British Medical Journal, 311, 289-290.

- Boekaerts, M., Rintrich, P.R., \& Zeider, M. (2000). Handbook of self-regulation. San Diego, CA: Academic Press.

- Boreham, C.A.G., Wallace, W.F.M., \& Nevill, A. (2000). Training effects of accumulated daily stair-climbing exercise in previously sedentary young women. Preventive Medicine, 30, 277-281.

- Boreham, C.A.G., Kennedy, R.A., Murphy, M.H., Tully M., Wallace, W.F., \& Young, I. (2005). Training effects of short bouts of stair climbing on cardiorespiratory fitness, blood lipids, and homocysteine in sedentary young women. British Journal of Sports Medicine, 39, 590-593.

- Boutelle, K.N., Jeffery, R.W., Murray, D.M., \& Schmitz, M.K. (2001). Using signs, artwork, and music to promote stair use in a public building. American Journal of Public Health, 91, 2004-2006.

- Brandstatter, V., Lengfelder, A., \& Gollwitzer, P.M. (2001). Implementation intentions and efficient action initiation. Journal of Personality and Social Psychology, 81, 946-960.

- Brenner, H., \& Kliebsch, U. (1996). Dependence of weighted kappa coefficients on the number of categories. Epidemiology, 7, 199-202.

- Brodie, D., Moscrip, V., \& Hutcheon, R. (1998). Body composition measurement: a review of hydrodensitometry, anthropometry, and impedance methods. Nutrition, 14, 296-310.

- Brown, W.J., \& Trost, S.G. (2003). Life transitions and changing physical activity patterns in young women. American Journal of Preventive Medicine, 25, 140-143.

- Brownell, K.D., Stunkard, A.J., \& Albaum, J.M. (1980). Evaluation and modification of exercise patterns in the natural environment. American Journal of Psychiatry, 137, 1540-1545.

- Brownson, R.C., Boehmer, T.K., \& Luke, D.A. (2005). Declining rates of physical activity in the United States: What are the contributors? Annual Review of Public Health, 26, 421-443.

- Brug, J., Debie, S., Van Assema, P., \& Weijts, W. (1995). Psychosocial determinants of fruit and vegetable consumption among adults: results of focus group interviews. Food Quality and Preference, 6, 99-107. 
- Brug, J., Glanz, K., \& Kok, G. (1997). The relationship between self-efficacy, attitudes, intake compared to others, consumption, and stages of change related to fruit and vegetables. American Journal of Health Promotion, 12, 25-30.

- Brug, J., Campbell, M., \& Van Assema, P. (1999). The application and impact of computer-generated personalized nutrition education: a review of the literature. Patient Education and Counselling, 36, 145-156.

- Brug, J., Van Lenthe, F., \& Kremers, S.P.J. (2006). Revisiting Kurt Lewin: How to gain better insight in environmental determinants of obesogenic behaviours? American Journal of Preventive Medicine, 32, 163-172.

- Bull, S.S., Gillette, C., Glasgow, R.E., \& Estabrooks, P. (2003). Work site health promotion research: to what extent can we generalize the results and what is needed to translate research to practice? Health Education \& Behaviour, 30, 537-549.

- Burke, G.L., Bild, D.E., Hilner, J.E., Folsom, A.R., Wagenknecht, L.E., \& Sidney, S. (1996). Differences in weight gain in relation to race, gender, age and education in young adults: the CARDIA Study. Coronary Artery Risk Development in Young Adults. Ethnical Health, 1, 327-335.

- Burton, N.W., \& Turrell, G. (2000). Occupation, hours worked, and leisure-time physical activity. Preventive Medicine, 31, 673-681.

- Calfas, K.J., Sallis, J.F., Zabinski, M.F., Wilfley, D.E., Rupp, J., Prochaska, J.J., Thompson, S., Pratt, M., \& Patrick, K. (2002). Preliminary evaluation of a multicomponent program for nutrition and physical activity change in primary care: $\mathrm{PACE}+$ for adults. Preventive Medicine, 34, 153-161.

- Campbell, M.K., Tessaro, I., DeVellis, B., Benedict, S., Kelsey, K., Belton, L., \& HenriquezRoldan, C. (2000). Tailoring and targeting a worksite health promotion program to address multiple health behaviours among blue-collar women. American Journal of Health Promotion, 14, 306-313.

- Camps, M., \& Abden, D. (1994). De ENVET-lijst: Ontwikkeling en validering van een korte voedelsfrequentievragenlijst naar energie en vet- tweede fase. [The ENVET-list: Development and validation of a short food frequency questionnaire for energy and fat- second phase]. Geldrop-Valkenswaard: GGD regio Geldrop-Valkenswaard.

- Carraro, R., \& Garcia Cebrian, M. (2003). Role of prevention in the contention of the obesity epidemic. European Journal of Clinical Nutrition, 57, S94-S96.

- Caterson, I.D., \& Gill, T.P. (2002). Obesity: epidemiology and possible prevention. Best Practice and Research. Clinical Endocrinology Metabolism, 16, 595-610.

- Chan, J.M., Rimm, E.B., Colditz, G.A., Stampfer, M.J., \& Willet, W.C. (1994). Obesity, fat distribution, and weight gain risk as risk factors for clinical diabetes in men. Diabetes Care, 17, 961-969.

- Chasan-Taber, L., Erickson, J.B., Nasca, P.C., Chasan-Taber, S., \& Freedson, P.S. (2002). Validity and reproducibility of a physical activity questionnaire in women. Medicine and Science in Sports and Exercise, 34, 987-992.

- Chen, R., \& Tunstall-Pedoe, H. (2005). Socioeconomic deprivation and waist circumference in men and women: the Scottish MONICA surveys 1989-1995. European Journal of Epidemiology, 20, 141-147.

- Cohen, J. (1988). Statistical power analysis for the behavioural sciences. (2nd ed.) Hillsdale, NJ, Lawrence Earlbaum Associate.

- Coleman, K.J., \& Gonzalez, E.C. (2001). Promoting stair use in a US-Mexico border community. American Journal of Public Health, 91, 2007-2009.

- Computer Science and Applications Inc. Activity monitor operator's manual; model 7164; version AM7164-2.2. (1999). Shalimar (FL): Computer Science and Applications, Inc, Ambulatory Monitoring Applications Division. 
- Craig, C.L., Brownson, R.C., Cragg, S.E., \& Dunn, A.L. (2002). Exploring the effect of the environment on physical activity: a study examining walking to work. American Journal of Preventive Medicine, 23, 36-43.

- Crespo, C.J., Ainsworth, B.E., Keteyina, S.J., Heath, G.W., \& Smith, E. (1999). Prevalence of physical inactivity and its relation to social class in U.S. adults: results from the Third National Health and Nutrition Examination Survey, 1988-1994. Medicine and Science in Sports and Exercise, 31, 1821-1827.

- Crosby, R.A., Kegler, M.C., \& DiClemente, R.J. (2002). Understanding and applying theory in health promotion practice and research. In R.J. DiClemente, R.A.Crosby, M.C. Kegler (Eds.). Emerging theories in Health Promotion Practice and Research: Strategies for improving the Public Health. San Francisco, CA: Josey-Bass.

- Davey, R.C. (2004). The obesity epidemic: too much food for thought? British Journal of Sports Medicine, 38, 360-363, discussion 363.

- De Bruijn, G.J., Kremers, S.P.J., Schaalma, H., Van Mechelen, W., \& Brug, J. (2005). Determinants of adolescent bicycle use for transportation and snacking behaviour. Preventive Medicine, 40, 658-667.

- De Ridder, D.T.D., \& De Wit, J.B.F.(2006). Self-regulation in Health Behaviour: concepts, theories, and central issues. In: D.T.D. De Ridder \& J.B.F. De Wit (Eds.), Self-regulation in Health Behaviour: John Wiley \& Sons, Ltd.

- Dholakia, U.M., \& Bagozzi, R.P. (2003). As time goes by: How goal and implementation intentions influence enactment of short-fuse behaviours. Journal of Applied Social Psychology, 33, 889-922.

- Dishman, R.K., Oldenburg, B., O’Neal, H., \& Shephard, R.J. (1998). Worksite physical activity interventions. American Journal of Preventive Medicine, 15, 344-361.

- Douketis, J.D., Feightner, J.W., Attia, J., \& Feldman, W.F. (1999). Periodic health examination, 1999 update, 1. Detection, prevention and treatment of obesity. Canadian Task Force on Preventive Health Care, 160, 513-525.

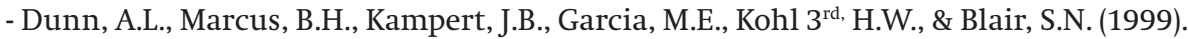
Comparison of lifestyle and structured interventions to increase physical activity and cardiorespiratory fitness: a randomized trial. JAMA, 281, 327-334.

- Durante, R., \& Ainsworth, B.E. (1996). The recall of physical activity: using a cognitive model of the question-answering process. Medicine and Science in Sports and Exercise, 28, 1282-1291.

- Edwards, A.L. (1957). Social desirability and probability or endorsement of items in the interpersonal check list. Journal of Abnormal Psychology, 55, 394-396.

- Edwards, A.L. (1961). Social desirability or acquiescence in the MMPI? A case study with the SD scale. Journal of Abnormal Psychology, 63, 351-359.

- Egger, G., \& Swinburn, B. (1997). An "ecological” approach to the obesity pandemic. British Medical Journal, 315, 477-480.

- Eiben, G., \& Lissner, L. (2006). Health Hunters-an intervention to prevent overweight and obesity in young high-risk women. International Journal of Obesity, 30, 691-696.

- Ello-Martin, J.A., Ledikwe, J.H., \& Rolls, B.J. (2005). The influence of food portion size and energy density on energy intake: implications for weight management. American Journal of Clinical Nutrition, 82, S236-S241.

- Emmons, K.M., Thompson, B., McLerran, D., Sorensen, G., Linnan, L., Basen-Enquist, K., \& Biener, L. (2000). The relationship between organizational characteristics and the adoption of workplace smoking policies. Health Education $\&$ Behaviour, 27(4), 483-501.

- Engbers, L.H., van Poppel, M.N., Chin, A.P.M.J., \& van Mechelen, W. (2005). Worksite health promotion programs with environmental changes: a systematic review. American Journal of Preventive Medicine, 29, 61-70. 
- Eriksen, K., Haraldsdottir, J., Pederson, R., \& Flyger, H.V. (2003). Effect of a fruit and vegetable subscription in Danish schools. Public Health Nutrition, 6, 57-63.

- Eves, F.F., \& Webb, O.J. (2006a). Worksite interventions to increase stair climbing, reasons for caution. Preventive Medicine, 43, 4-7.

- Eves, F.F., Webb, O.J., \& Mutrie, N. (2006b). A workplace intervention to promote stair climbing: greater effects in the overweight. Obesity, 14, 2210-2216.

- Field, A. (2003). Discovering Statistics Using SPSS for Windows. London: SAGE Publications Ltd.

- Flegal, K.M., Carroll, M.D., Ogden, C.L., \& Johnson, C.L. (2002). Prevalence and trends in obesity among US adults, 1999-2000. JAMA, 288, 1723-1727.

- Fogelholm, M., Hiilloskorpi, H., Laukkanen, R., Oja, P., van Marken Lichtenbelt, W., \& Westerterp, K. (1998). Assessment of energy expenditure in overweight women. Medicine and Science in Sports and Exercise, 30, 1191-1197.

- Forster, J.L., Jeffery, R.W., Schmid, T.L., \& Kramer, F.M. (1988). Preventing weight gain in adults: a pound of prevention. Health Psychology, 7, 515-525.

- Foster, C., \& Hillsdon, M. (2004). Changing the environment to promote healthenhancing physical activity. Journal of Sports Sciences, 22, 755-769.

- French, S.A, Story, M., \& Jeffery, R.W. (2001). Environmental influences on eating and physical activity. Annual Review of Public Health, 22, 309-335.

- Galobardes, B., Morabia, A., \& Bernstein, M.S. (2000). The differential effect of education and occupation on body mass and overweight in a sample of working people of the general population. Annals of Epidemiology, 10, 532-537.

- Gateway to the European Union. Available at: http://europa.eu.int. Accessed July 14, 2005.

- Gezondheidsmanagement. Avaiable at: http://www.gezondheidsmanagement.nl/ Accessed April 23, 2007.

- Giles-Corti, B., \& Donovan, R.J. (2003). Relative influences of individual, social environmental, and physical environmental correlates of walking. American Journal of Public Health, 93, 1583-1589.

- Glanz, K., Sorensen, G., \& Farmer, A. (1996). The health impact of worksite nutrition and cholesterol intervention programs. American Journal of Health Promotion, 10, 453470.

- Glanz, K., Sallis, J.F., Saelens, B.E., \& Frank, L.D. (2005). Healthy nutrition environments: concepts and measures. American Journal of Health Promotion, 19, 330-333.

- Glasgow, R.E., Terborg, J.R., Hollis, J.F., Severson, H.H., \& Boles, S.M. (1995). Take heart: results from the initial phase of a work-site wellness program. American Journal of Public Health, 85, 209-216.

- Glasgow, R.E., Terborg, J.R., Strycker, L.A., Boles, S.M., \& Hollis, J.F. (1997). Take Heart II: replication of a worksite health promotion trial. Journal of Behavioural Medicine, 20, 143-161.

- Glenny, A.M., O’Meara, S., Melville, A., Sheldon, T.A., \& Wilson C. (1997). The treatment and prevention of obesity: a systematic review of the literature. International Journal of Obesity, 21, 715-737.

- Gollwitzer, P.M. (1990). Action phases and mind-sets. In E.T. Higgins, \& R.M. Sorrentino (Eds.). Handbook of motivation and cognition: Foundations of social behaviour. New York: Guilford.

- Gollwitzer, P.M. (1993). Goal achievement: The role of intentions. European Review of Social Psychology, 4, 141-185.

- Gollwitzer, P.M. (1996). The volitional benefits of planning. In P.M. Gollwitzer \& J.A. Bargh (Eds.). The psychology of action: Linking cognition and motivation to behavior (pp. 287-312). New York: Guilford Press. 
- Gollwitzer, P.M., \& Oettingen, G. (1998). The emergence and implementation of health goals. Psychology and Health, 13, 687-715.

- Gollwitzer, P.M. (1999). Implementation Intentions: strong effects of simple plans. American Psychologist, 54, 493-503.

- Gollwitzer, P.M., \& Sheeran, P. (2006). Implementation intentions and goal achievement: a meta-analysis of effects and processes. Advances in Experimental Social Psychology, 38, 69-119.

- Goodman, R.M., Stackler, A., \& Kegler, M.C. (1997). Mobilizing organizations for health enhancement: theories of organizational change. In: K. Glanz, F.M. Lewis, B.K. Rimer (Eds.). Health behaviour and health education: theory, research and practice (pp. 287-312). San Fransisco: Jossey-Bass.

- Goris, A.H., Westerterp-Plantenga, M.S., \& Westerterp, K.R. (2000). Undereating and underrecording of habitual food intake in obese men: selective underrecording of fat intake. American Journal of Clinical Nutrition, 71, 130-134.

- Hagdrup, N.A., Simoes, E.J., \& Brownson, R.C. (1998). Fruit and vegetable consumption in Missouri: knowledge, barriers and benefits. American Journal of Health Behaviour, 22, 90-100.

- Hammond, S.L., Leonard, B., \& Fridinger, F. (2000). The Centers for Disease Control and Prevention Director's Physical Activity Challenge: an evaluation of a worksite health promotion intervention. American Journal of Health Promotion, 15, 17-20.

- Hardeman, W., Griffin, S., Johnston, M., Kinmonth, A.L., \& Wareham, N.J. (2000). Interventions to prevent weight gain: a systematic review of psychological models and behaviour change methods. International Journal of Obesity, 24, 131-143.

- Harting, J., van Assema, P., Ruland, E., van Limp, P., Gorgels T., van Ree, J, Vermeer, F., \& de Vries, N.K. (2005). Implementation of an innovative health service a real-world diffusion study. American Journal of Preventive Medicine, 29, 113-119.

- Heaney, C.A., \& Israel, A. (1997). Social networks and social support. In K. Glanz, F.M. Lewis, B.K. Rimer (Eds.). Health behaviour and health education: Theory, research and practice. (pp. 179-205). San Francisco: Jossey-Bass.

- Hendelman, D., Miller, K., Baggett, C., Debold, E., \& Freedson, P. (2000). Validity of accelerometry for the assessment of moderate intensity physical activity in the field. Medicine and Science in Sports and Exercise, 32, S442-S449.

- Hennrikus, D.J., \& Jeffery, R.W. (1996). Worksite intervention for weight control: a review of the literature. American Journal of Health Promotion, 10, 471-498.

- Hill, J.O., \& Peters, J.C. (1998). Environmental contributions to the obesity epidemic. Science, 280, 1371-1374.

- Hill, J.O., Wyatt, H.R., Reed, G.W., \& Peters, J.C. (2003). Obesity and the environment: where do we go from here? Science, 299, 853-855.

- Hill, J.O. (2006). Understanding and addressing the epidemic of obesity: an energy balance perspective. Endocrine Reviews, 27, 750-761.

- Hillsdon, M., Foster, C., \& Thorogood, M. (2005). Interventions for promoting physical activity, Cochrane Database Systematic Reviews, 25, CD003180.

- Hitchcock, P.N., \& Pugh, J.A. (2002). A clinical review: Management of overweight and obese adults. British Medical Journal, 325, 757-761.

- Howarth, N.C., Saltzman, E., \& Roberts, S.B.(2001). Dietary fibre and weight regulation. Nutrition Reviews, 59, 129-139.

- Howarth, N.C., Huang, T.T., Roberts, S.B., \& McCrory, M.A. (2005). Dietary fibre and fat are associated with excess weight in young and middle-aged US adults. Journal of the American Dietetic Association, 105, 1365-1372.

- Jackson, C., Lawton, R., Knapp, P., Raynor, D.K., Conner, M., Lowe, C., \& Closs, S.J. (2005). Beyond intention: Do specific plans increase health behaviors in patients in 
primary care? A study of fruit and vegetable consumption. Social Science and Medicine, 60, 2383-2391.

- Jager, W., Boers, S., Eckringa, C., \& Westerhof, J.D. (1996). The efficacy of prompting in changing habitual behavior. Nederlands tijdschrift voor de psychologie, 51, 173-182.

- Jakicic, J.M. (2002). The role of physical activity in prevention and treatment of body weight gain in adults. Journal of Nutrition, 132, S3826-S3829.

- Jakicic, J.M., \& Otto, A.D. (2005). Physical activity considerations for the treatment and prevention of obesity. American Journal of Clinical Nutrition, 82, S226-S229.

- James, A.S., Campbell, M.K., \& Hudson, M.A. (2002). Perceived barriers and benefits to colon cancer screening among African Americans in North Carolina: how does perception relate to screening behaviour? Cancer Epidemiology, Biomarkers and Prevention, 11, 529-534.

- Jeffery, R.W., \& French, S.A. (1999). Preventing weight gain in adults: the pound of prevention study. American Journal of Public Health, 89, 747-751.

- Jeffery, R.W. (2001). Public health strategies for obesity treatment and prevention. American Journal of Health Behaviour, 25, 252-259.

- Jurg, M.E., Kremers, S.P.J., Candel, M.J.J.M., van der Wal, M.F., \& de Meij, J.S.B. (2006). A controlled trial of a school-based environmental intervention to improve physical activity in Dutch children: JUMP-in, kids in motion. Health Promotion International, 21, 320-330.

- Kahn, E.B., Ramsey, L.T., Brownson, R.C., Heath, G.W., Howze, E.H., Powell, K.E., Stone, E.J., Rajab, M.W., \& Corso, P. (2002). The effectiveness of interventions to increase physical activity. A systematic review. American Journal of Preventive Medicine, 22, 73-107.

- Katz, D.L., O’Connell, M., Yeh, M.C., Nawaz, H., Njike, V., Anderson, L.M., Cory, S., Dietz, W. (2005). Public health strategies for preventing and controlling overweight and obesity in school and worksite settings: a report on recommendations of the Task Force on Community Preventive Services. MMWR. Recommendations and Reports: Centers for Disease Control, 54, 1-12.

- Kemper, H.C.G., Stasse-Wolthuis, M., \& Bosman, W. (2004). The prevention and treatment of overweight and obesity: summary of the advisory report by the Health Council of the Netherlands. The Netherlands Journal of Medicine, 62, 10-17.

- Kerr, J., Eves, F.F., \& Carroll, D. (2000). Posters can prompt less active individuals to use the stairs. Journal of Epidemiology and Community Health, 54, 942-943.

- Kerr, J., Eves, F.F., \& Carroll, D. (2001a). Six-month observational study of prompted stair climbing. Preventive Medicine, 33, 422-427.

- Kerr, J., Eves, F.F., \& Carroll, D. (2001b). The influence of poster prompts on stair use: the effects of setting, poster size and content. British Journal of Health Psychology, 6, 397-405.

- Kerr, J., Eves, F.F., \& Carroll, D. (2001c). Encouraging stair use: banners are better than posters. American Journal of Public Health, 91, 1192-1193.

- Kerr, J., Eves, F.F., \& Carroll, D. (2001d). Getting more people on the stairs: the impact of a new message format. Journal of Health Psychology, 6, 495-500.

- Kerr, J., Eves, F.F., \& Carroll, D. (2001e). Can posters prompt stair use in a worksite environment? Journal of Occupational Health, 43, 205-207.

- Kerr, N.A., Yore, M.M., Ham, S.A., \& Dietz, W.H. (2004). Increasing stair use in a worksite through environmental changes. American Journal of Health Promotion, 18, 312-315.

- Kim, S., \& Popkin, B.M. (2006). Commentary: understanding the epidemiology of overweight and obesity-a real global public health concern. International Journal of Epidemiology, 35, 60-67, discussion 81-82. 
- Klem, M.L., Viteri, J.E., \& Wing, R.R. (2000). Primary prevention of weight gain for women aged 25-34: the acceptability of treatment formats. International Journal of Obesity, 24, 219-225.

- Kipnis, V., Carroll, R.J., Freedman, L.S., \& Li, L. (1999). Implications of a New Dietary Measurement Error Model for Estimation of Relative Risk: Application to Four Calibration Studies. American Journal of Epidemiology, 150, 642-651.

- Koh-Banerjee, P., Chu, N.F., Spiegelman, D., Rosner, B., Colditz, G., Willet, W., \& Rimm, E. (2003). Prospective study of the association of changes in dietary intake, physical activity, alcohol consumption, and smoking with 9-y gain in waist circumference among 16587 US men. American Journal of Clinical Nutrition, 78, 719-727.

- Kral, T.V., \& Rolls, B.J. (2004). Energy density and portion size: their independent and combined effects on energy intake. Physiology and Behaviour, 82, 131-138.

- Krebs-Smith, S.M., Heimendinger, J., Patterson, B.H., Subar, A.F., Kessler, R., \& Pivonka, E. (1995). Psychosocial factors associated with fruit and vegetable consumption. American Journal of Health Promotion, 10, 98-104.

- Kremers, S.P.J., De Bruijn, G.J., Schaalma, H., \& Brug, J. (2004). Clustering of energy balance-related behaviours and their intrapersonal determinants. Psychology and Health, 19, 595-606.

- Kremers, S.P.J., Visscher, T.L., Brug, J., Chin A Paw, M.J., Schouten, E.G., Schuit, A.J., Seidell, J.C., van Baak, M.A., van Mechelen, W., Kemper, H.C., Kok, F.J., Saris, W.H., \& Kromhout, D. (2005a). Netherlands research programme weight gain prevention (NHF-NRG): rationale, objectives and strategies. European Journal of Clinical Nutrition, 59, 498-507.

- Kremers, S.P.J., de Bruijn, G.J., Droomers, M., van Lenthe, F., \& Brug, J. (2005b). Environmental interventions for selected dietary behaviours in adults. In:J. Brug, F. Van Lenthe (Eds), Environmental determinants and interventions for physical activity, nutrition and smoking: A review (pp. 282-314). Zoetermeer: Speed-Print b.v.

- Kremers, S.P.J., de Bruijn, G.J., Wendel-Vos, W., van Lenthe, F., \& Brug, J. (2005c). Environmental interventions on physical activity in adults. In: J. Brug, F. van Lenthe (Eds.), Environmental determinants and interventions for physical activity, nutrition and smoking: A review (pp. 140-164). Zoetermeer: Speed-Print b.v.

- Kremers, S.P.J., Visscher, T.L.S., Seidell, J.C., Van Mechelen, W., \& Brug, J. (2005d). Cognitive determinants of energy balance-related behaviours: measurement issues. Sports Medicine, 35, 923-933.

- Kremers, S.P.J., de Bruijn, G.J., Visscher, T.L.S., van Mechelen, W., de Vries, N. \& Brug, J. (2006). Environmental influences on energy balance-related behaviours: a dualprocess view. International Journal of Behavioural Nutrition and Physical Activity, 3, 9.

- Kroeze, W., Werkman, A. \& Brug, J. (2006). A systematic review of randomized trials on the effectiveness of computer-tailored education on physical activity and dietary behaviors. Annals of Behavioral Medicine, 31, 205-223.

- Kumanyika, S.K. (2001). Minisymposium on obesity: overview and some strategic considerations. Annual Review of Public Health, 22, 293-308.

- Kumanyika, S., Jeffery, R.W., Morabia, A., Ritenbaugh, C., \& Antipatis, V.J. (2002). Obesity prevention: the case for action. International Journal of Obesity, 26, 425-436.

- Kwak, L., Kremers, S.P.J., van Baak, M.A., \& Brug, J. (2006a). Participation rates in worksite-based intervention studies: health promotion context as a crucial quality criterion. Health Promotion International, 21, 66-69.

- Kwak, L., Kremers, S.P.J., Walsh, A., \& Brug, J. (2006b). How is your walking group running? Health Education, 106, 21-31.

- Kwak, L., Kremers, S.P.J., van Baak, M.A., \& Brug,J.(2007a). Formation of implementation intentions promotes stair use. American Journal of Preventive Medicine, 32, 254-255. 
- Kwak, L., Kremers, S.P.J., Werkman, A., Visscher, T.L.S., Van Baak, M.A., \& Brug, J. (2007b). The NHF-NRG In Balance-project: the application of Intervention Mapping in the development, implementation and evaluation of weight gain prevention at the worksite. Obesity Reviews, 8, 347-61.

- Lahtinen, E., Koskinen-Ollonqvist, P., Rouvinen-Wilenius, P., Tuominen, P., \& Mittelmark, M.B. (2005). The development of quality criteria for research: a Finnish approach. Health Promotion International, 20, 306-315.

- Landis, J.R., \& Koch, G.G. (1977). The measurement of observer agreement for categorical data. Biometrics, 33, 159-174.

- Larsen, K.S., Martin, H.J., Ettinger, R.H., \& Nelson, J. (1976). Approval seeking, social cost, and aggression: a scale and some dynamics. Journal of Psychology, 94, 3-11.

- Larsen, P. \& Simons, N. (1993) Evaluating a federal health and fitness program: indicators of improving health. Official Journal of the American Association of Occupational Health Nurses, 41,143-148.

- Lechner, L., Brug, J., \& de Vries, H. (1997). Misconceptions of fruit and vegetable consumption: differences between objective and subjective estimation of intake. Journal of Nutrition Education and Behaviour, 29, 313-320.

- Ledikwe, J.H., Ello-Martin, J.A., \& Rolls, B.J. (2005). Portion sizes and the obesity epidemic. Journal of Nutrition, 135, 905-909.

- Leenders, N.Y., Sherman, W.M., Nagaraja, H.N., \& Kien, C.L.. (2001). Evaluation of methods to assess physical activity in free living condition. Medicine and Science in Sports and Exercise, 33, 1233-1240.

- Leenders, N.Y., Sherman, W.M., \& Nagaraja, H.N. (2006). Energy expenditure estimated by accelerometry and doubly labeled water: do they agree? Medicine and Science in Sports and Exercise, 38, 2165-72.

- Lemieux, S., Prud'homme, D., Bouchard, C., Tremblay, A., \& Després, J. (1996). A single threshold value of waist girth identifies normal-weight and overweight subjects with excess visceral adipose tissue. American Journal of Clinical Nutrition, 64, 685-693.

- Leventhal, H., Singer, R., \& Jones, S. (1965). Effects of fear and specificity of recommendation upon attitudes and behavior. Journal of Personality and Social Psychology, 2, 20-29.

- Lewis, C.E., Jacobs, D.R. Jr., McCreath, H., Kiefe, C.I., Schreiner, P.J., Smith, D.E., \& Williams, O.D. (2000). Weight gain continues in the 1990s: 10-year trends in weight and overweight from the CARDIA study. Coronary Artery Risk Development in Young Adults. American Journal of Epidemiology, 151, 1172-1181.

- Lewis, D. (1998). The Internet as a resource for healthcare information. Diabetes Education, 24, 627-630, 632.

- Lingwood, P. (2004). Promoting walking and cycling as an alternative to using cars: perception of safety is biggest obstacle. British Medical Journal, 329, 1237, author reply 1238.

- Linnan, L.A., Sorensen, G., Colditz, G., Klar, N., \& Emmons, K.M. (2001). Using theory to understand the multiple determinants of low participation in worksite health promotion programs. Health Education \& Behaviour, 28, 591-607.

- Livingstone, M.B., Robson, P.J., Wallace, J.M., \& McKinley, M.C. (2003). How active are we? Levels of routine physical activity in children and adults. The Proceedings of the Nutrition Society, 62, 681-701.

- Lippke, S., Ziegelmann, J.P., \& Schwarter, R. (2004). Behavioural intentions and action plans promote physical exercise: a longitudinal study with orthopaedic rehabilitation patients. Journal of Sport and Exercise Psychology, 26, 470-483.

- Lipsey, M.W. (1990). Design sensitivity: Statistical power for experimental design. Sage Publications, Thousand Oaks, CA. 
- Ma, J., Betts, N.M., Horacek, T., Georgiou, C., White, A., \& Nitzke, S. (2002). The importance of decisional balance and self-efficacy in relation to stages of change for fruit and vegetable intakes by young adults. American Journal of Health Promotion, 16, 157-166.

- Marshall, A.L., Bauman, A.E., Patch, C., Wilson, J., \& Chen, J. (2002). Can motivational signs prompt increases in incidental physical activity in an Australian health-care facility? Health Education Research, 17, 743-749.

- Martens, M.K., Van Assema, P., Paulussen, G.W., Schaalma, H., \& Brug, J. (2006). Krachtvoer: process evaluation of a Dutch programme for lower vocational schools to promote healthful diet. Health Education Research, 21, 695-704.

- Mason, C. (2000). Transport and health: en route to a healthier Australia? Medical Journal of Australia, 172, 230-232.

- Matson-Koffman, D.M., Brownstein, J.N., Neiner, J.A., \& Greaney, M.L. (2005). A sitespecific literature review of policy and environmental interventions that promote physical activity and nutrition for cardiovascular health: what works? American Journal of Health Promotion, 19, 167-193.

- McLean, N., Griffin, S., Toney, K., \& Hardeman, W. (2003). Family involvement in weight control, weight maintenance and weight-loss interventions: a systematic review of randomised trials. International Journal of Obesity, 27, 987-1005.

- Miller, W.C. (1999). How effective are traditional dietary and exercise interventions for weight loss? Medicine and Science in Sports and Exercise, 31, 1129-1131.

- Milne, S., Orbell, S., \& Sheeran, P. (2002). Combining motivational and volitional interventions to promote exercise participation: protection motivation theory and implementation intentions. British Journal of Health Psychology, 7, 163-184.

- Moher, D., Schulz, K.F., \& Altman, D.G. (2001). The CONSORT statement: revised recommendations for improving the quality of reports of parallel-group randomized trails. Lancet, 357, 1191-1194.

- Moller, L.F., Kristensen, T.S., \& Hollnager, H. (1991). Physical activity, physical fitness, and cardiovascular risk factors. Danish Medicial Bulletin, 38, 182-187.

- Mullis, R.M., Blair, S.N., Aronne, L.J., Bier, D.M., Denke, M.A., Dietz, W., Donato, K.A., Drewnowski, A., French, S.A., Howard, B.V., Robinson, T.N., Swinburn, B., \& Wechsler, H. (2004). Prevention Conference VII: Obesity, a worldwide epidemic related to heart disease and stroke: Group IV: prevention/treatment. Circulation, 110, 484-488.

- Mulvihill C., Quigley R. (2003). The management of obesity and overweight. An analysis of reviews of diet, physical activity and behavioural approaches. In: NHS Health Development Agency, 1-53.

- National Institute for Health and Clinical Excellence. Obesity: guidance on the prevention, identification, assessment and management of overweight and obesity in adults and children. NICE clinical guideline 43 (2006). Available from URL: http://www.nice.org.uk.

- National Institute of Health. NIH guide: Improving diet and physical activity assessment (2002). Available from URL: http://grant.nih.gov/grants/guide/pa-files/PAR-03-009.html.

- Norman, A., Bellocco, R., Bergstrom, A., \& Wolk, A. (2001). Validity and reproducibility of self-reported total physical activity-differences by relative weight. International Journal of Obesity, 25, 682-688.

- O’Brien, A., Fries, E., \& Bowen, D. (2000). The effect of accuracy of perceptions of dietary-fat intake on perceived risk and intentions to change. Journal of Behavioral Medicine, 23, 465-473.

- Oenema, A., Brug, J., \& Lechner, L. (2001). Web-based tailored nutrition education: results of a randomized controlled trial. Health Education Research, 16, 647-660.

- Oenema, A., \& Brug, J. (2003). Feedback strategies to raise awareness of personal dietary intake: results of a randomized controlled trial. Preventive Medicine, 36, 429-439. 
- Oja, P., Vuori, I., \& Paronen, O. (1998). Daily walking and cycling to work: their utility as health-enhancing physical activity. Patient Education and Counselling, 33, S87-S94.

- Oldenburg, B.F., Sallis, J.F., French, M.L., \& Owen, N. (1999). Health promotion research and the diffusion and institutionalization of interventions. Health Education Research, 14, (1), 121-130.

- Oldenburg, B., Sallis, J.F., Harris, D., \& Owen, N. (2002). Checklist of Health Promotion Environments at Worksites (CHEW): development and measurement characteristics. American Journal of Health Promotion, 16, 288-299.

- Orbell, S., Hodgkins, S., \& Sheeran, P. (1997). Implementation intentions and the theory of planned behaviour. Personality and Social Psychology Bulletin, 23, 945-954.

- Pelletier, K.R. (1999). A review and analysis of the clinical and cost-effectiveness studies of comprehensive health promotion and disease management programs at the worksite: 1995-1998 update (IV). American Journal of Health Promotion, 13, 333-345.

- Pelletier K.R. (2001). A review and analysis of the clinical and cost-effectiveness studies of comprehensive health promotion and disease management programs the worksite: 1998-2000 update. American Journal of Health Promotion, 16, 107-116.

- Perez-Rodrigo, C., Wind, M., Hildonen, C., Bjelland, M., Aranceta, J., Klepp, K.I., \& Brug, J. (2005). The pro children intervention: applying the intervention mapping protocol to develop a school-based fruit and vegetable promotion programme. Annals of Nutrition and Metabolism, 49, 267-277.

- Perry, C.L., Bishop, D.B., Taylor, G.L., Davis, M., Story, M., Gray, C. Bishop, S.C., Mays, R.A., Lytle, L.A., \& Harnack, L. (2004). A randomized school trial of environmental strategies to encourage fruit and vegetable consumption among children. Health Education \& Behaviour, 31, 65-76.

- Petty, R.E., \& Cacioppo, R.T. (1986). The elaboration likelihood model of persuasion. Advances in Experimental Social Psychology, 19, 124-205.

- Pikora, T., Giles-Corti, B., Bull, F., Jamrozik, K., \& Donovan R. (2003). Developing a framework for assessment of the environmental determinants of walking and cycling. Social Science in Medicine, 56, 1693-1703.

- Pi-Sunyer, F.X. (2000). Obesity: criteria and classification. The Proceedings of the Nutrition Society, 2000, 59, 505-509.

- Povey, R., Conner, M., Sparks, P., James, R., \& Shepherd, R. (2000). Application of the Theory of Planned Behaviour to two dietary behaviours: roles of perceived control and self-efficacy. British Journal of Health Psychology, 5, 121-139.

- Prentice, A.M., \& Jebb, S.A. (1995). Obesity in Britain: gluttony or sloth? British Medical Journal, 311, 437-439.

- Prestwich, A, Lawton, R., \& Conner, M. (2003). The use of implementation intentions and the decision balance sheet in promoting exercise behaviour. Psychology and Health, 18, 707-721.

- Prestwich, A., Conner, M., Lawton, R., Bailey, W., Litman, J., \& Molyneaux, V. (2005). Individual and collaborative implementation intentions and the promotion of breast self-examination. Psychology and Health, 20, 743-760.

- Prochaska, J.O., \& DiClemente, C.C. (1983). Stages and processes of self-change of smoking: Toward an integrative model of change. Journal of Consulting and Clinical Psychology, 51, 295-304.

- Proper, K., Hildebrandt, V., \& Urlings, I.(1999). Nederlandse beroepsbevolking mag meer bewegen. [Ducth blue-collar workers should exercise more] Arbeidsomstandigheden, 11, 38-43.

- Proper, K., Hildebrandt, V., Urlings, I., \& Eikhout, S. (2000). Trap eens wat vaker naar je baas, Fietsen naar het werk als remedie tegen bewegingsarmoede [You should cycle more often to your boss, Cycling to work as a solution for exercise shortage]. Arbeidsomstandigheden, 4, 40-43. 
- Rabash, J., Browne, W., Goldstein, H., Yang, M., Plewis I., Draper, D., Healy, M., \& Woodhoose, G. (1999). A user's guide to MlwiN. London: Institute of Education.

- Raben, A., Jensen, N.D., Marckmann, P., Sandstrom, B., \& Astrup, A. (1995). Spontaneous weight loss during 11 weeks' ad libitum intake of a low fat/high fibre diet in young, normal weight subjects. International Journal of Obesity, 19, 916-923.

- Resnicow, K., McCarty, F., Blissett, D., Wang, T., Heitzler, C., \& Lee, R.E. (2003). Validity of a modified CHAMPS physical activity questionnaire among African-Americans. Medicine and Science in Sports and Exercise, 35, 1537-1545.

- Richardson, M.T., Ainsworth, B.E., Jacobs, D.R., \& Leon, A.S. (2001). Validation of the Stanford 7-day recall to assess habitual physical activity. Annals of Epidemiology, 11, 145-153.

- Rimm, E.B., Stampfer, M.J., Giovannucci, E., Ascherio, A., Spiegelman, D., Colditz, G.A., \& Willett, W.C. (1995). Body size and fat distribution as predictors of coronary heart disease among middle-aged and older US men. American Journal of Epidemiology, 148, 1187-1194.

- Roberts, S.B., McCrory, M.A., \& Saltzman, E.(2002). The influence of dietary composition on energy intake and body weight. Journal of the American College of Nutrition, 21, S140S145.

- Rogers, E.M. (1995). Diffusion of innovations. New York: Free Press.

- Rolls, B.J. (2000). The role of energy density in the overconsumption of fat. Journal of Nutrition, 130, S268-S271.

- Rolls, B.J., \& Bell, E.A. (2000). Dietary approaches to the treatment of obesity. The Medical Clinics of North America, 84, 401-418.

- Rolls, B.J., Morris, E.L., \& Roe, L.S. (2002). Portion size of food affects energy intake in normal-weight and overweight men and women. American Journal of Clinical Nutrition, 76, 1207-1213.

- Rolls, B.J., Roe, L.S., Meengs, J.S., \& Wall, D.E. (2004). Increasing the portion size of a sandwich increases energy intake. Journal of American Dietetic Association, 104, 367-372.

- Rolls, B.J., Drewnowski, A., \& Ledikwe, J.H. (2005). Changing the energy density of the diet as a strategy for weight management. Journal of American Dietetic Association, 105, S98-S103.

- Rooney, B., Smalley, K., Larson, J., \& Havens, S. (2003). Is knowing enough? Increasing physical activity by wearing a pedometer. WMJ, 102, 31-36.

- Ross, R., Dagnone, D., Jones, P.J., Smith, H., Paddags, A., Hudson, R., \& Janssen, I. (2000). Reduction in obesity and related comorbid conditions after diet-induced weight loss or exercise-induced weight loss in men. A randomized, controlled trial. Annals of Internal Medicine, 133, 92-103.

- Rothacker, D.Q., \& Blackburn, G.L. (2000). Obesity prevalence by age group and 5year changes in adults residing in rural Wisconsin. Journal of the American Dietetic Association, 100, 784-790.

- Russell, W.D., Dzewaltowski, D.A., \& Ryan, G.J. (1999). The effectiveness of a point-ofdecision prompt in deterring sedentary behavior. American Journal of Health Promotion, 13, 257-259.

- Russell, W.D., \& Hutchinson, J. (2000). Comparison of health promotion and deterrent prompts in increasing use of stairs over escalators. Perceptual and Motor Skills, 91, 55-61.

- Sallis, J.F., Bauman, A., \& Pratt, M. (1998). Environmental and policy interventions to promote physical activity. American Journal of Preventive Medicine, 15, 379-397. 
- Sarria, A., Garcia-Llop, L.A., Moreno, L.A., Fleta, J., Morellon, M.P., \& Bueno, M. (1998). Skinfold thickness measurements are better predictors of body fat percentage than body mass index in male Spanish children and adolescents. European Journal of Clinical Nutrition, 52, 573-576.

- Sarlio-Lahteenkorva, S., \& Lahelma, E. (1999). The association of body mass index with social and economic disadvantage in women and men. International Journal of Epidemiology, 28, 445-449.

- Schneider, S., \& Becker, S. (2005). Prevalence of physical activity among the working population and correlation with work-related factors: results from the first German National Health Survey. Journal of Occupational Health, 47, 414-423.

- Schoeller, D.A. (1990). How accurate is self-reported dietary energy intake? Nutrition Reviews, 48, 373-379.

- Schoeller, D.A., \& Delany, J.P. (1998). Human energy balance: what have we learned from the doubly labelled water method? American Journal of Clinical Nutrition, 68, 927-979.

- Seidell, J.C., Deurenberg, P., \& Hautvast, J.G. (1987). Obesity and fat distribution in relation to health-current insights and recommendations. World Review of Nutrition and Dietetics, 50, 57-91.

- Seidell, J.C. (2000). The current epidemic of obesity. In: C. Bouchard (Ed) Physical Activity and Obesity. Human Kinetics, Campaign, IL, 21-30.

- Seidell, J.C., Perusse, L., Despres, J.P., \& Bouchard C.(2001). Waist and hip circumferences have independent and opposite effects on cardiovascular disease risk factors: the Quebec Family Study. American Journal of Clinical Nutrition, 74, 315-321.

- Seymour, J.D., Yaroch, A.L., Serdula, M., Blanck, H.M., \& Khan, L.K. (2004). Impact of nutrition environmental interventions on point-of-purchase behaviour in adults: a review. Preventive Medicine, 39, Suppl 2, 108-136.

- Shadish, W.R., Cook, T.D., \& Campbell, D.T. (2002). Quasi-experimental designs for generalized causal inference. Boston: Houghton Mifflin Company.

- Sheehan, T.J., DuBrava, S., DeChello, L.M., \& Fang, Z. (2003). Rates of weight change for black and white Americans over a twenty year period. International Journal of Obesity, 27, 498-504.

- Sheeran, P., \& Orbell, S. (1999). Implementation intentions and repeated behavior: augmenting the predictive validity of the theory of planned behavior. European Journal of Social Psychology, 29, 349-369.

- Sheeran, P., \& Orbell, S. (2000). Using implementation intentions to increase attendance for cervical cancer screening. Health Psychology, 19, 283-289.

- Sheeran, P., Aarts, H., Custers, R., Rivis, A., Webb, T.L., \& Cooke, R. (2005a). The goal-dependent automaticity of drinking habits. British Journal of Social Psychology, 44, 47-63.

- Sheeran, P., Webb, T.L., \& Gollwitzer, P.M. (2005b). The interplay between goal intentions and implementation intentions. Personality and Social Psychology Bulletin, 31, 87-98.

- Sheeran, P., Webb, T.L., \& Gollwitzer, P.M. (2006). Implementation Intentions: Strategic Automatization of Goal Striving. In: De D.T.D. Ridder \& De J.B.F. Wit (Eds). Self-regulation in Health Behaviour: John Wiley \& Sons, Ltd.

- Shephard, R.J. (1996). Worksite fitness and exercise programs: a review of methodology and health impact. American Journal of Health Promotion, 10, 436-452.

- Shephard, R.J. (2003). Limits to the measurement of habitual physical activity by questionnaires. Bristish Journal of Sports Medicine, 37, 197-206; discussion 206.

- Sherwood, N.E., Jeffery, R.W., French, S.A., Hannan, P.J., \& Murray, D.M. (2000). Predictors of weight gain in the Pound of Prevention study. International Journal of Obesity, 24, 395-403. 
- Simpson, J.M., Oldenburg, B., Owen, N., Harris, D., Dobbins, T., Salmon, A., Vita, P., Wilson, J., \& Saunders, J.B. (2000). The Australian National Workplace Health Project: design and baseline findings. Preventive Medicine, 31, 249-260.

- Singh, A.S., Chin A Paw, M.J., Kremers, S.P.J., Visscher, T.L.S., Brug, J., \& van Mechelen, W. (2006). Design of the Dutch Obesity Intervention in Teenagers (NRG-DOiT): systematic development, implementation and evaluation of a school-based intervention aimed at the prevention of excessive weight gain in adolescents. BMC Public Health, 6, 304.

- Skinner, B.F. (1963). Operant behaviour. American Psychologist, 18, 503-515.

- Slavin, J.L. (2005). Dietary fibre and body weight. Nutrition, 21, 411-418.

- Slentz, C.A., Duscha, B.D., Johnson, J.L., Ketchum, K., Aiken, L.B., Samsa, G.P., Houmard, J.A., Bales, C.W., \& Kraus, W.E. (2004). Effects of the amount of exercise on body weight, body composition, and measures of central obesity: STRRIDE a randomized controlled study. Archives of Internal Medicine, 164, 31-39.

- Smith, J.M., \& Bennett, R. (2002). Several antecedent strategies in reduction of an environmentally destructive behavior. Psychology Reports, 70, 241-242.

- Smith, A.T., Kuznesof, S., Richardson, D.P., \& Seal, C.J. (2003). Behavioural, attitudinal and dietary responses to the consumption of wholegrain foods. The Proceedings of the Nutrition Society, 62, 455-467.

- Sorensen, G., Thompson, B., Glanz, K., Feng, Z., Kinne, S., DiClemente, C., Emmons, K., Heimendinger, J., Probart, C., \& Lichtenstein E. (1996). Worksite based cancer prevention: primary results from the Working Well Trial. American Journal of Public Health, 86, 939-947.

- Sorensen, G., Stoddard, A., Hunt, M.K., Hebert, J.R., Ockene, J.K., Avrunin, J.S., Himmelstein, J., \& Hammond S.K. (1998). The effects of a health promotion-health protection intervention on behaviour change: the WellWorks Study. American Journal of Public Health, 88, 1685-1690.

- Sorensen, G., Stoddard, A., Peterson, K., Cohen, N., Hunt, M.K., Stein, E., Palombo, R., \& Lederman, R. (1999). Increasing fruit and vegetable consumption through worksites and families in the treatwell 5-a-day study. American Journal of Public Health, 89, 54-60. - Speakman, J.R. (2001). The history and theory of the doubly labelled water technique. American Journal of Clinical Nutrition, 68, 932-938.

- Sorensen, G., Linnan, L., \& Hunt, M.K. (2004). Worksite-based research and initiatives to increase fruit and vegetable consumption. Preventive Medicine, 39, S94-S100.

- Starling, R.D., Matthews, D.E., Ades, P.A., \& Poehlman, E.T. (1999). Assessment of physical activity in older individuals; a doubly labelled study. Journal of Applied Physiology, 86, 2090-2096.

- Stephenson, J., Bauman, A., Armstrong, T., Smith B., \& Bellew, B. (2000). The cost of illness attributable to physical inactivity in Australia, a preliminary study, CDHAC, Canberra.

- Stokols, D. (1992). Establishing and maintaining healthy environments: Toward a social ecology of health promotion. American Psychologist, 47, 6-22.

- Strath, S.J., Bassett, D.R. Jr., \& Swartz, A.M. (2003). Comparison of MTI accelerometer cut-points for predicting time spent in physical activity. International Journal of Sports Medicine, 24, 298-303.

- Swartz, A.M., Strath, S.J., Bassett, D.R. Jr., O’Brien, W.L., King, G.A., \& Ainsworth, B.E. (2000). Estimation of energy expenditure using CSA accelerometers at hip and wrist sites. Medicine and Science in Sports and Exercise, 32, S450-S456.

- Swinburn, B., Egger, G., \& Raza, F. (1999). Dissecting obesogenic environments: the development and application of a framework for identifying and prioritizing environmental interventions for obesity. Preventive Medicine, 29, 563-570.

- Teh, K.C., \& Aziz, A.R. (2002). Heart rate, oxygen uptake, and energy cost of ascending and descending the stairs. Medicine and Science in Sports and Exercise, 34, 695-699. 
- Thomsom, C.A., Giuliano, A., Rock, C.L., Ritenbaugh, C.K., Flatt, S.W., Faerber, S., Newman, V., Caan, B., Graver, E., Hartz ,V., Whitacre, R., Parker, F, Pierce, J.P., \& Marshall, J.R. (2003). Measuring Dietary Change in a Diet Intervention Trial: Comparing Food Frequency Questionnaire and Dietary Recalls. American Journal of Epidemiology, 157, 754-762.

- Timperio, A., Salmon, J., \& Crawford, D. (2003). Validity and reliability of a physical activity recall instrument among overweight and non-overweight men and women. Journal of Science and Medicine in Sport, 6, 477-491.

- Tinker, L.F., Patterson, R.E., Kristal, A.R., Bowen, D.J., Kuniyuki, A., Henry, H., \& Shattuck, A. (2001). Measurement characteristics of 2 different self-monitoring tools used in a dietary intervention study. Journal of American Dietetic Association, 101, 1031-1040.

- Titze, S., Martin, B.W., Seiler, R., \& Marti, B. (2001). A worksite intervention module encouraging the use of stairs: results and evaluation issues. Sozial-und Präventivmedizin, 46, 13-19.

- Tones, K. (2000). Evaluating health promotion-beyond the RCT. In L. Norheim \& M. Waller (Eds.). Best Practices, Quality and Effectiveness of Health Promotion (pp. 86-101). Helsinki, Finland: Finnish Centre for Health Promotion.

- Triandis, H.C. (1977). Interpersonal behaviour. Monterey, CA: Brooks/Cole.

- Trost, S.G., Owen, N., Bauman, A.E., Sallis, J.F., \& Brown W. (2002). Correlates of adults' participation in physical activity: review and update. Medicine and Science in Sports and Exercise, 34, 1996-2001.

- Urlings I., Hildebrandt, V., van Lingen P., \& Lourijsen, E. (1997). Nederlandse werknemer beweegt alleen onder voorwaarde(n) [Dutch employee only moves under conditions]. Arbeidsomstandigheden, 4, 191-193.

- US Department of Health and Human Services: 1992 National Survey of Worksite Health Promotion Activities: Summary Report. (1993). Washington, DC: U.S. Government Printing Office.

- Van Assema, P., Brug, J., Dolders, M., Kok, G., \& Steenhuis, I. (1996). Misconceptie van vetconsumptie. Een kwalitatief onderzoek naar oorzaken. [Misconception of fat consumpion: A qualitative study of causes]. Tijdschrift voor gezondheidswetenschappen, 74, 347-355.

- Van Assema, P., Brug, J.,\& Lechner, L. (2001). Voedingsgedrag en de primaire preventie van kanker: de promotie van groente en fruit [Dietary behaviour and the primary prevention of cancer: the promotion of fruit and vegetables]. Tijdschrift Sociale Geneeskunde, 6, 346-355.

- Vanden Auweele, Y., Boen, F., Schapendonk, W., \& Dornez, K. (2005). Promoting stair use among female employees: the effects of a health sign followed by an e-mail. Journal of Sports Exercise Psychology, 27, 188-196.

- Van Duyn, M.A., Kristal, A.R., Dodd, K., Campbell, M.K., Subar, A.F., Stables, G., Nebeling, L., \& Glanz, K. (2001). Association of awareness, intrapersonal and interpersonal factors, and stage of dietary change with fruit and vegetable consumption: a national survey. American Journal of Health Promotion, 16, 69-78.

- Van Empelen, P., Kok, G., Schaalma, H., \& Bartholomew, L.K. (2003). An aids risk reduction program for Dutch drug users: an intervention mapping approach to planning. Health Promotion Practice, 4, 402-412.

- Verplanken, B., \& Faes, S. (1999). Good intentions, bad habits, and effects of forming implementation intentions on healthy eating. European Journal of Social Psychology, 29, 591-604. 
- Visscher, T.L.S., Seidell, J., Molarius, A., van der Kuip, D., Hofman A., \& Witteman, J. (2001). A comparison of body mass index, waist-hip ration and waist circumference as predictors of all-cause mortality among the elderly: the Rotterdam study. International Journal of Obesity, 25, 1730-1735.

- Visscher, T.L.S., Kromhout, D., \& Seidell, J.C. (2002). Long-term and recent time trends in the prevalence of obesity among Dutch men and women. International Journal of Obesity, 26, 1218-1224.

- Wammes, B., Kremers, S., Breedveld, B., \& Brug, J. (2005). Correlates of motivation to prevent weight gain: a cross sectional survey. International Journal of Behavioural Nutrition and Physical Activity, 2, 1.

- Wammes, B., French, S., \& Brug, J. (2007). What young Dutch adults say they do to keep from gaining weight: self-reported prevalence of overeating, compensatory behaviours and specific weight control behaviours? Public Health Nutrition, 7, 1-9

- Wardle, J., Parmenter, K., \& Waller, J. (2000). Nutrition knowledge and food intake. Appetite, 34, 269-275.

- Wareham, N.J., van Sluis, E.M., \& Ekelund, U. (2005). Physical activity and obesity prevention: a review of the current evidence. The Proceedings of the Nutrition Society, 64, 229-247.

- Webb, O.J., \& Eves, F.F. (2005). Promoting stair use: single versus multiple stair-riser messages. American Journal of Public Health, 95, 1543-1544.

- Webb, O.J., \& Eves, F.F. (2007). Promoting stair climbing: effects of message specificity and validation. Health Education Research, 22, 49-57.

- Weber, J., Tilford, J., Szeto, K., \& Wheeler, G. (2005). Economic evaluation of a pricing strategy to alter vending machine selections of employees in a rural worksite. In: A. Oenema, W. Kroeze, J. Brug (Eds). Fourth Annual Conference of the International Society of Behavioural Nutrition and Physical Activity (pp. 77). Amsterdam.

- Weinstein, N.D. (1988). The Precaution Adoption Process. Health Psychology, 7, 355-386.

- Welk, G.J., Blair, S.N., Wood, K., Jones, S., \& Thompson, R.W. (2000). A comparative evaluation of three accelerometry-based physical activity monitors. Medicine and Science in Sports and Exercise, 32, S489-S497.

- Wells, J.C.K., \& Victoria, C.G. (2005). Indices of whole-body and central adiposity for evaluating the metabolic load of obesity. International Journal of Obesity, 29, 483-489.

- Wen, L.M., Orr, N., Bindon, J., \& Rissel C. (2005). Promoting active transport in a workplace setting: evaluation of a pilot study in Australia. Health Promotion International, 20, 123-133.

- Wendel-Vos, G.C., Schuit, A.J., Saris, W.H., \& Kromhout, D. (2003). Reproducibility and relative validity of the short questionnaire to assess health-enhancing physical activity. Journal of Clinical Epidemiology, 56, 1163-1169.

- Werkman, A., Schuit, A.J., Kwak, L., Kremers, S.P.J., Visscher, T.L.S., Kok, F.J., \& Schouten, E.G. (2006). Study protocol of a cluster randomised controlled trial investigating the effectiveness of a tailored energy balance programme for recent retirees. BMC Public Health, 6, 293.

- Williamson, D.F., Kahn, H.S., Remington, P.L., \& Anda, R.F.(1990). The 10-year incidence of overweight and major weight gain in US adults. Archive of Internal Medicine, 150, 665-672.

- Wilmore, J.H., Després, J.P., Stanforth, P.R., Mandel, S., Rice, T., Gagnon, J., Leon, A.S., Rao, D., Skinner, J.S., \& Bouchard C. (1999). Alterations in body weight and composition consequent to 20 wk of endurance training: the HERITAGE family study. American Journal of Clinical Nutrition, 70, 346-352. 
- Winnet, R.A., Tate, D.F., Anderson, E.S., Wojcik, J.R., \& Winett, S.G. (2005). Long-term weight gain prevention: A theoretically based internet approach. Preventive Medicine, 41, 629-641.

- World Health Organization Consultation on Obesity (2000). Obesity: preventing and managing the global epidemic (WHO technical report series, 894). Geneva: World Health Organization.

- World Health Organization (2004). Global Strategy on Diet, Physical Activity and Health, Resolution of the Fifty-seventh World Health Assembly. Geneva: World Health Organization.

- Yao, M., \& Roberts, S.B. (2001). Dietary energy density and weight regulation. Nutrition Reviews, 59, 247-258.

- Yancey, A.K., McCarthy, W.J., Taylor, W.C., Merlo, A., Gewa, C., Weber, M.D., \& Fielding, J.E. (2004). The Los Angeles Lift Off: a sociocultural environmental change intervention to integrate physical activity into the workplace. Preventive Medicine, 38, 848-856.

- Ziegelmann, J.P., Lippke, S., \& Schwarzer R. (2006). Adoption and maintenance of physical activity: planning interventions in young, middle-aged, and older adults. Psychology and Health, 21, 145-163. 
SUMMARY 
This thesis reports on several studies related to the development, implementation and evaluation of the NHF-NRG In Balance-project, a worksite-based prevention program directed at the prevention of weight gain in young adults, through changes in both physical activity and food intake.

Chapter 1, describes the development, implementation and evaluation framework of the NHF-NRG In Balance-project following the steps of the Intervention Mapping protocol. The program was aimed at young adults as young adulthood is recognized as a high-risk period for weight gain, with an estimated annual weight gain of approximately $0.60 \mathrm{~kg} /$ year. In order to prevent the proposed weight gain the following weight gain preventive behaviours (WGPBs) were identified: increasing the frequency and duration of walking and cycling for transport; increasing the physical activity level at work; decreasing portion sizes and reducing the energy density of ingested food during the day. An intervention was developed combining environmental and individually tailored communications with worksite environmental changes to raise awareness, to motivate and to enable energy balance behaviour changes. In order to determine the efficacy of the intervention a quasi-experimental pre-test-multiple post-test control group design was applied in 12 worksites.

The original design included randomization, however as a result of recruitment problems that occurred before implementation of the program, worksites were not randomly assigned to the experimental and control group, but matched. Chapter 2 further elaborates on this issue by advocating the importance of the reporting of participation rates among approached worksites in publications on worksite intervention effects. Such information will help to draw conclusions on the practical relevance of the shown effectiveness of the intervention.

Chapter 3 reports on a study conducted to explore the accuracy of the questionnaire used to assess the outcome measure of the physical activity related WGPBs. The study compared the questionnaire (SQUASH), a $24 \mathrm{~h}$ recall measure (PAS) and accelerometer in a field setting during 7 days in a subgroup of the participants of the NHF-NRG In Balance-project. Significant correlations were observed between PAS and accelerometer in the overall population, men, normal weight and overweight participants. Correlations between SQUASH and accelerometer were only significant for men and overweight individuals. Moderate agreement was observed between tertiles of accelerometer and PAS activity scores in the overall population, but not between accelerometer and SQUASH. It was concluded that a 7-day administration of the PAS is a sufficiently useful measure for level and classification of physical activity, in the overall population. The SQUASH has practical advantages in terms of convenience of administrating, but its usefulness in estimating physical activity levels is limited.

Three studies within the thesis were conducted to test the effectiveness of one of the intervention components of the program, namely promoting stair use. Chapter 4 presents the results of a study investigating whether forming an implementation intention specifying stair use is effective in stimulating this behaviour. During the annual anthropometric measurements participants of one of the participating worksites were asked to form an implementation intention specifying either stair use (intervention group) or cycling in leisure time (control group). Directly after the measurements participants' stair or elevator use was observed. Of the intervention condition $83.3 \%$ used the stairs in comparison to $66.7 \%$ of the control condition. Stair use was influenced by a positive attitude toward stair use as a means to watch one's weight and a lower BMI $\left(\mathrm{kg} / \mathrm{m}^{2}\right)$. Forming an implementation intention 
specifying stair use had an additional effect.

The study presented in Chapter 5 further evaluated the effectiveness of the implementation intention on stair use but over a 12-month period. Twelve months after the formation of the implementation intentions, participants returned to the measurements and reported if they had taken the stairs or elevator to the examination room. Individuals in the intervention condition were more likely to take the stairs 12 months later than control individuals, however only among individuals who were motivated at baseline to be more active at work.

Chapter 6 reports on another method to increase stair use, namely the use of prompts on posters. The study tested whether an intervention using prompts, was effective in stimulating stair use in two of the participating worksites; one consisting mainly of white-collar workers and one mainly of blue-collar workers. The study used a simple time-series design of collecting data in three waves: before, during and after implementation of posters containing prompts stimulating stair use. A total of 6771 choices between stairs and elevator were observed. There was a significant difference between stair use at baseline and during the poster intervention in both types of worksites. There was no worksite-by-intervention interaction, implying that the prompts were equally effective in both types of worksites. After removal of the posters stair use decreased significantly to a level that was not significantly different from baseline. Stair use can be positively influenced in both blue- and white-collar workers by a short-term low-cost intervention using prompts on posters.

The two studies described in the last two chapters, examined the short-term and longer term effectiveness of the program. Chapter 7 reports on the effectiveness with regard to changes in sum of skinfolds, waist circumference, body weight and BMI 1 and 2 years after baseline The results indicated that with regard to changes in sum of skinfolds and waist circumference the project was indeed effective both at 12 and 24 months. Even though changes in weight and BMI between the intervention and control group were not significantly different, they did change in the desired direction. Overall, the intervention of the NHF-NRG In Balance-project had a positive effect on the body composition measures of the individuals in the intervention group.

The study described in Chapter 8 examined the effectiveness of the program with regard to the specified WGPBs and their corresponding cognitions. The results showed that nearly all WGPBs in the intervention group improved more than in the control group, even though improvements in behaviours were often too small to reach statistical significance. The results also showed that most observed effect sizes remained stable or even increased during the follow-up year. We were unable to explain the intervention effects on the WGPBs by changes in the hypothesised cognitive mediators, as no positive intervention effects were observed for the cognitive factors. In conclusion, the NHF-NRG In Balance-project resulted in small to medium effects in nearly all dietary intake and physical active behaviours.

The final chapter of the thesis (Chapter 9) is a general discussion. It is concluded that the systematic development of the NHF-NRG In Balance-project has resulted in a programme, which had a positive effect on the body composition, food intake and physical activity of the participants of the intervention group. However, it should be taken into consideration that as a result of the recruitment problems a group of worksites participated that was not representative of general worksites. This thesis does however give relevant information regarding the effectiveness of the NHFNRG In Balance-project in daily 'real-life'setting. 
SAMENVATTING 
In dit proefschtift worden verschillende studies beschreven, die elk gerelateerd zijn aan de ontwikkeling, implementatie en evaluatie van het NHS-NRG In Balans-project. Het NHS-NRG In Balans-project is een werkplek gebaseerd preventie programma dat zich richt op het voorkomen van gewichsstijging bij jong volwassenen, door veranderingen in lichamelijke activiteit en voeding te stimularen.

Hoofdstuk 1, beschrijft aan de hand van de stappen van het Interventie Mapping protocol het ontwikkelings, implementatie en evaluatie plan van het NHS-NRG In Balans-project. Het programma richtte zich op jong volwassenen, aangezien jong volwassenen een hoog risico groep vormen voor gewichsstijging, met een geschatte jaarlijkse gewichtsstijging van ongeveer $0.6 \mathrm{~kg}$. Aan de hand van de volgende gedragingen is gepoogd om gewichtsstijging te voorkomen: voorhogen van de frequentie en duur van wandelen en fietsen als transport; verhogen van de hoeveelheid lichamelijke activiteit op het werk; verkleinen van portie groottes en het verminderen van de energie dichtheid van de ingenomen voeding gedurende de dag. Het programma bestond uit verschillende interventies zowel gericht op het individu als de omgeving, met als doel het verhogen van bewustzijn en motivatie en het bewerkstelligen van de gespecifeerde gedragingen. Een quasi-experimenteel pretest-meervoudige post-test control groep design was toegepast om de effectiviteit van de interventie te bepalen.

Het oorspronkelijke onderzoeksdesign bestond uit randomisatie, echter als een gevolg van wervingsproblemen die optraden voor de implementatie van het programma, zijn de deelnemende bedrijven niet at random toegewezen aan de interventie of controle groep, maar gematched. Hoofdstuk 2, gaat hier verder op in en ondersteept het belang van het rapporteren van wervingsproblematiek in publicaties over werkplek interventies. Aan de hand van deze informatie kunnen verdere conclusies worden getrokken over de practische relevantie van de effectiviteit van interventies.

In Hoofdstuk 3, wordt een studie beschreven naar de nauwkeurigheid van een van de vragelijsten die gebruikt is binnen het NHS-NRG In Balans-project, namelijk de SQUASH welke als doel heeft lichamelijke activieit te meten. Gedurende een periode van 7-dagen is de SQUASH vergeleken met een 24 uurs maat (PAS) en een accelerometer in een dagelijkse setting binnen een subgroep van de deelnemers van het NHSNRG In Balans-project. Significante correlaties werden gevonden tussen de PAS en accelerometer, in de gehele groep, mannen, deelnemers met een normaal gewicht en deelnemers met overgewicht. Correlaties tussen de SQUASH en accelerometer waren echter alleen significant voor mannen en deelnemers met overgewicht. Binnen de totale groep lieten de resulaten een gematigde overeenkomst zien tussen de tertielen van de accelerometer en PAS activiteit scores, maar niet tussen de accelerometer en de SQUASH activiteit scores. Geconcludeerd werd dat een 7-daagse toepassing van de PAS een bruikbare is voor het classificeren van lichamelijke acitiviteit in de gehele deelnemende populatie. De SQUASH heeft voornamelijk practische voordelen wat betreft de toepassing, maar is minder nauwkeurig in het bepalen van lichamelijke activiteit niveaus.

Drie studies binnen het proefschrift zijn uitgevored om de effectiviteit van een van de interventieonderdelen te toetsen en wel het stimuleren van trap gebruik. Hoofdstuk 4 beschrijft de resultaten van een studie waarbij onderzocht werd of het vormen van een implementatie intentie gericht op trapgebruik effectief is in het stimuleren van trapgebruik. Gedurende de jaarlijkse anthropometrische metingen werden de deelnemers van een van de deelnemende bedrijven gevraagd om een implementatie intentie te vormen, gericht op trapgebruik (interventie groep) of op fietsen in de vrije tijd (controle groep). Na afloop van de de metingen werd gekeken 
of de deelnemers de trap of de lift namen. Van de interventie groep bleek 83.3\% met de trap te gaan in vergelijking met $66.7 \%$ van de controle groep. Het trapgebruik werd beinvloed door een positieve attitude ten opzichte van trapgebruik als een methode om op het gewicht te letten en een lage BMI $\left(\mathrm{kg} / \mathrm{m}^{2}\right)$. Het vormen van een implementatie intentie had een extra effect.

De studie in Hoofdstuk 5 evalueerde de effectiviteit van de implementatie intentie op trapgebruik na 12 maanden. Twaalf maanden na het vormen van de implemementatie intentie keerden de deenemers terug voor hun jaarlijkse metingen. Tijdens de metingen werd hen gevraagd of zij met de trap of lift naar de metinen waren gekomen. Twaalf maanden na het vormen van de implementatie intentie waren de deelnemers van de interventie groep meer geneigd om de trap te nemen dan deelnemers van de controle groep, dit was echter alleen het geval voor deelnemers die twaalf maanden eerder hadden aangegeven gemotiveerd te zijn om meer te gaan bewegen op het werk.

Hoofdstuk 6 beschrijft een andere methode om trapgebruik te stimuleren, namelijk prompts op posters. In deze studie is onderzocht of de interventie met prompts effectief was in het stimuleren van trapgebruik in twee deelnemende bedrijven, een met voornamelijk fabrieksarbeiders en een met voornamelijk kantoormedewerkers. Data werden verzameld op 3 momenten: voor, gedurende en na implementatie van de posters. In totaal zijn 6771 keuzes om de trap of lift te nemen waargenomen. In beiden bedrijven was er een significant verschil tussen het trapgebruik op baseline en gedurende de poster interventie. Er was echter geen werkplek-interventie interactie, wat suggereert dat de prompts even effectief waren in beiden bedrijven. Na het verwijderen van de posters nam het trapgebruik significant af naar een niveau dat niet significant verschillend was van baseline. Deze korte-termijn interventie was in staat om het trapgebruik van zowel fabrieksarbeiders als kantoormedewerkers positief te beinvloeden.

De twee studies die in de laatste twee hoofdstukken worden besproken onderzochten het korte-en lange termijn effect van het NHS-NRG In Balans-project. Hoofdstuk 7 beschrijft de effectiviteit wat betreft veranderingen in de som van de huidplooien, middelomtrek, gewicht en BMI 1 en 2 jaar na baseline. De resultaten laten zien dat het project effectief was wat betreft de veranderingen in som van de huidplooien en middelomtrek zowel na 12 en 24 maanden. Er waren geen significante veranderingen waar te nemen in gewicht en BMI tussen de twee groepen. Ongeacht dat deze veranderingen niet significant verschilde, veranderden zij wel in de gewenste richting. De interventie van het NHS-NRG In Balans-project had dus een positief effect op de lichaamsamenstelling van de deelnemers van de interventie groep.

De studie beschreven in Hoofdstuk 8 onderzocht de effectiviteit van het programma wat beterft de gespecifeerde gedragingen om gewichtsstijging te voorkomen en hun gerelateerde cognities. De resultaten lieten zien dat bijna alle gedragingen in de interventiegroep verbeterden, ondanks dat de veranderingen vaak te klein waren om statistische significantie te bereiken. De resultaten lieten tevens zien dat de meeste waargenomen 'effect sizes' stabiel bleven of zelfs toenamen gedurende het follow-up jaar. De interventie effecten konden niet verklaard worden aan de hand van de cognitieve mediatoren, aangezien er geen positief interventie effect waargenomen werd voor de cognitieve factoren. Concluderend kan gesteld worden dat het NHS-NRG In Balans-project heeft geresulteerd in kleine tot middel grote effecten in bijna alle voedings en beweeggedragingen.

Het proefschrift sluit af met een algemene discussie (Hoofdstuk 9). De algemene conclusie is dat de systematische ontwikkeling van het NHS-NRG In Balans-project heeft geleid tot een programma dat een positief effect heeft gehad op de lichaamssamenstelling en voedings en beweeggedragingen van de deelnemers 
van de interventie groep. Echter moet wel in acht worden genomen dat als gevolg van wervingsproblemen er mogelijk een niet representatieve groep bedrijven heeft deelgenomen. Dit proefschrift geeft waardevolle informatie over de effectiviteit van het NHS-NRG In Balans-project in een dagelijkse 'real-life'setting. 
DANKWOORD 
Dit proefschrift was niet tot stand gekomen zonder de hulp en steun van een hoop mensen, ik wil iedereen hiervoor dan ook heel erg bedanken! Stef, jou wil ik graag als eerste bedanken. Een betere begeleider had ik niet kunnen wensen, bedankt voor al je steun, motivatie en begeleiding! Ik hoop dat we nog lang contact zullen houden. Hans, bedankt voor je vertrouwen in mij, dit heeft me zeker tijdens de moeilijkere periode van het project altijd weer weten te motiveren om door te gaan. Marleen, bedankt dat je me de ruimte hebt gegeven om me binnen HB als GVO-er te ontwikkelen.

De beoodelingscommissie, bestaande uit Ellen Blaak, Nanne de Vries, Wim Saris, Michael Sjöström en Willen van Mechelen, wil ik graag bedanken voor de tijd en moeite die zij hebben gestopt in het lezen en beoordelen van mijn proefschrift. Michael, thank you for accepting the invitation to be part of the corona during the public defense of my thesis and for giving me the oppotunity to work at Prevnut.

Chantal, jou wil ik in het bijzonder bedanken voor al jouw hulp tijdens de uitvoering van het project. Zonder jou was het niet gelukt en een ding is zeker het was een stuk minder leuk geweest, ik zal onze vroege metingen en autoritjes niet vergeten! Ook heb ik dankbaar gebruik mogen maken van de hulp van verschillende stagiaires, die ik hierbij dan ook graag wil bedanken voor hun inzet.

Veel dank ben ik verschuldigd aan het AZM, Belden, Ciba Chemicals, Essent, Gemeente Nederweert, Gemeente Weert, Leolux, Sappi, Sint Jans Gasthuis, Trega International, Universiteit Maastricht, Watermaatschappij Limburg en hun werknemers. Zonder jullie had ik dit onderzoek niet kunnen uitvoeren, bedankt voor jullie deelname!

De NHS-NRG-groep en dan in het bijzonder, Amika, Andrea, Astrid, GertJan, Stef en Tommy, bedankt voor de leuke samenwerking de afgelopen 4,5 jaar en de gezellige halfjaarlijkse etentjes. De Bobwams waren altijd erg productief en een goede uitlaatklep voor de perikelen van een 'natte' AIO.

Ik heb altijd erg genoten van mijn tijd bij $\mathrm{HB}$ en hier hebben een hoop mensen aan bijgedragen. Andrea, Ariënne, Freddy, Guy, Joost, Kristel, Mandy, Manuela, Marieke, Mirjam, Nathalie, Neeltje, Ralph, Stefan en Tanja heel erg bedankt voor de gezellige tijd! Larisa, Claudia, Ilona en Loek jullie tevens heel erg bedankt voor al jullie ondersteunende hulp. Verder wil ik ook de rest van HB bedanken voor de goede werksfeer. Fortunately, I have been very lucky with my new colleagues, Emma, Patrick, Maria and Jonatan thank you for making me feel welcome at Prevnut! Manuela en Ellen, ik ben erg trots dat jullie als paranimfen achter mij zullen staan tijdens de verdediging. Jullie betekenen heel veel voor mij, bedankt voor de mooie vriendschap!

Mijn verhuizing naar Stockholm heeft voor mij nog eens goed benadrukt dat er meer in het leven van een AIO is dan alleen maar hard werken. Merel, Neeltje en Marloes ook al maakten jullie pas later deel uit van mijn Maastrichtse leventje, ik heb erg genoten van onze gezellige avonden en ben blij dat ik jullie mijn vriendinnen mag noemen. Maartje, wat was het weer als van ouds toen jij terug naar Maastricht verhuisden, bedankt voor gezelligheid. De weekendjes Rotterdam, Amsterdam, Den Bosch, Nijmegen, Breda en Utrecht deden mij altijd weer relativeren wat echt belangrijk is in het leven en deden mij vol energie weer terugkeren naar Maastricht. Anna-Rixt, Anke, Annoek, Ingrid, Jojanne, Emely, Ellen, Els, Neeltje, Nienke heel erg bedankt voor alle bijzondere momenten, ik hoop dat er nog velen zullen volgen!

Mam en Pap bedankt dat jullie altijd voor me klaar staan! Ik ben blij dat jullie mij hebben gestimuleerd om mijn eigen beslissingen te nemen en de wijsheid hebben bijgebracht dat er meer wegen naar Rome leiden. Ruben en Hanna door 
jullie hulp en jouw prachtige gedicht Ruben, is dit proefschrift echt een familie werk geworden. Bedankt!

At last, but definately not least Tomas. You really bring the best out in me! Thank you for your enormous postive energy and your dreams, one of which will be becoming reality soon. Looking forward to all to come with you! 
CURRICULUM VITAE 


\section{ABOUT THE AUTHOR}

Lydia Nadine Kwak was born on July 30, 1977 in Amsterdam, the Netherlands. In 1996 she received her VWO diploma at the Rijnlands Lyceum in Oegstgeest. In the same year, she started her study Health Sciences at Maastricht University specializing in Biological Health Sciences and Health Education and Promotion. She did her Master thesis entitled 'How is your walking group running?' at the National Heart Foundation in Brisbane, Australia. In August 2002 she started her PhD research at the department of Human Biology, Maastricht University, where she also finished her second Master thesis. The research performed during her PhD period is described in this thesis. Since April 2007, she is working at the Department of Preventive Nutrition of the Karolinska Institute in Stockholm, Sweden. Where she is currently working on ALPHA, a new European project aimed at physical activity assessment. 


$$
\begin{aligned}
& \text { LIST OF } \\
& \text { PUBLICATIONS }
\end{aligned}
$$




\section{Full papers}

- Kwak, L., Kremers, S.P.J., Walsh, A., \& Brug, J. (2006). How is your walking group running? Health Education, 106, 21-31.

- Kwak, L., Kremers, S.P.J., Van Baak, M.A., \& Brug, J. (2006). Participation rates in worksite-based intervention studies: health promotion context as a crucial quality criterion. Health Education International, 21, 66-9.

- Kwak, L., Kremers, S.P.J., Van Baak, M.A., \& Brug, J. (2007). Formation of implementation intentions promotes stair use. American Journal of Preventive Medicine, 32, 254-5.

- Kwak, L., Kremers, S.P.J., Werkman, A. Visscher, T.L.S., Van Baak, M.A., \& Brug, J. (2007). The NHF-NRG In Balance-project: the application of Intervention Mapping in the development, implementation and evaluation of weight gain prevention at the worksite. Obesity Reviews, 8, 347-61.

- Kwak, L., Kremers, S.P.J., Van Baak, M.A., \& Brug, J. (2007). A poster-based intervention to promote stair use in blue-and white-collar worksites. Preventive Medicine, 45, 177181.

- Kwak, L., Kremers, S.P.J., Brug, J. \& Van Baak, M.A. (in press). Measuring physical activity in field studies: comparison of a questionnaire, $24 \mathrm{~h}$ recall and accelerometer. The European Journal of Sport Science.

- Kwak, L., Kremers S.P.J., Van Baak, M.A. \& Brug, J. (resubmitted, minor revisions). Long-term effectiveness of implementation intentions regarding stair use: a 12 months follow-up.

- Kwak, L., Kremers S.P.J., Candel, M.J.J.M, Visscher, T.L.S., Brug, J. \& Van Baak, M.A. (under review). The effectiveness of the NHF-NRG In Balance-project: short-and longterm changes in sum of skinfolds and waist circumference.

- Kwak, L., Kremers, S.P.J., Visscher, T.L.S., Van Baak, M.A. \& Brug, J. (under review). Behavioural and cognitive effects of a worksite-based weight gain prevention programme: The NHF-NRG In Balance-project.

- Werkman, A., Schuit, A.J., Kwak, L., Kremers, S.P.J., Visscher, T.L.S., Kok, F.J., \& Schouten, E.G. (2006). Study protocol of a cluster randomised controlled trial investigating the effectiveness of a tailored energy balance programme for recent retirees. BMC Public Health, 6, 293.

\section{Abstracts}

- Kwak, L., Van Baak, M.A., Kremers, S.P.J., \& Brug, J. (2003, May). Energy balance in young adults. International Journal of Obesity and related metabolic disorders 2003; 27(1): s135. Poster session presented at the 12th European Congress on Obesity, Helsinki, Finland.

- Kwak, L., Kremers, S., Walsh, A., \& Brug, J. (2003, May). How is your walking group running? International Journal of Obesity and related metabolic disorders 2003; 27(1): s152. Poster session presented at the 12th European Congress on Obesity, Helsinki, Finland.

- Werkman, A., Kwak, L., \& Singh, A. (2004). NHS-NRG interventies: preventie van gewichtsstijging bij scholieren, jongvolwassenen en pas gepensioneerden. Tijdschrift voor Gezondheidswetenschappen.

- Kwak, L., Kremers, S.P.J., Brug, J., \& Van Baak, M.A. (2005, April). Comparison of physical activity assessed through two physical activity questionnaires and monitored by an accelerometer. Paper presented at the NASO Spring Meeting, Maastricht, the Netherlands.

- Kwak, L., Kremers, S.P.J., Brug, J., \& Van Baak, M.A. (2005, June). Comparison of physical activity assessed through two physical activity questionnaires and monitored by an accelerometer. Poster session presented at the European Congress on Obesity, Athens, Greece. 
- Kremers, S.P.J., Visscher, T.L.S., Nooyens, A.C.J., De Bruijn, G.J., Singh, A., Kwak, L., \& Werkman, A. (2005, June). Netherlands research programme weight gain prevention (NHF-NRG): rationale and objectives. Paper presented at the Fourth Annual Conference of the International Society of Behavioural Nutrition and Physical Activity, Amsterdam, the Netherlands.

- Kwak, L., Singh, A., Werkman, A., Kremers, S.P.J., \& Visscher, T.L.S. (2005, June). NHFNRG interventions: prevention of weight gain in adolescents, young adults and recently retired. Netherlands research programme weight gain prevention (NHFNRG): rationale and objectives. Paper presented at the Fourth Annual Conference of the International Society of Behavioural Nutrition and Physical Activity, Amsterdam, the Netherlands.

- Kwak, L., Kremers, S.P.J., Van Baak, M.A., \& Brug, J. (2005, October). NHF-NRG interventions: prevention of weight gain in young adults. Poster session presented at the NASO Prevention Symposium, Utrecht, the Netherlands.

- Kwak, L., Kremers, S.P.J., Van Baak, M.A., \& Brug, J. (2006, July). Prompts and implementation intentions promote stair use in worksite settings. Paper presented at the Fifth Conference of the International Society of Behavioral Nutrition and Physical Activity, Boston, United States of America.

- Kwak, L., Kremers, S.P.J., Brug, J., \& Van Baak, M.A. (2006, November). NHF-NRG In Balance-project: prevention of weight gain in young adults. Paper presented at the NASO conference, Doorwerth, the Netherlands.

- Kwak, L., Kremers, S.P.J., Van Baak, M.A., \& Brug, J. (2007, August). The use of short interventions in promoting stair use in worksite settings. Paper presented at the Twenty-first European Health Psychology Society, Maastricht, the Netherlands. 

